University of Louisville

ThinkIR: The University of Louisville's Institutional Repository

Electronic Theses and Dissertations

$12-2019$

\title{
Non-invasive detection and assessment of coronary stenosis from blood mean residence times.
}

Javad Hashemi

University of Louisville

Follow this and additional works at: https://ir.library.louisville.edu/etd

Part of the Complex Fluids Commons, Other Chemical Engineering Commons, and the Transport Phenomena Commons

\section{Recommended Citation}

Hashemi, Javad, "Non-invasive detection and assessment of coronary stenosis from blood mean residence times." (2019). Electronic Theses and Dissertations. Paper 3305.

https://doi.org/10.18297/etd/3305

This Doctoral Dissertation is brought to you for free and open access by ThinkIR: The University of Louisville's Institutional Repository. It has been accepted for inclusion in Electronic Theses and Dissertations by an authorized administrator of ThinkIR: The University of Louisville's Institutional Repository. This title appears here courtesy of the author, who has retained all other copyrights. For more information, please contact thinkir@louisville.edu. 
NON-INVASIVE DETECTION AND ASSESSMENT OF CORONARY STENOSIS

FROM BLOOD MEAN RESIDENCE TIMES

by

Javad Hashemi

B.Sc., University of Sistan and Baluchestan, 2010

M.Sc., Tarbiat Modares University, 2013

\author{
A Dissertation \\ Submitted to the Faculty of the \\ University of Louisville \\ J. B. Speed School of Engineering \\ as Partial Fulfillment of the Requirements \\ for the Degree \\ Doctor of Philosophy \\ in Chemical Engineering \\ Department of Chemical Engineering \\ University of Louisville \\ Louisville, Kentucky
}

December 2019 

NON-INVASIVE DETECTION AND ASSESSMENT OF CORONARY STENOSIS

FROM BLOOD MEAN RESIDENCE TIMES

$$
\text { By }
$$

Javad Hashemi

B.Sc., University of Sistan and Baluchestan, 2010

M.Sc., Tarbiat Modares University, 2013

\section{A Dissertation Approved on}

December 2, 2019

By the following Dissertation Committee

R. Eric Berson, (Dissertation Director)

Shahab Ghafghazi

Xiao-An Fu

Vance W. Jaeger

Joseph B. Moore IV 


\section{DEDICATION}

This dissertation is devoted to my parents

Mr. Ali Hashemi

Mrs. Roghayeh Hosseini

whose love and support for me have been immeasurable. 


\section{ACKNOWLEDGMENTS}

I would like to thank my advisors, Prof. R. Eric Berson and Dr. Shahab Ghafghazi for their guidance and patience. I would also like to thank the other committee members, Dr. XiaoAn Fu, Dr. Vance W. Jaeger and Dr. Joseph B. Moore IV, for their willingness to serve on my dissertation committee. I would also like to thank my parents, Ali Hashemi and Roghayeh Hosseini, as well as my brother, Reza and Sisters, Zohreh, Mansooreh and Firoozeh, for their support and encouragement. 


\section{ABSTRACT \\ NON-INVASIVE DETECTION AND ASSESSMENT OF CORONARY STENOSIS FROM BLOOD MEAN RESIDENCE TIMES}

Javad Hashemi

December 2, 2019

Coronary artery stenosis is an abnormal narrowing of a coronary artery caused by an atherosclerotic lesion that reduces lumen space. Fractional flow reserve (FFR) is the gold standard method to determine the severity of coronary stenosis based on the determination of rest and hyperemic pressure fields, but requires an invasive medical procedure. Normal FFR is 1.0 and FFR $\leq 0.80$ is considered hemodynamically significant. Computational fluid dynamics (CFD) enables computation of noninvasive FFR in conjunction with patient specific imaging such as angiography or computerized tomography coronary angiography, but has typically resulted in $8-12 \%$ false readings. An alternate CFD method is presented here that computes blood residence time through a given coronary artery segment, which provides a more direct assessment of physiological changes as a result of the stenosis. Small regions of recirculatory flow form distal to a stenosis causing an increase in the mean residence time of the blood relative to the nominal mean residence time, defined as volume divided by flow rate. Blood mean residence time was expressed as a dimensionless parameter, Blood $\mathrm{RT}_{\mathrm{R}}$, to account for varying volume and flow rate of individual segments. Blood ${ }_{\mathrm{RT}}$ was computed in 100 patients who had undergone the pressure-wire FFR procedure, and a threshold for Blood RT was 
determined to assess the physiological significance of a stenosis, analogous to the diagnostic threshold for FFR. The threshold exhibited excellent discrimination in detecting significant from non-significant stenosis compared to the gold standard pressure-wire FFR, with sensitivity of $98 \%$ and specificity of $96 \%$. When applied to clinical practice, this could potentially allow practicing cardiologists to accurately assess and quantify the severity of coronary stenosis without resorting to invasive catheter-based techniques.

The first 100 patient study required a clinically determined blood flow rate as a key model input. To create a more non-invasive process, a multiple linear regression approach was employed to determine blood flow rate entering a given artery segment. To validate this method, Blood $_{\mathrm{RT}}$ was computed for a new set of 100 patients using the regression derived blood flow rate. The sensitivity and specificity were $95 \%$ and $97 \%$, respectively, indicating similar discrimination compared to the clinically derived flow rate.

The method was also applied to a succession of stenosis in series. When Blood $\mathrm{RT}_{\text {of }}$ each individual stenosis was well above the threshold for significance, the cumulative effect of all stenoses led to an overall Blood $\mathrm{RT}_{\mathrm{T}}$ below the threshold of hemodynamic significance. 
TABLE OF CONTENTS

PAGE

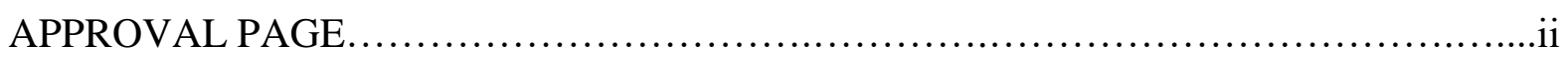

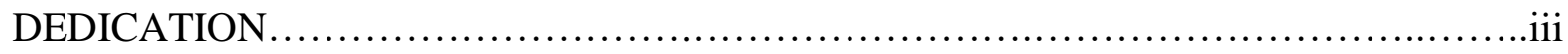

ACKNOWLEDGMENTS .................................................................

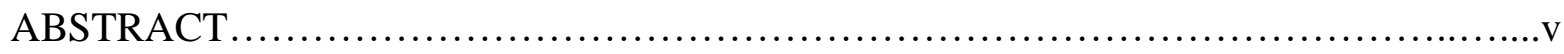

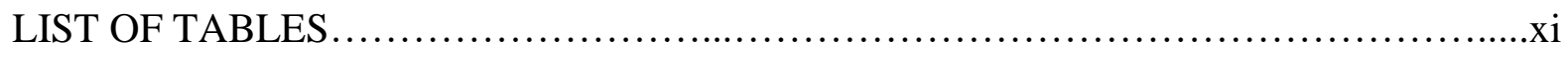

LIST OF FIGURES......................................................................

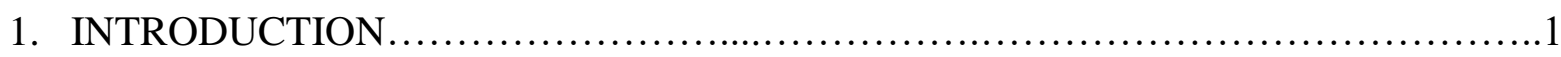

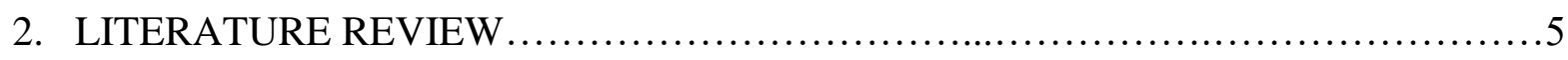

2.1 CFD Method ...................................................................... 5

2.2 CFD for Hemodynamics.............................................................

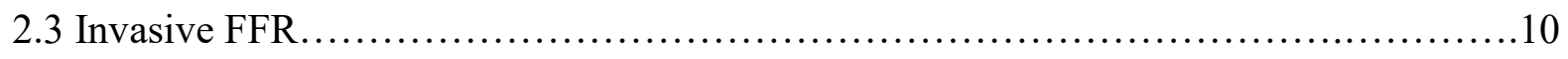

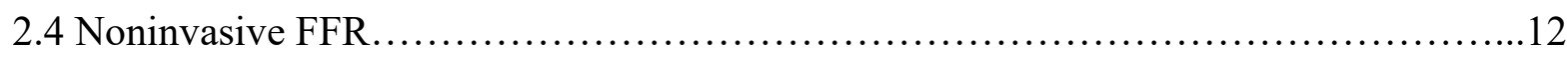

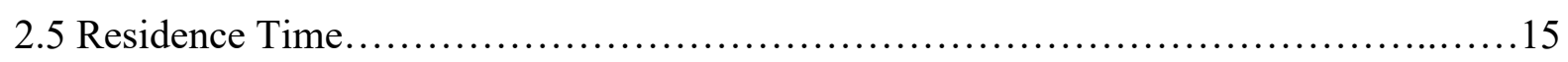

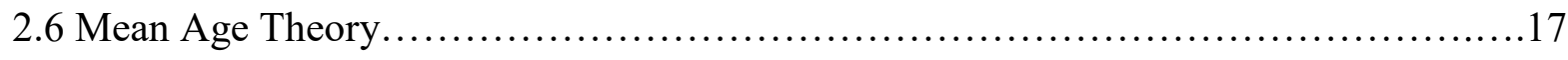


2.6 Residence Time Applications in Cardiovascular Research....

3. EXPERIMENTAL METHODS ...............................................25

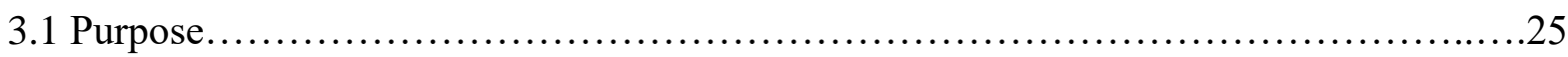

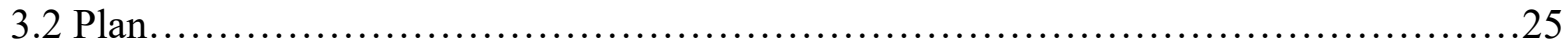

3.3 First Aim. Blood Residence Time to Assess Significance of Coronary Artery

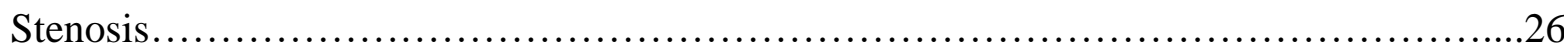

3.3.1 Patient Population........................................................... 26

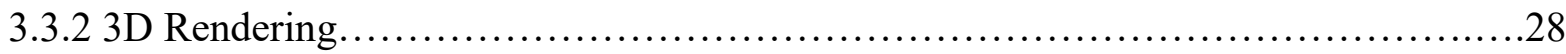

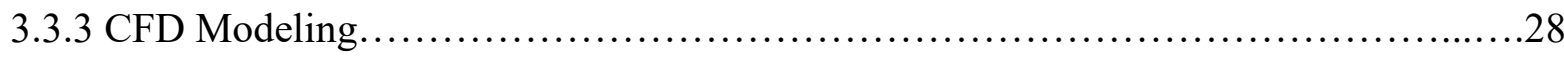

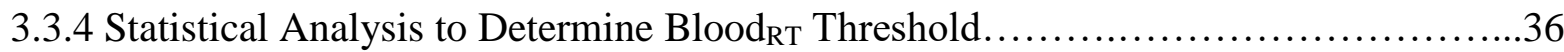

3.4 Second Aim. Assessment of Significant Stenosis for Coronary Artery with Serial

Stenoses................................................................... 36

3.4.1 3D Rendering and Patients' Arteries........................................ 36

3.4.2 CFD Modeling ........................................................ 38

3.5 Third Aim. Volume Flow Rate Determination Using Machine Learning................39

3.5.1 Regression Model to Determine Blood Flow Rate................................39

4. BLOOD RESIDENCE TIME TO ASSESS SIGNIFICANCE OF CORONARY ARTERY

STENOSIS .................................................................... 41 
4.1 Pathlines

4.2 Wall Shear Stress................................................................ 43

4.3 Mean Residence Time.......................................................44

4.4 Blood $_{\mathrm{RT}}$ and Threshold.........................................................

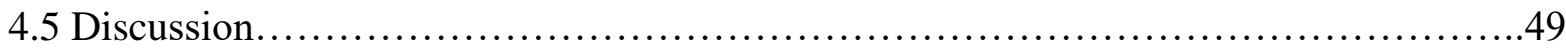

5. ASSESSMENT OF SIGNIFICANT STENOSIS FOR CORONARY ARTERY WITH

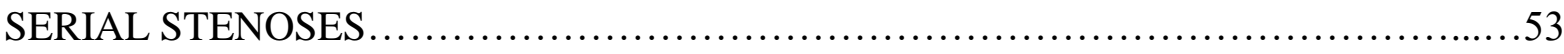

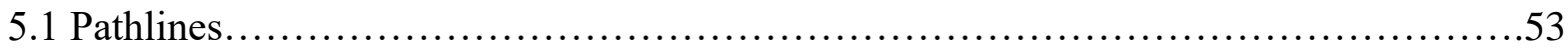

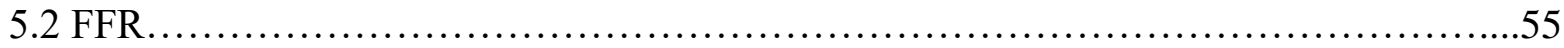

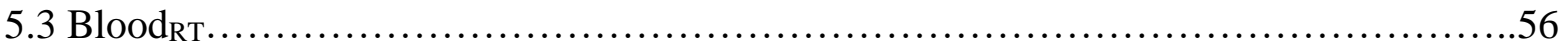

5.4 Wall Shear Stresses..............................................................

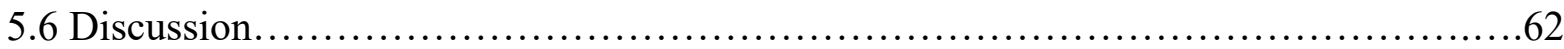

6. Volume Flow Rate Determination Using Multiple Linear Regression..................64

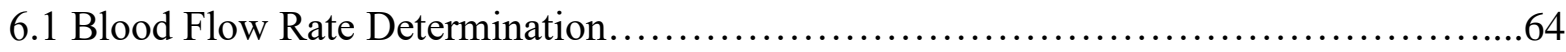

6.2 Blood $_{\mathrm{RT}}$ Computed with Predicted Coronary Blood Flow Rate........................69

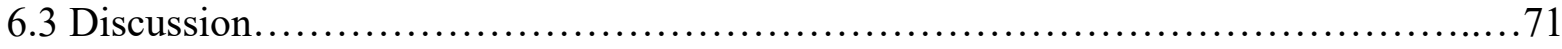

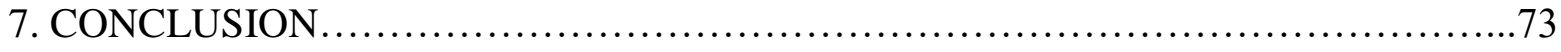

8. RECOMMENDATIONS ................................................... 75

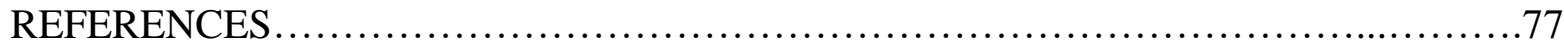




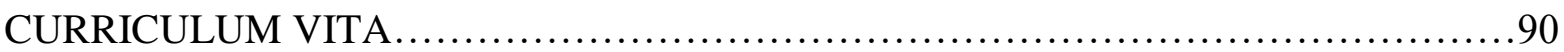




\section{LIST OF TABLES}

PAGE

Table 3.1 Clinical characteristics of patients.......................................27

Table 3.2 Characteristics of patients enrolled in the study............................ 37

Table 4.1 Statistical analysis comparison between Blood $_{\mathrm{RT}}$ and various forms of virtual

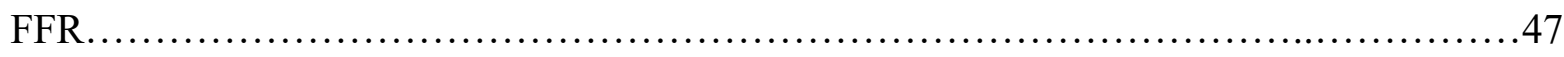

Table 5.1 Local and Total Blood $\mathrm{RT}_{\mathrm{T}}$ and vFFR for each patient and stenosis..................59

Table 6.1 Regression model for blood flow rate clustered by segment type..................65 


\section{LIST OF FIGURES}

PAGE

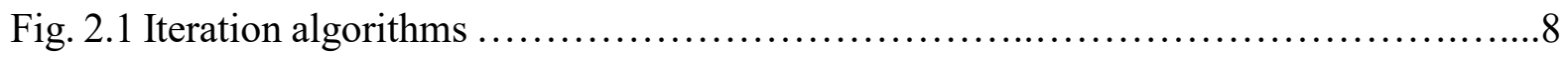

Figure 2.2 (a) RTD for near plug flow reactor and (b) RTD for near perfectly mixed

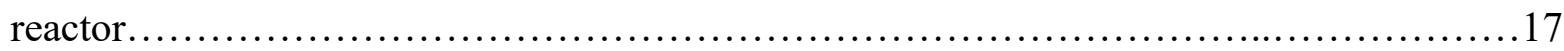

Figure 3.1 3D rendering steps and diameter and area results............................28

Figure 3.2. Mesh generation for a coronary artery, inlet, and outlet.......................29

Figure 3.3 Hyperemic boundary conditions.............................................

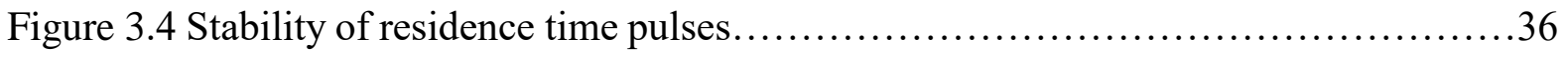

Figure 3.5 3D-rendering patient's coronary arteries from angiography $\ldots \ldots \ldots \ldots \ldots \ldots \ldots \ldots . \ldots \ldots$

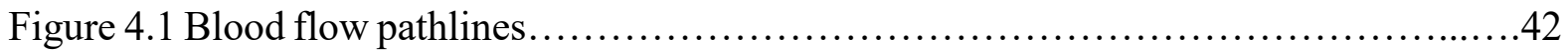

Figure 4.2 Wall shear stress $(\mathrm{Pa})$ in two sample patients................................43

Figure 4.3 Mean residence time (s) pathlines...........................................45

Figure 4.4 Mean residence time throughout one cardiac pulse............................46

Figure 4.5 (a) Blood ${ }_{\mathrm{RT}}$ and invasive FFR for 100 patient coronary arteries and (b) Receiver operator characteristic (ROC) curve analysis .......................................49

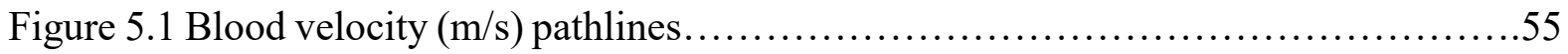


Figure 5.2 Localized FFR wall contours.

Figure 5.3 Slices of blood residence time (s) contours and pathlines.....................58

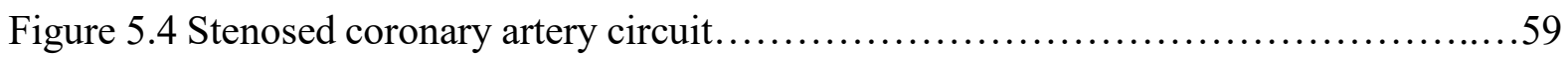

Figure 5.5 Blood WSS $(\mathrm{Pa})$ contours on the wall...................................61

Figure 6.1 Predicted and clinical-based volume flow rate $\ldots \ldots \ldots \ldots \ldots \ldots \ldots \ldots \ldots \ldots . \ldots 6$

Figure 6.2 (a) Blood $_{\mathrm{RT}}$ and invasive FFR for 100 patient coronary arteries and (b) ROC

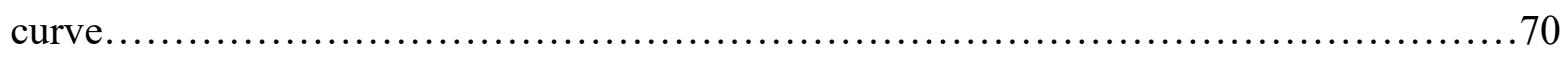




\section{CHAPTER ONE}

\section{INTRODUCTION}

One million invasive coronary angiographies (ICAs) are performed on a yearly basis in the US and approximately half of these patients undergo percutaneous coronary intervention (PCI) and stent placement [1]. In patients for whom the indication for ICA is stable coronary artery disease (CAD) or chest pain, frequently, the interventional cardiologist encounters coronary stenosis of 40 to $90 \%$ and a decision has to be made with regards to revascularization (stent placement). The prevailing clinical practice is to visually estimate the degree of coronary blockages; patients with a stenosis $\geq 60$ to $70 \%$ would then undergo PCI while others would receive medical therapy despite the fact that the totality of randomized trial data to date has not shown an improvement in patients' outcomes when revascularization is guided by degree of stenosis alone [2, 3]. Although, there is a fairly consistent relationship between the degree of coronary stenosis and coronary blood flow, the relation between the two is complex and there remains a disconnect between them in a significant number of cases [4] .

Accurate quantification of coronary stenosis is crucial in order to provide optimal medical care for patients. FFR, measured by placing a pressure wire across a stenotic lesion, is the gold standard method to determine the severity of coronary stenosis but requires an invasive medical procedure [5]. FFR is the ratio of maximal blood flow distal to a stenotic lesion to maximal flow in the same artery if hypothetically normal [6]. Normal FFR is 1 and an FFR $\leq 0.80$ is considered hemodynamically significant [7]. The challenge is that 
measurement of FFR is an invasive extra step that is expensive, time consuming and associated with higher risk of complications, hence the slow acceptance rate of the technology by interventional cardiologists.

FFR has also been used for patients with serial stenoses in the same coronary artery [8]. A sensor pressure wire is pulled-back through all present stenoses to estimate which of the stenoses has a significant effect on the overall hemodynamics and the largest pressure gradient [8]. The pull-back method is repeated so as to accurately find the functionally significant stenosis among the other remaining stenoses. Assessing significant stenosis or stenoses under maximal hyperemia is important for deciding the proper location for stent placement, as stenting the primary stenosis can improve blood flow even with other stenoses present in the coronary artery [9]. Previous studies proved that the ratio of distal to proximal pressure would not accurately assess the FFR of each individual lesion if the other lesions were ignored [9]. The individual effect of each stenosis would be unpredictable using the simple ratio of the distal and proximal pressures [8]. CT-FFR was attempted for serial stenosis, but was not accurate to assess significant stenosis in distal coronary artery segments [10].

Recent advances in CFD enable calculation of coronary flow and pressure fields from anatomic image data [11]. Noninvasive calculation of FFR, or virtual FFR (vFFR), has been performed on images obtained from ICA or CTCA $[12,13]$. This method applies CFD to determine the physiologic significance of CAD without additional imaging, administration of medications, or use of pressure-wire and hyperemia.

The volume flow rate of blood proximal to a coronary stenosis would be an extremely helpful inlet boundary condition, if known, to enable computation of vFFR without the need to model blood flow throughout the entire coronary tree. One solution to this problem is the 
use of a fixed volume flow rate in all patients [14]. However, there is a significant variation in volume flow rate from patient to patient and it is mostly dependent on the location of stenosis. Other attempts to determine individualized flow rates proved to be quite time consuming and requires invasive techniques.

Residence time is an indicator of flow trajectory and mixing. Deceleration of blood flow during the cardiac cycle yields large recirculation zones distal to stenosis leading to protracted path lengths and greater residence times. CFD is ideal for computing residence time in flow systems. Rather than measuring or computing pressure loss to quantify physiological significance, a new approach is presented here which directly quantifies the altered flow trajectories through a dimensionless form of residence time, $\operatorname{Blood}_{\mathrm{RT}}$, a metric that quantifies variations in time spent in a given stenotic artery segment due to variations in paths through the segment.

Specific Objectives:

1. Determine a threshold for Blood $_{\mathrm{RT}}$ that demonstrates discrimination in the diagnosis of significant from non-significant stenosis, analogous to the diagnostic threshold for FFR. Maximize the sensitivity and specificity of this metric compared with the gold standard pressure wire FFR threshold for 100 patients.

2. Determine the individual physiological significance of each stenosis in a system of serial stenoses.

3. Determine the flow rate of blood using multiple linear regression. This first requires determining the statistically significant readily available patient factors that affect the value. The coronary volume flow rate is an essential 
model inlet boundary condition for determining $\operatorname{Blood}_{R T}$ (or vFFR), and is not available through non-invasive means.

4. Validate the model for calculation of Blood $\mathrm{RT}_{\mathrm{T}}$ using the regression derived flow rates in a second cohort of 100 patients against FFR. 


\section{CHAPTER TWO \\ LITERATURE REVIEW}

\subsection{CFD Method}

CFD is a method to study fluid dynamics by applying numerical methods and data processing techniques for solving fluid flow problems. CFD is based on transport phenomena that includes the conservations of mass, momentum, and energy. CFD programs improve the ability to solve these otherwise impractical conservation equations effectively and simultaneously. Conservation equations could be solved using a discretized version of a geometric domain. ANSYS programs such as Fluent and CFX can simulate fluid flow and calculate relevant fluid user-defined metrics. In general, these programs permit the simulation of flow, heat transfer, and reactions for industrial applications and, as relevant here ,biomedical applications. Bioengineers have extended CFD to investigate complex physiological flows especially in cardiovascular medicine, and can enhance diagnostic assessment, device design, and clinical trials.

CFD solves governing conservation equations that depend on the individual system. The equation for energy conservation can be included in addition to the conservation equations of mass and momentum. Continuity is described for the conservation equation of mass by:

$$
\frac{\partial \rho}{\partial t}+(\nabla \cdot \rho \vec{v})=S_{m}
$$

where $\rho$ is density, $v$ is velocity, and $S_{m}$ is a source term. The momentum conservation equation is: 


$$
\frac{\partial}{\partial t}(\rho \vec{v})+\nabla \cdot \rho \vec{v} \vec{v}=-\nabla p-[\nabla \cdot \tau]+\rho g \vec{~}
$$

The viscous momentum flux tensor $\boldsymbol{\tau}$ represents shear stress and is given by:

$$
\tau=-\mu\left[\nabla \vec{v}+(\nabla \vec{v})^{T}\right]+\frac{2}{3} \mu(\nabla \cdot \vec{v}) \delta
$$

Assuming constant viscosity and fluid density, substitution of $\boldsymbol{\tau}$ in the conservation equation of momentum and adjustment gives the Navier-Stokes equation:

$$
\rho\left(\frac{\partial \vec{v}}{\partial t}+\vec{v} \cdot \nabla \vec{v}\right)=-\nabla p+\mu \nabla 2 \vec{v}+\rho g \vec{~}
$$

CFD solves the mass conservation and Navier-Stokes equations by approximating the continuous variables in the partial differentials using discrete analogs and utilizing a geometric mesh or grid discretized domain. For CFD, flow variables are defined at every point on the domain for continuous domains. An accurate solution may be obtained when each node interacts with adjacent nodes predictably. Discretized results approach analytical results when the grid resolution (i.e., mesh count) is sufficient. The fundamental ideas of CFD are illustrated by employing them in the following simple $1 \mathrm{D}$ equation [15]:

$$
\frac{d u}{d x}+u=0 ; 0 \leq x \leq 1 ; u(0)=1
$$

The domain could be discretized into equally-spaced $(\Delta x)$ points on a grid, with general grid points labeled $x_{i}$. Since the governing equation is valid at any grid point, this gives:

$$
\left(\frac{d u}{d x}\right)_{i}+u_{i}=0
$$

where the subscript $i$ represents the value at grid point $x_{i}$. For getting an expression for differential term in terms of $u$ at the grid points, $\mathrm{u}_{\mathrm{i}}-1$ is expanded in a Taylor's series:

$$
u_{i-1}=u_{i}-\Delta x\left(\frac{d u}{d x}\right)_{i}+O(\Delta x)
$$

and then: 


$$
\left(\frac{d u}{d x}\right)_{i}=\frac{u_{i}-u_{i-1}}{\Delta x}+O(\Delta x)
$$

where $\mathrm{O}(\Delta \mathrm{x})$ is the truncation error, without higher-order terms in the Taylor series, this equation is reached:

$$
\frac{u_{i}-u_{i-1}}{\Delta x}+u_{i}=0
$$

Fluent programing uses the finite-volume method. Integral forms of the conservation equations are applied to the finite-volume method. A cell of control volume is assigned discrete equations for each cell. In the finite-volume method, a control volume is usually referred to as a "cell" and a grid point as a "node". In 2D, mesh could have three or four sided cells. In 3D, cells are usually hexahedrals, tetrahedrals, or prisms. The integral form of the conservation equation of mass in this method is:

$$
\int_{S}(\vec{v} \cdot \hat{n}) d S=0
$$

For a rectangular cell with length $\Delta x$, height $\Delta y$, and applying a velocity vector

of $\vec{V}_{i}=u_{i} \hat{\imath}+v_{i} \hat{\jmath}$, the integral equation in discretized form is:

$$
-u_{1} \Delta y-v_{2} \Delta x+u_{3} \Delta y+v_{4} \Delta x=0
$$

The above equation is the continuity equation in discrete form calculated in each cell. Other conservation equations can be discretized for each cell in the same way. In addition, the finite volume method could be extendable to different cell shapes in two or three dimensions.

After discretization, equations are solved simultaneously and iteratively. Fluent has two options for iterating. These are pressure-based and density-based solvers (Figure 2.1). The iteration number is important to reach a convergence with good resolution results. The results of each iteration are compared with the previous iteration, and when residual values of the two iterations are sufficiently small, then the results have converged sufficiently. 


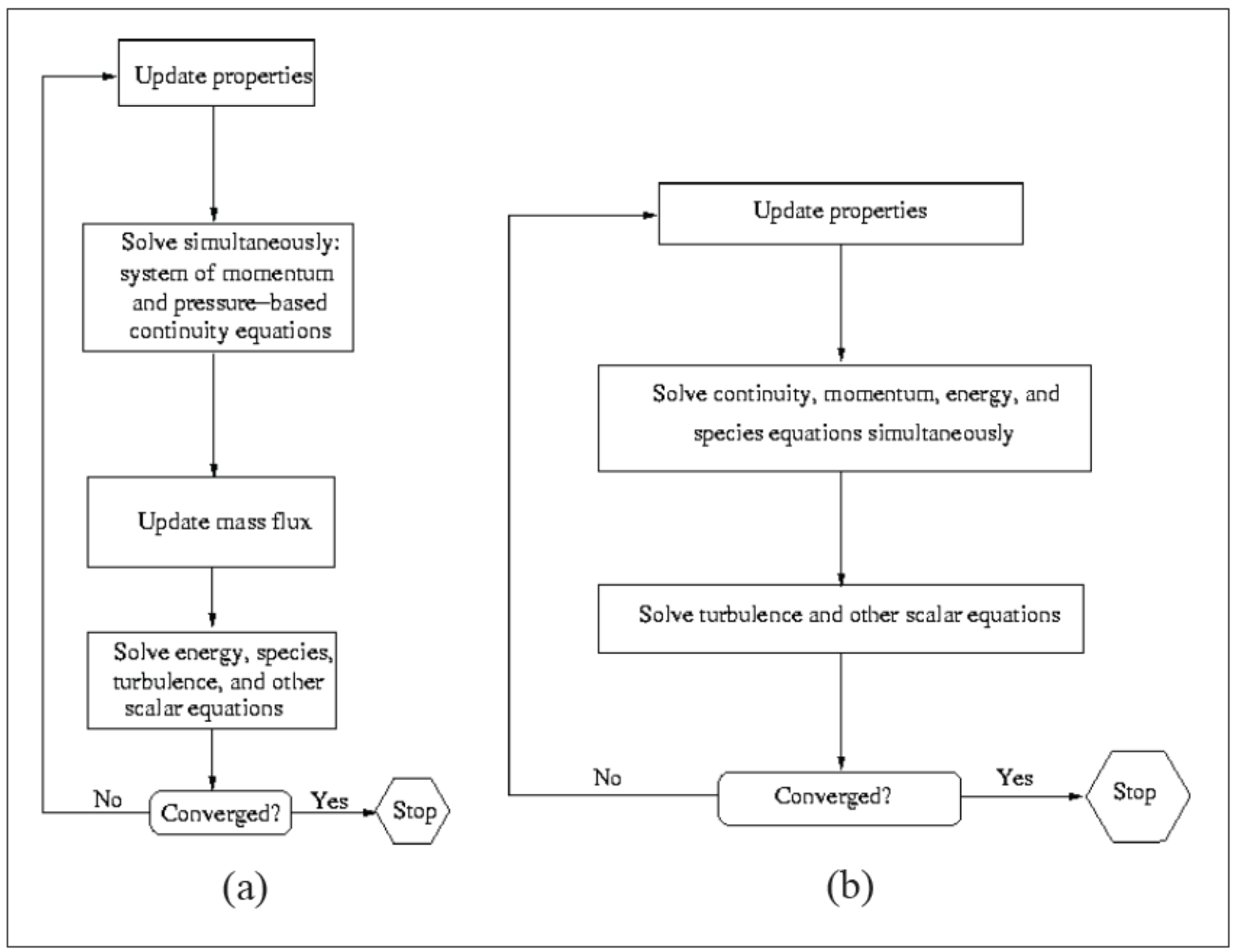

Fig. 2.1 Iteration algorithms for the (a) Pressure-based and (b) Density-based Solvers [16].

\subsection{CFD for Hemodynamics}

Hemodynamic analysis plays an important role for studying and diagnosing cardiovascular disease. Steinman et al. [17] showed that studying different biomechanical factors in the cardiovascular system could be helpful in understanding arterial disease, including atherogenesis, and in the diagnosis and treatment of patients with cardiovascular disease. Qiu and Tarbell [18] predicted low wall shear stress (WSS) on the inside curvature of an idealized model of a left coronary artery using their single-phase computational fluid dynamics (CFD) model. Zeng et al. [19] attempted but could not find a good correlation 
between their single-phase WSS using CFD simulations and curvature in a patient specific reconstruction of a right coronary artery. Steinman [20] demonstrated that the last three decades of studies had a lack of progress because most studies have been retrospective using idealized or averaged hemodynamic models rather than being prospective using local measured hemodynamic indices and correlated in a noninvasive manner using CFD. Migliavacca et al. [21] computed three-dimensional pulsatile coronary flow and pressure in a single coronary artery with an idealized model and low mesh resolution.

Steinman [20] presented the governing equations and constitutive relations of singlephase cardiovascular modeling. Gidaspow [22] reviewed and presented 30 years of study on multiphase theory and CFD modeling. The rheological model for blood viscosity is a factor in the simulation of hemodynamic flows [23]. However, non-Newtonian models have been developed for modeling [23], but developing the appropriate non-Newtonian models for complex flow is still a challenge. Some investigators modeled blood as multiphase fluid, and they used a non-Newtonian hemodynamic model for the secondary-phase of blood, and the continuous plasma phase was assumed a Newtonian fluid [24]. Bird et al., [25] extended the Carreau-Yasuda viscosity model or using mixture viscosity, and they found a good correlation between the model with experimental data. Recently, some researchers showed that Newtonian viscosity can be sufficient for single phase model and is better than available non-Newtonian models $[26,27]$. The non-Newtonian properties are considered as a secondary effect while reconstructing accurate anatomic data and boundary conditions are more important factors [28].

Generally, arterial flow is laminar even in curves and branches [29]. Furthermore, most investigators have assumed laminar flow in stenosed arteries [30, 31]. Ku et al. [32] showed 
that $80 \%$ of the pressure loss $(\mathrm{FFR}=0.2)$ is required before, severe turbulence is observed although such degree of stenosis is universally treated with coronary intervention in clinical practice.

\subsection{Invasive FFR}

The degree of stenosis is usually estimated visually, which is notoriously inaccurate [33]. The gold standard of care for quantification of coronary lesions is invasive measurement of FFR [5]. During coronary angiography, FFR can be measured using a pressure wire, which calculates the ratio of the distal coronary artery pressure to the proximal pressure when adenosine is used to induce maximal hyperemia. FFR is the ratio of maximal blood flow distal to a stenotic lesion to maximal flow in the same artery if hypothetically normal [6]. Normal FFR is 1 and an FFR $\leq 0.80$ is considered hemodynamically significant [7]. The challenge is that measurement of FFR is an invasive extra step that is time consuming, expensive, and associated with additional risk.

In hearts with epicardial stenosis, Uren et al. [34] demonstrated that increasing coronary stenosis above $40 \%$ would potentially influence blood flow in hyperemic condition and limit maximal blood flow, therefore, physiologically significant. Gould et al. [35] developed a physiologic measure of coronary stenosis firstly, and later developed the relative coronary flow reserve concept [36], defined as maximal flow with a stenosis divided by normal maximal flow without the stenosis. Gould's method was needed on direct flow measurement. Pijls et al. [37] developed and presented invasive FFR encompassing its different aspects from the effect of angiographic ambiguity in assessment of coronary stenosis, clinical hyperemic drugs and their use in determination of invasive FFR, miniature pressure sensors, and a theoretic framework for interpreting the ratio of distal coronary to driving aortic pressure. This 
method overcomes some of the technical difficulties encountered with direct flow measurement pioneered by Gould [36]. Fractional flow reserve is defined as the maximal myocardial blood flow in the presence of a stenosis, divided by the theoretical maximal flow in the absence of the stenosis in the same artery $[37,38]$. The fraction of normal maximal blood flow that could be reached to myocardium area due to a given stenosis is represented by FFR. FFR is developed based on a Poiseuille's Law modification [38] which states that in a fluid system, the flow (Q) equals the pressure difference across the system (DP) divided by the resistance (R). To measure FFR, intravenous adenosine is used to minimize the resistance of the collateral circulation and myocardial vascular bed [39]. The assumption is that the coronary lesion of interest is maximally flow limiting, and the effect of the collateral circulation and myocardial vascular bed on pressure drop across the lesion is minimal and constant. Additionally, if the collateral circulation were to make a physiologically important contribution at maximal dilatation, then the pressure drop across the coronary lesion of interest would be smaller as a result of that contribution. Therefore, the FFR will reflect the physiologic significance of a lesion both in presence and absence of significant collateral blood flow. Pijls et al [37] described derivation of fractional flow reserve with some assumptions. The important principle, as described above, is that the flow through a specific portion of the myocardium equals the pressure difference across that region divided by the resistance of the of regional myocardium. For a stenotic artery, then $D P$ is the pressure distal to the stenosis (Pd) minus the venous pressure $(\mathrm{Pv})$. For an artery without a stenosis, the $\Delta \mathrm{P}$ would be the arterial pressure (Pa) minus the venous pressure $(\mathrm{Pv})$. Therefore:

$$
F F R=\frac{P_{d}-P_{v} / R}{P_{a}-P_{v} / R}=\frac{P_{d}-P_{v}}{P_{a}-P_{v}}
$$


If $\mathrm{P}_{\mathrm{v}}$ will be constant, it can be ignored, and FFR can be estimated as $(\mathrm{Pd}) /(\mathrm{Pa})(9)$. In any normal artery the FFR is 1. In 1995 Pijls et al. [38] demonstrated that there is a correlation between inducible ischemia and FFR of lower than 0.74. Later studies demonstrated that a threshold of 0.8 has a better balance of sensitivity and specificity for treatment of ischemic myocardium in patients with obstructive coronary artery disease.

\subsection{Noninvasive FFR}

Advances in CFD allow calculation virtual FFR (vFFR) using high quality coronary CT angiography (CCTA) images ( $\left.\mathrm{FFR}_{\mathrm{CT}}\right)$. CFD quantification of coronary stenosis is based on the Navier-Stokes equations $[11,40]$. To calculate vFFR using CFD, the first step is to reconstruct the coronary artery from CCTA, as well as defining the "boundary conditions" of the coronary artery [41]. FFR $_{\mathrm{CT}}$ screens for important coronary artery disease $(\mathrm{CAD})$ before the patient comes to the cath lab, and then once in the lab, the operators can confirm lesion significance with invasive FFR. Because of the complex fluid and flow characteristics and nonlinear computational model, however, extensive computational power and time are needed. FFR $_{\mathrm{CT}}$ has been correlated with FFR in several studies [42, 43]. The DISCOVER-FLOW (Diagnosis of Ischemia-Causing Stenoses Obtained Via Noninvasive Fractional Flow Reserve) Study was performed to compare FFR $_{\mathrm{CT}}$ and invasive FFR in 103 patients with coronary stenosis of more than 50\% [44]. On a per-vessel basis, the accuracy, sensitivity, specificity, were $84.3 \%, 87.9 \%$, and $82.2 \%$ respectively, for $\mathrm{FFR}_{\mathrm{CT}}$ and $58.5 \%, 91.4 \%$, and $39.6 \%$, respectively, for CCTA alone. The DeFACTO trial investigated the accuracy of FFR $\mathrm{CT}_{\mathrm{C}}$ against invasive FFR [43]. FFR $\mathrm{CT}_{\mathrm{C}}$ accuracy was $73 \%$, with sensitivity and specificity of $90 \%$, and $54 \%$ respectively. The NXT (Analysis of Coronary Blood Flow Using CT Angiography: Next

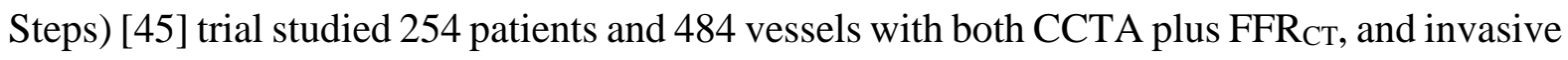


coronary angiography and invasive FFR. Area under curve (AUC) for $\mathrm{FFR}_{\mathrm{CT}}(0.90)$ significantly improved versus standard coronary CTA (0.81). In addition, sensitivity and specificity per-vessel were $86 \%$ and $79 \%$ for FFR $_{\mathrm{CT}}$, vs. $94 \%$ and $34 \%$ for CCTA respectively [45].

Invasive FFR and $\mathrm{FFR}_{\mathrm{CT}}$ have both been studied in patients with serial coronary stenoses [10]. De Bruyne et al. [9] demonstrated for the first time, that in a large animal experimental model, the interaction between two serial stenoses was such that invasive FFR of each lesion separately could not be accurately measured by the equation for isolated stenoses ( $\mathrm{Pd} / \mathrm{Pa}$ during hyperemia) applied to each separately. However, they successfully predicted the FFR of individual lesions by complete equations taking into account coronary pressures before and after every lesion and coronary wedge pressure[8]. In a later study this concept was validated in patients with coronary stenosis. FFR ${ }_{\mathrm{CT}}$ has been compared to FFR pullback curves along the stenoses and pressure recovery rate correlates between FFR $_{\mathrm{CT}}$ and FFR for each stenosis [10]. However, for the same reasons detailed above for invasive FFR, FFR $\mathrm{CT}_{\mathrm{C}}$ is unable to separate the effect of serial stenoses on one another [10]. To resolve this problem, a modified equation has been proposed and validated in FFR ${ }_{\mathrm{CT}}$ [46]. Thus, both invasive FFR and FFR $\mathrm{CT}$ quantification for individual stenosis in coronary arteries with serial stenoses is complicated due to interdependence of such stenoses [46].

vFFR has also been quantified from coronary angiography using computational flow dynamics (CFD). Determining vFFR accurately depends on accuracy of the geometric renderings and model inputs. Empirical resistance boundary conditions at every coronary outlet is typically used [11, 12] but determining accurate values remains a dilemma [47]. Published data reports $6-12 \%$ combined false positive and false negatives for vFFR as 
compared to FFR $[48,49]$. Both FFR and vFFR are a function of pressure loss, a form of energy loss due to friction between fluid and the walls and between layers of the fluid itself. There are additional significant frictional losses around bends and through constrictions. In blood flow through stenotic arteries, recirculation regions are known to form distal to the stenosis [50-52], which also present a major source of frictional and hence pressure loss. Blood is typically modeled as laminar, although localized regions of turbulence can exist in a recirculation region, and not accounting for the turbulent energy dissipation may reduce the accuracy of the predicted pressure loss. Even if modeled as turbulent, the velocity terms are still generally empirical.

Morris et al. were first to compute vFFR from coronary angiography [53]. Thirty-five patients were investigated using vFFR with invasive coronary angiography as the gold standard. They reported accuracy, sensitivity, and specificity of 97\%, 86\%, and 100\%, respectively. Papafaklis et al. [14] introduced virtual functional assessment index (vFAI) that was calculated using the lesion-specific pressure gradient $(\Delta \mathrm{P})$ and constructed the $\Delta \mathrm{P}-\mathrm{flow}$ curve. The model provided high accuracy, sensitivity and specificity of $88 \%, 90 \%$ and $86 \%$, respectively with the optimal cut-point of 0.82 [14]. Another metric introduced, QFR (Quantitative Flow Ratio, Medis Medical Imaging Systems), was examined against invasive FFR and reported an accuracy of $92.4 \%$ on patient-level analysis [54]. In addition, QFR had sensitivity and specificity of $78 \%$ and $89 \%$, respectively [54]. The overall QFR accuracy was 85\%. Pellicano et al. [55] introduced another vFFR model named angiography-derived FFR measurement $\left(\mathrm{FFR}_{\text {angio }}\right)$. A total of 203 lesions in 184 patients were studied. FFR angio had a good correlation $(\mathrm{P}=0.90)$ with the FFR. Optimal cutoff value was 0.80 , with sensitivity, specificity, and accuracy of $88 \%, 95 \%$, and $93 \%$, respectively [55]. 
An important challenge in angiographically calculated vFFR of coronary lesions is that coronary inlet volume flow rate, defined as the volume flow rate of blood proximal to coronary stenosis, is required as an inlet boundary condition to enable computation of vFFR without the need to model blood flow throughout the entire coronary tree. One solution to this problem is the use of a fixed volume flow rate in all patients [14]. However, there is a significant variation in inlet volume flow rate that is mostly dependent on the location of stenosis. Other attempts to determine individualized flow rates proved to be quite time consuming and requires administration of adenosine to acquire hyperemic angiographic images. Another group of investigators measured contrast medium transport time in the reconstructed vessel on hyperemic projections. The mean volume flow rate at hyperemia was then derived using the lumen volume of the reconstructed coronary tree divided by the mean transport time [49]. This approach enables individualized quantification of inlet volume flow rate for patients compared to a "one-size-fits-all" strategy.

\subsection{Residence Time}

A plug flow reactor (PFR) is considered an unmixed system, whereas a continuous stirred-tank reactor (CSTR) delivers ideal mixing [56]. But in reality, reactors have stagnant regions (dead zones) which are regions with suboptimal mixing of materials.

Residence Time Distribution (RTD) analysis is a diagnosis metric that can be used for inspecting chemical reactors. It is useful in modeling reactors as well as estimation of effluent properties. This technique is particularly advantageous when mixing of materials in such reactors is non-ideal [56]. MacMullin and Weber [57] for the first time proposed the idea of using RTD for analyzing chemical reactor efficiency. However, it was Dankwerts who suggested the concept of mean residence time [58]. For example, deficient agitation in CSTRs 
could be studied using RTD. For experiment design, transparent reactors are used with both colored tracers and a colored reaction, so that investigators can easily observe the evolution of tracer and reactant concentrations in the reactor during reaction time; additionally, the reaction can be recorded if desired. RTD calculation could be applied for the experimental characterization of the flow pattern in different systems such as packed bed and tubular reactor. The RTD function, $\mathrm{E}(\mathrm{t})$, measures the time for different functions of fluid element residing in the reactor (macromixing). $\mathrm{E}(\mathrm{t})$ function can be calculated experimentally for a real reactor using methods of tracer injection. If a tracer impulse is injected at the inlet, and tracer concentration $\mathrm{C}_{\mathrm{T}}(\mathrm{t})$ at outlet is measured as a function of time, the experimental RTD function could be expressed as follows [56]:

$$
E(t)=\frac{C_{T}(t)}{\int_{0}^{\infty} C_{T}(t) d t}
$$

Also, the effective mean residence time is $\bar{t}$ :

$$
\bar{t}=\int_{0}^{\infty} t E(t) d t
$$

Effective mean residence time is compared to nominal mean residence time which is calculated by the ratio of volume to volume flow rate. RTD can detect by-pass and/or dead volume formation within the real reactor. In order to diagnose a defective function in real reactors, a comparison between RTD for ideal reactor and non-ideal reactor is needed. Residence time distributions functions for the ideal flow patterns of plug flow and perfect mixing in CSTR are [56]:

Ideal plug flow (Figure 2.2 a):

$$
E(t)=\left\{\begin{array}{lll}
0 & \text { if } t \neq \bar{t}_{r} \\
\infty & \text { if } t=\bar{t}_{r}
\end{array}\right.
$$


Perfect mixing reactor (Figure $2.2 \mathrm{~b}$ ):

$$
E(t)=\frac{e^{-t / \bar{t}_{r}}}{\bar{t}_{r}}
$$

Where $\bar{t}_{r}$ is the average residence time.

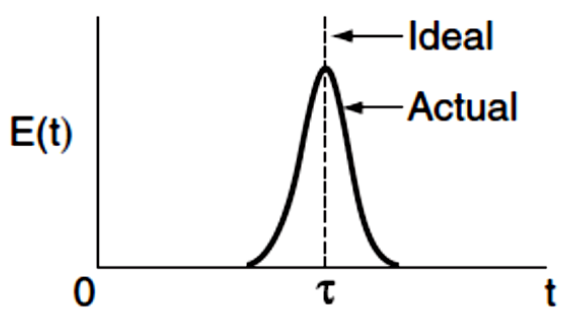

(a)

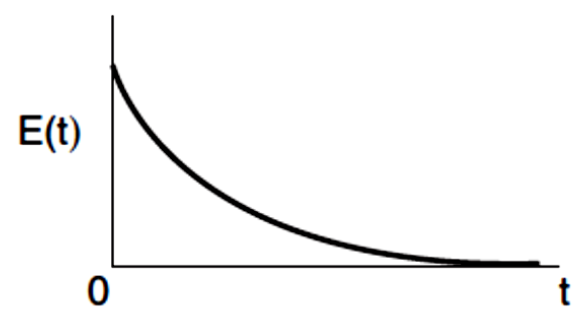

(b)

Figure 2.2 (a) RTD for near plug flow reactor and (b) RTD for near perfectly mixed reactor [89].

In summary, the RTD can be used to examine and diagnose the type of mixing, bypassing and dead zones in a non-ideal reactor that is currently on stream however is unable to predict the efficiency of conversion using ideal reactor models.

\subsection{Mean Age Theory}

Mean age theory provides a computationally efficient method for computing residence time or "age" of fluid, where "age" refers to the amount of time a parcel of fluid resides between two boundaries. In 1953, Danckwerts [58] mathematically presented the residence time distribution function and used the function for measuring the amount of time spent in the vessel. He tested this concept for different systems including packed bed flow, pipeline flow and flow through a stirred tank. 
Later on, Danckwerts [59] proposed the concept of residence time distributions for different locations in a vessel. He called this new value 'age'; the age at the outlet equals residence time. Then, Sandberg [60] derived the steady scalar transport equation for mean age based on the work of Spalding [61] which, provided much of the groundwork required for deriving a transport equation for mean age (the first moment of age). Nonetheless, limitations in computing power at the time made practical use of mean age impossible for decades. Spalding defined mean age as:

$$
a(x)=\frac{\int_{0}^{\infty} t C(x, t) d t}{\int_{0}^{\infty} C(x, t) d t}
$$

Where $\mathrm{C}$ is the concentration of a hypothetical scalar at location (x) and time (t). Sandberg [60] brought it to its conclusion a transport equation for mean age which is based on the advective-diffusive equation:

$$
\frac{\partial C}{\partial t}+\nabla \cdot(u C)=\nabla \cdot(D \nabla C)
$$

Developing this equation with mean age definition and the recognition that age and time are related leads to the conclusion:

$$
u \nabla a=\nabla \cdot D \nabla a+1
$$

This final equation provides the transport for mean age, rather than concentration of a scalar. Essentially, a scalar constant source term replaced the unsteady term. This is discriminating, in that one unit of mean age should equal for every unit of time passed.

The boundary conditions were shown to be for the same concentration:

$$
\mathrm{a}=0 \quad \text { Inlet }
$$




$$
\begin{array}{ll}
\frac{\partial a}{\partial X_{n}}=0 & \text { Outlet } \\
\frac{\partial a}{\partial X_{n}}=0 & \text { Wall }
\end{array}
$$

Baleo and le Cloirec [62] attempted to experimentally validate the mean age transport equation in CFD. They used a turbulent flow model in a pipeline. They validated the mean age equation by determining mean age spatial distribution using numerical computation. In 2010 , Liu and Tilton [63] presented the higher moments of age concept for the first time. Their study demonstrated the advantage of mean age calculation using the spatial distributions which can be exported from CFD simulations. Liu [64] used mean age theory for determining mixing degree in an open system with continuous flow. Although Danckwerts [59] had proposed mixing degree calculation and Zwietering [65] had further developed this concept without using CFD, Liu quantified mixing using CFD for the first time. Russ and Berson [66] extended mean age theory to multiple phases and validated it with a water-oil flow system using CFD. 2.7 Residence Time Applications in Cardiovascular Research

Conventional residence time theory is a measure of flow distribution at the exit of a continuous system whereas mean age theory provides spatial resolution throughout the interior of the flow domain. Mean age has been demonstrated in a variety of applications for both single phase $[62,63]$ and multiphase flow $[66,67]$. Multiphase would be beneficial if, for example, one sought to determine the age of blood components such as cells, platelets, and plasma, etc. independent of each other. Residence time determination has extensively been used in cardiovascular applications. Kunov et al. [68] showed experimentally, using tracer particles, that volumetric residence times were elevated in the separation zone distal to arterial stenosis. Cao et al. [51] measured increasing residence time of tracers downstream of stenosis using a 
laser light sheet flow visualization method with pseudo-color display. CFD has been extensively used in cardiovascular hemodynamics research to determine residence time, wall shear stress, etc. Below, a brief overview of cardiovascular applications of residence time computation based on principles of CFD is presented.

König et al. [69] developed a tool for experimental validation of numerical simulations of blood flow mixing and fluid residence time in a ventricular assist device (VAD). The tool allowed determination of flow penetration, recirculation regions and extent of mixing and residence times in successive cycles of fluid entering in a given cycle. They demonstrated that the simulations are capable of qualitative characterization of flow mixing; fluid residence times quantification was less accurate.

Ford et al. [70] simulated aneurysmal blood flow dynamics by coupling threedimensional (3-D) cine X-ray angiography and CFD in order to calculate residence time. There are no techniques for adequately measuring complex blood velocity fields in vivo. They describe how cine X-ray angiograms may be simulated for the purpose of indirectly validating patient-specific CFD models. Virtual angiographic images and residence time contours were derived from an image-based CFD model of aneurysm artery. There was an excellent correlation between clinical images and residence time contours.

Bernsdorf et al. [71] developed a Lattice Boltzmann method to simulate blood clot formation using residence time modeling. They used an adaptation of the Lattice Boltzmann method for simulating blood coagulation within a stenosed artery. They estimated the residence time of fluid by a passive age scalar; the inlet flow age was defined zero. The majority of fluid passed through the artery without increased age for clotting. However, a part of the fluid trapped within recirculation zones produced increased age of the whole fluid. This stagnant 
blood that remains in such regions of recirculation have the potential for thrombus formation. Therefore, determination of fluid residence time could be an important factor for predicting clotting.

Gay and Zhang [72] performed numerical analysis of pulsatile blood flow for unobstructed, stenosed, and stented carotid arteries in order to identify hemodynamic factors in the initiation and growth of stenosis leading to severe occlusion of a diseased artery. They performed modeling and analysis of two-dimensional unsteady pulsatile flows that are most relevant in the context of thrombus formation. Their results provide detailed quantitative analysis on blood flow pattern, wall shear stress, particle residence time, and oscillatory shear index. The analysis of particle residence time culminates in a better understanding of blood clot formation and why blood clot forms in a stenosed and a stented carotid artery.

Rayz et al. [73] investigated the effect of increased flow residence time by modeling passive scalar advection in the same aneurysmal geometries. They used a new postprocessing technique, referred to as "virtual ink" based on the passive scalar distribution maps for visualizing the flow and estimating the flow residence time. The virtual ink technique clearly depicted regions of flow separation. They calculated flow residence time at different locations adjacent to aneurysmal walls and correlated them with areas of clot formation observed on MRI. They demonstrated a significant relationship between regions where CFD predicted either an increased residence time or low wall shear stress and the regions where thrombus deposition was observed to occur in vivo.

Suh et al. [74] presented qualitative and quantitative results of particle residence time computation, using magnetic resonance imaging (MRI) and CFD, to explain the complex hemodynamics in eight different abdominal aortic aneurysms. They used phase-contrast MRI 
to measure blood flow to estimate physiologic boundary conditions. Next, they quantified flow stagnation and recirculation in aneurysms by computing particle residence time. This study reported significant regional differences in particle residence time in abdominal aortic aneurysms including a long-duration particle residence time region localized in the aneurysm that could represent flow stagnation and recirculation zone with elevated probability of thrombus formation.

Long et al. [75] proposed a continuum-based model of particle residence time for moving structure fluid mechanics and fluid structure interaction (FSI) computations. They showed that residence time is a quantity of clinical interest for blood pumps because it correlates with thrombotic risk. Particle residence time quantification was used in the VAD design with the goal of reducing thrombotic risk.

Arzani [76] used blood cell residence time for better understanding of blood rheology in aneurysmal flows. Experimental data suggest that red blood cell aggregation and rouleaux formation require notable red blood cell residence-time in a low shear rate regime. He proposed a novel hybrid Newtonian and non-Newtonian rheology model where the shear-thinning behavior is activated in high residence time regions based on experimental data. He used Lagrangian particle tracking to define a backward particle residence-time measure and detect stagnant regions with increased rouleaux formation possibility. This work demonstrated that non-Newtonian models could be used in simulation of blood flow in large arteries with similar results to Newtonian models provided that there are no regions of high backward residence time and low shear rate.

Reza and Arzani [77] showed that flow stagnation and residence time were important variables of diseased arterial flows that are effective on thrombosis. Residence time was 
calculated using Eulerian and Lagrangian models. Each of these methods has a different definition of residence time, and it is not clear how they are related. They considered imagebased computational models of blood flow in an abdominal aortic aneurysm and a cerebral aneurysm and residence time was calculated using different methods. In the Lagrangian methods, they used discrete particle tracking of massless tracers to calculate particle residence time and mean exposure time. In the Eulerian methods, transient transport models were used to quantify residence time using Eulerian residence time and virtual ink approaches. Their results highlighted that most residence time methods have a conceptually distinct definition of residence time and therefore should be utilized depending on the specific application of interest.

CFD is also used to quantify other hemodynamic parameters used in cardiovascular sciences such as relative residence time, wall shear stress, oscillatory shear index, wall shear stress exposure time, etc. Himburg et al. [78] introduced relative residence time (RRT) to investigate the effect of the residence time of solutes and formed elements of the blood on atherosclerotic process. Notably, RRT is indirectly computed from time-averaged wall shear stress (WSS) and oscillatory shear index (OSI) rather than directly from tracer measurements. Dong et al. [79] presented a method to detect diseased carotid bifurcation using flow analysis and virtual intervention. They calculated time-averaged WSS, OSI, and RRT, and assessed atherosclerosis burden from a hemodynamic standpoint. They demonstrated that oscillatory shear index, and relative residence time can be used for computational modelling of downstream vascular impedance when the outlets boundary conditions are not available.

Arzani et al. [80] compared wall shear stress exposure time (WSSET) against the more common RRT measure and WSS divergence measure, in six patient-specific abdominal aortic 
aneurysm, two carotid arteries, a cerebral aneurysm, and a coronary aneurysm. Variables such as WSSET that quantify near-wall transport are important in several cardiovascular pathologies whose progression are influenced by hemodynamics. Their work suggests that WSSET, compared to RRT, better approximates the locations of near-wall stagnation and concentration buildup of chemical species, particularly in complex flows that are ubiquitous in blood hemodynamics.

Malota at al. [81] hypothesized that selected hemodynamic indices such as the OSI, residence time index, pressure drop coefficient that are induced by change of blood flow rate, heart rate and vessel geometry may be beneficial in evaluating hemodynamic significance of coronary stenosis and the risk of stenosis progression. The variable flow was calculated for vessels both with stenosis of 10 to $95 \%$ and without stenosis. They demonstrated that the degree of stenosis has a significant impact on OSI and RRT; the maximum OSI and RRT appeared in an area downstream of stenosis where there also were the minimum values of WSS. Therefore, RRT and OSI may be useful to assess hemodynamic significance of coronary stenosis. 


\section{CHAPTER THREE \\ EXPERIMENTAL METHODS}

\subsection{Purpose}

The purpose of this research was to develop a novel non-invasive method to assess coronary artery stenosis. The method utilizes coronary angiography imaging without the invasive FFR measurement in conjunction with an accurate, highly sensitive computational model that allows the cardiologist to make an informed decision regarding stent placement. Coronary blood flow across stenotic coronary lesions is modeled with CFD to assess stenosis severity according to the newly developed and proprietary metric, Blood $\mathrm{RT}_{\mathrm{R}}$, which was then correlated with the gold standard FFR metric. A proprietary key model input, blood flow rate specific to the site of each lesion, was developed using multiple linear regression. Notably, this method delivers the benefits of FFR measurement in terms of improvement in patient outcomes and cost savings, while simultaneously avoiding the disadvantages such as discomfort to the patients and cost and time burden for the doctors and hospitals.

\subsection{Plan}

Firstly, a new non-invasively determined metric, dimensionless Blood ${ }_{\mathrm{RT}}$, to assess the physiological significance of coronary artery stenosis analogous to the coronary stenosis assessment based on FFR is presented. The method to determine the metric is based on CFD in conjunction with patient specific imaging to compute mean residence time of blood passing through stenotic coronary artery segments. A threshold for Blood $\mathrm{RT}_{\mathrm{T}}$ was determined based on 
the sensitivity and specificity of this metric to establish diagnostic accuracy compared with the gold standard pressure wire FFR threshold. The metric was then tested in one hundred coronary arteries with known pressure-wire FFR for clinical validation.

Secondly, the method was applied to determine the contribution of each stenosis to the overall Blood $_{\mathrm{RT}}$ when multiple stenoses was present in series. Blood $\mathrm{RT}_{\mathrm{T}}$ was calculated for each stenosis and then for the overall series in three patients with serial stenoses. The Blood $\mathrm{RT}$ is uniquely better suited to do this compared to invasive FFR. Blood ${ }_{\mathrm{RT}}$ depends on time, which can be defined without influence of upstream residence time, whereas pressure depends on the history of flow, making it difficult to calculate FFR for each individual stenosis.

Thirdly, an individualized, patient-specific value for blood volume flow rate at the segment inlet would presumably increase the accuracy of assessment for Blood $\mathrm{RT}_{\mathrm{T}}$ as well as virtual FFR compared to current estimated or empirical boundary conditions. Therefore, the inlet blood volume flow rate was determined in 100 coronary arteries with stenosis using multiple linear regression. The reference standard, against which the results were tested, is a patient specific inlet volume flow rate computed from invasively measured FFR values. The method was then validated using a separate cohort of 100 patients.

\subsection{First Aim. Blood Residence Time to Assess Significance of Coronary Artery Stenosis}

\subsubsection{Patient Population}

One hundred arteries from ninety patients who had undergone coronary angiography and FFR measurements for clinical indications in two hospitals affiliated with the University of Louisville were retrospectively included in this study. Patients' characteristics are detailed in Table 3.1. Patients with stenosis in a major epicardial artery (left anterior descending artery [LAD], left circumflex [LCx]/obtuse marginal $[\mathrm{OM}]$ and right coronary artery [RCA]) were 
eligible for inclusion in the study. Exclusion criteria were: significant ostial left main or ostial RCA disease, coronary arteries with bifurcational lesions, and coronary arteries distally protected by bypass grafts. All lesions included in the study had documented adenosine administration and pressure-wire FFR recording, as well as suitable angiographic projections for three-dimensional (3D) reconstruction. The institutional review board approved the study protocol used for patient cases.

Table 3.1 Clinical characteristics of patients

\begin{tabular}{|l|l|}
\hline Total Patients & 90 \\
\hline Total vessels & 100 \\
\hline Age & $63.3 \pm 27.6$ \\
\hline Male gender & $57(63.3 \%)$ \\
\hline Hypertension & $87(96.6 \%)$ \\
\hline Diabetes mellitus & $40(44.4 \%)$ \\
\hline Current smoker (last 1 year) & $34(37.7 \%)$ \\
\hline History of prior myocardial infarction & $35(38.9 \%)$ \\
\hline History of prior PCI & $42(46.6 \%)$ \\
\hline History of prior CAD & $57(63.3 \%)$ \\
\hline Hyperlipidemia & $74(82.2 \%)$ \\
\hline family history & $29(32.2 \%)$ \\
\hline Vessel disease & $83(83 \%)$ \\
\hline Single-vessel & $4(4 \%)$ \\
\hline Two-vessel & \multicolumn{2}{|l}{} \\
\hline
\end{tabular}




\begin{tabular}{|l|l|}
\hline Three-vessel & $3(3 \%)$ \\
\hline total vessels & 100 \\
\hline
\end{tabular}

\subsubsection{D Rendering}

Three-dimensional reconstruction of coronary arteries was performed with the CAAS 7.5 QCA-3D system (Pie Medical Imaging, Maastricht, The Netherlands) [14]. In brief, two two-dimensional (2D) angiographic images encompassing the stenosis of interest, in images $30^{\circ}$ apart, were used to generate a 3D rendering of the segment of interest, in the end-diastolic frames (Figure 3.1).
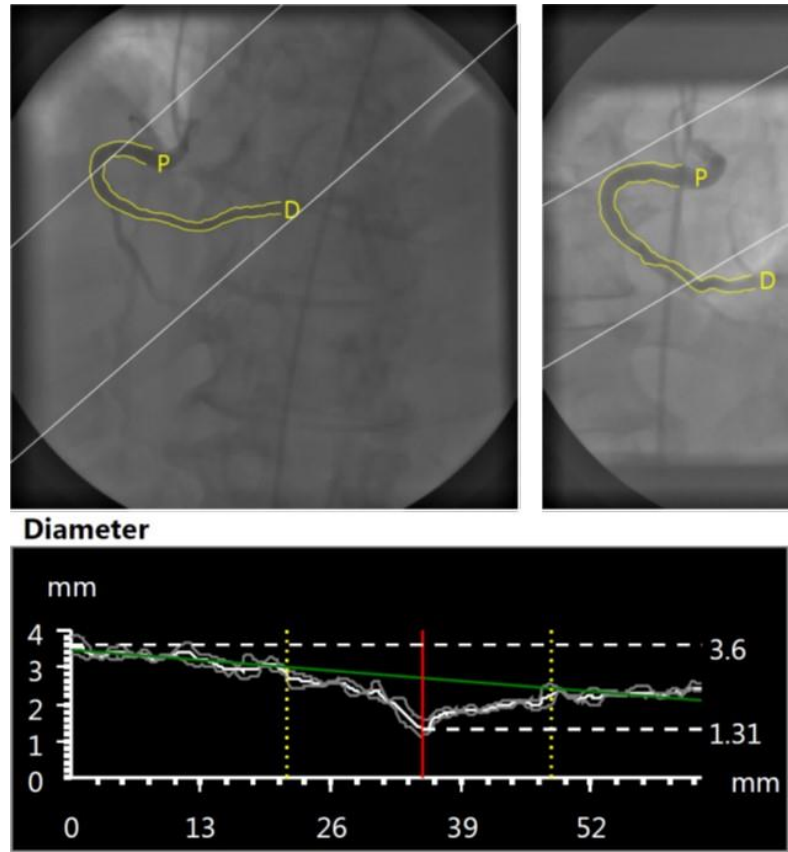
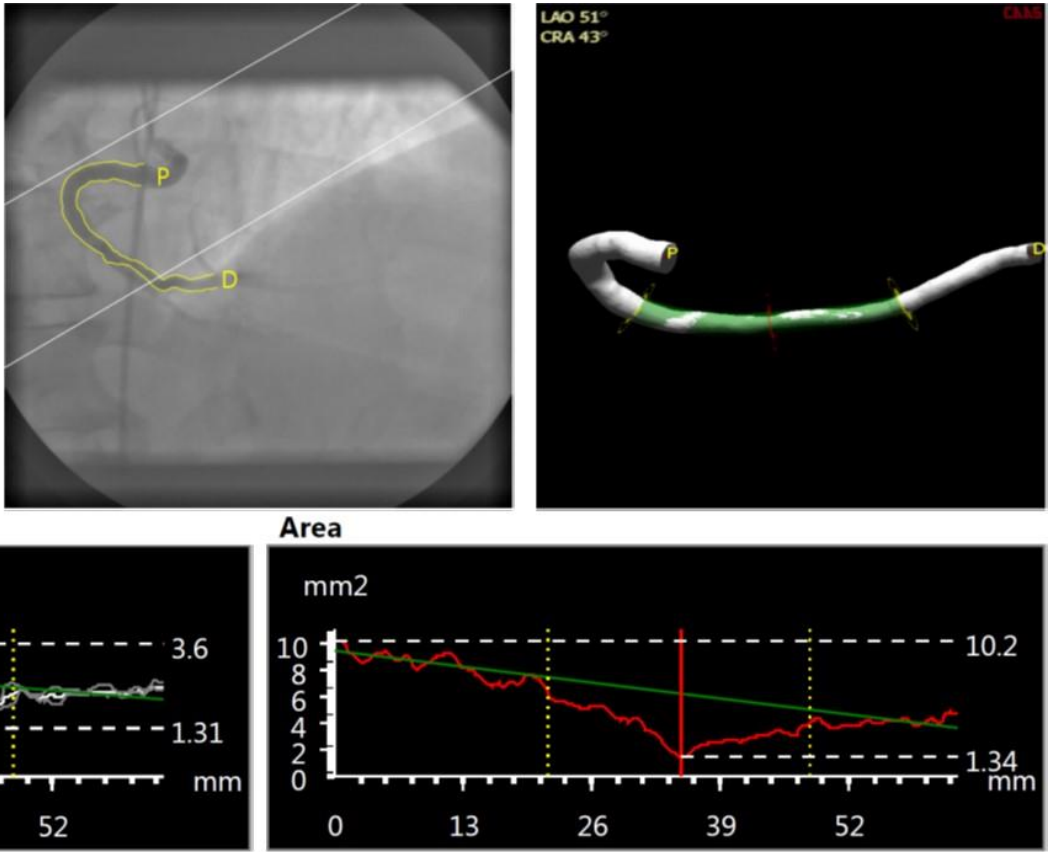

Figure 3.1 3D rendering steps and diameter and area results.

\subsubsection{CFD Modeling}

Blood flow in coronary arteries was simulated using ANSYS Fluent 17.0. Reynolds numbers were between 128-1501 in the region of stenosis, so flow was modeled as Laminar 
[29]. Blood density was $1045 \mathrm{~kg} \cdot \mathrm{m}^{-3}$ [82]. Previous studies show shear rate in coronary arteries is in the $400 \mathrm{~s}-1$ range [83] and the corresponding viscosity is Newtonian for that shear rate [84], so blood viscosity was modeled according to Newtonian viscosity using each patient's measured viscosity. Unstructured computational meshes were built as tetrahedral shaped cells using ANSYS Mesher 17.0 (Figure 3.2). An optimal node count of 542,000 was determined by mesh sensitivity analysis of mean residence time for an artery with volume of $4.04 \times 10^{-8}$ $\mathrm{m}^{3}$, and then scaled accordingly for the size of each case. Once the flow field was established, mean residence time and then dimensionless Blood $_{R T}$ was computed using mean residence time-based computations for each time increment and averaged over the duration of one complete pulse.
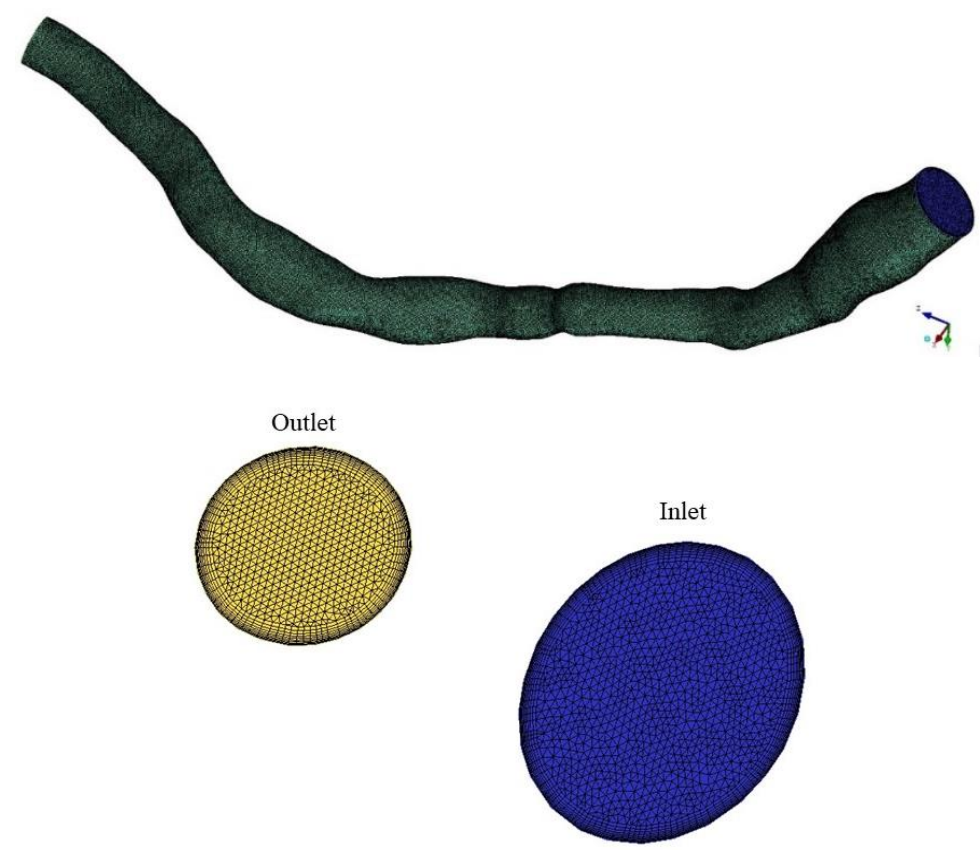

Figure 3.2. Mesh generation for a coronary artery, inlet, and outlet.

The inlet boundary condition was a transient velocity waveform (Figure 3.3a) representing the coronary blood cycle as previously published [85]. The outlet boundary 
condition was a pressure waveform (Figure 3.3b). Both were scaled to match the mean flow in hyperemic conditions and pressure measured for each patient, and then programmed using user defined functions. Similar to node count, a sensitivity analysis determined an optimal time step size of $0.01 \mathrm{~s}$.
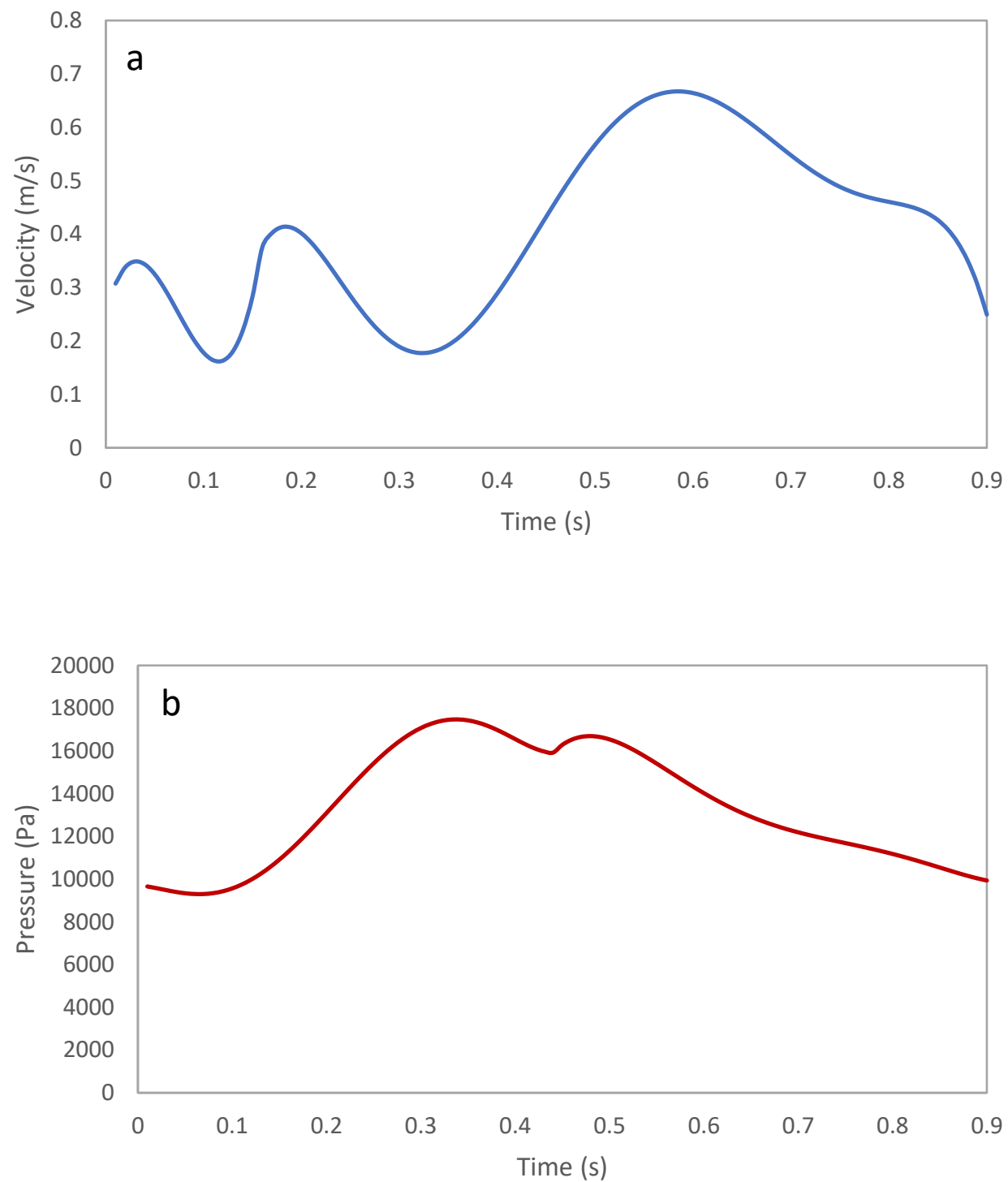

Figure 3.3 Hyperemic boundary conditions: (a) inlet velocity (b) outlet pressure. 
Below is a step by step procedure for setting up the model and mean age calculation in Fluent:

Modeling Flow Procedure:

1. Import Mesh in Fluent

2. General:

- $\quad$ select transient

3. Models:

- Single Phase

- Viscous-Laminar

4. Materials:

- Add blood properties; Density:1045 kg/m3 and Viscosity: 0.0035 kg/(m.s)

5. Boundary Conditions:

- Inlet=>Velocity Inlet (User-Defined Function (UDF)), Outlet=>Pressure Outlet (UDF)

6. Solution Methods:

- Pressure-Velocity Coupling => Phase Coupled SIMPLE

- $\quad$ Gradient => Least Squares Cell Based

- Spatial Discretization => first order

- Momentum=> first order upwind

- Volume Fraction=> First order upwind

- User Scalar 0 => First order upwind

- User Scalar 1 => First order upwind

7. Solution Controls:

- Explicit Relaxation Factors: 
- Pressure: 0.1

- Density:0.3

- Body Forces:0.3

- Momentum: 0.3

- Volume Fraction:0.5

- Granular Temperature: 0.2

- User Scalar 0: 0.1

8. Run Calculation:

- $\quad$ Time Step Sizes (s): 0.01

- Number of Time Steps: 720

- Max Iterations/Time Step: 50

To measure mean time according to mean age theory:

Mean age is computed as a User Defined Scalar [66].

1. Select Define $\rightarrow$ User-defined $\rightarrow$ Scalars

2. Change the Number of User Defined Scalars to ' 1 '

3. Uncheck 'Inlet Diffusion'

4. Flux function is 'mass flow rate' (for any boundary condition).

5. Ensure that 'default' is selected next to Unsteady Function

6. Click 'OK'

7. Select your material under 'Materials' on the left

8. Next to UDS Diffusivity, ensure that 'defined-per-uds' is selected in the drop-down and click 'edit'

9. Enter a very low value ( 6e-9) and click 'OK' 
10. Click 'Change/Create'

11. Click 'Close'

12. Select 'Cell Zone Conditions' on the left

13. Select the zone in which you do NOT want to calculate mean age

14. Check 'Fixed Values'

15. Next to 'User Scalar 0' select 'constant' in the drop down

16. Enter ' 0 ' for the value

17. Click 'OK'

18. Select the zone in which you DO want to calculate mean age

19. Check 'Source Term'

20. Click 'edit' next to 'user scalar 0'

21. Choose 'constant' from the drop-down

22. Enter 'density' for the value

23. Click ' $\mathrm{OK}$ '

24. Select 'Boundary Conditions' on the left

25. Select your inlet boundary

26. Choose the 'UDS' tab

27. Select 'fixed value' from the boundary condition pane

28. Input ' 0 ' for the boundary value

29. Click 'OK'

30. Run your simulation

UDF functions were employed for defining the inlet velocity and outlet pressure pulses:

\#include "udf.h" 


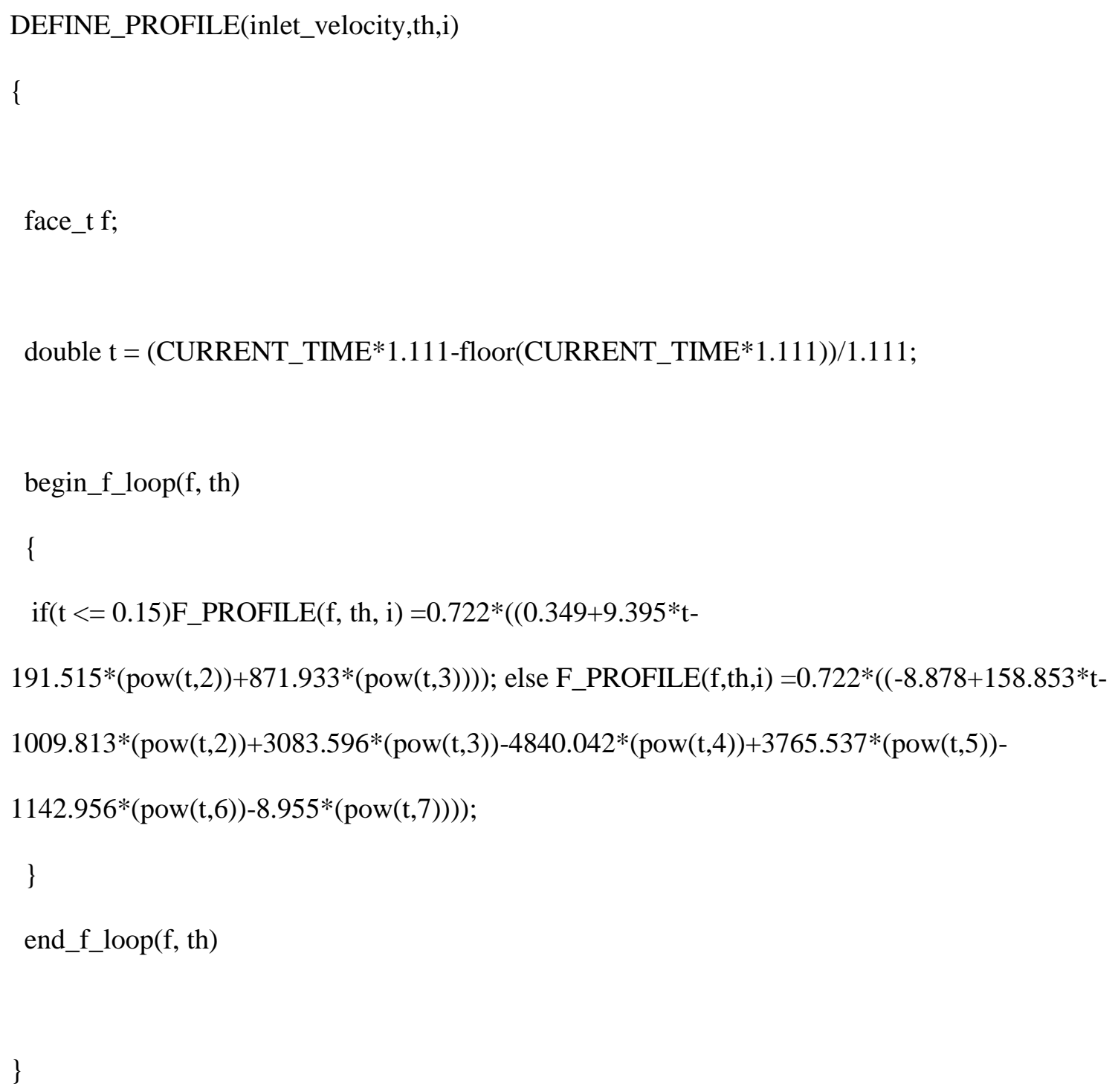




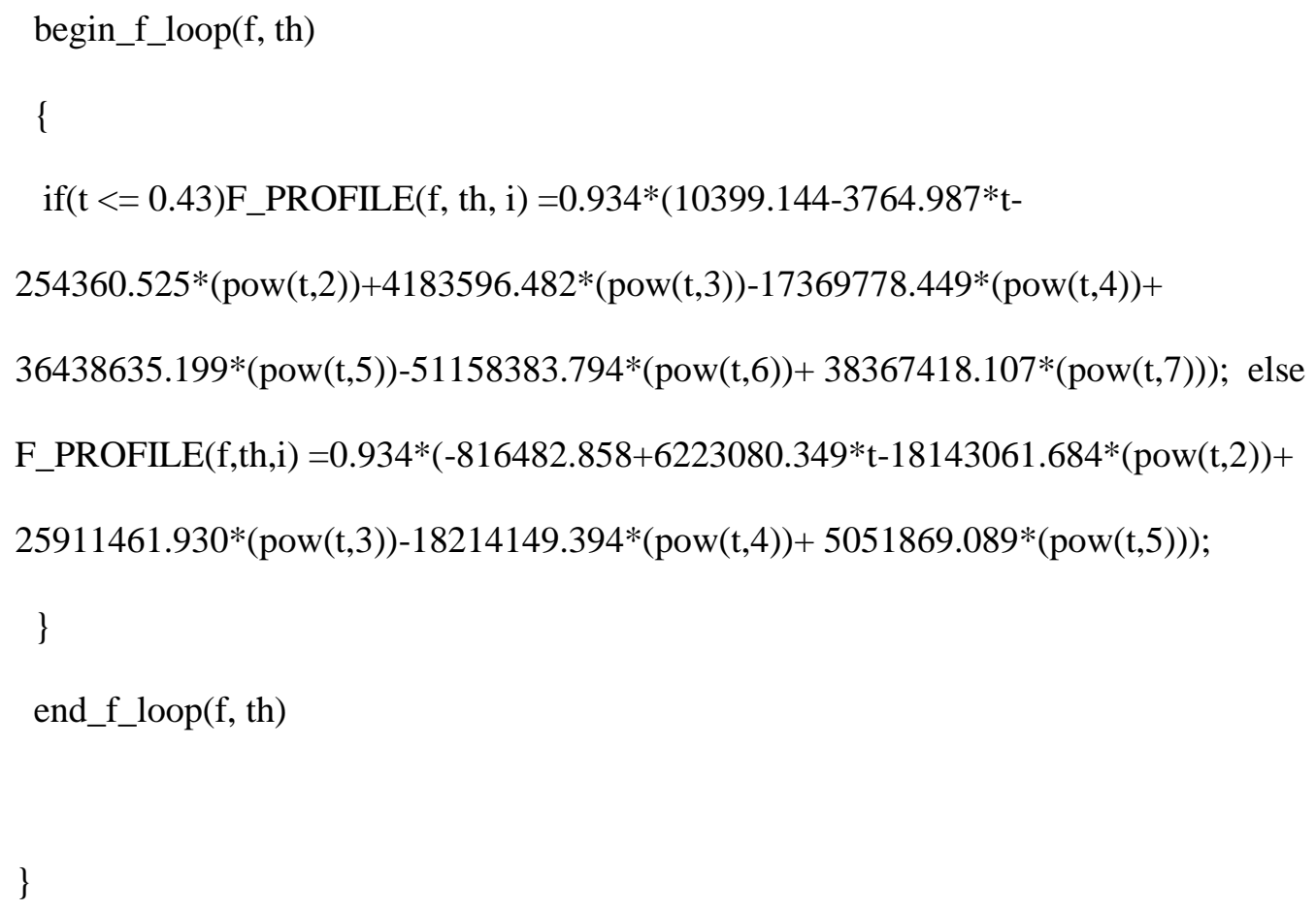

The UDF was altered for each patient based on each patient's flow rate by scaling each coefficient according to each patient's inlet velocity or outlet pressure. The UDF is implemented in Fluent:

User-Defined: Functions $=>$ Interpret

Mean residence time must be calculated with good stability. For a stabilized result, under the relaxation factor of scalar 0 was reduced from 1 to 0.1 . The computation was run until two or three stable pulses were obtained. Figure 3.4 shows an example where it took nine 0.9 second pulses to achieve a stable pulse of residence times. The vertical orange line shows the beginning of stability. There would not be a significant change from that point forward. The mean residence time was defined as the average over one complete pulse. 


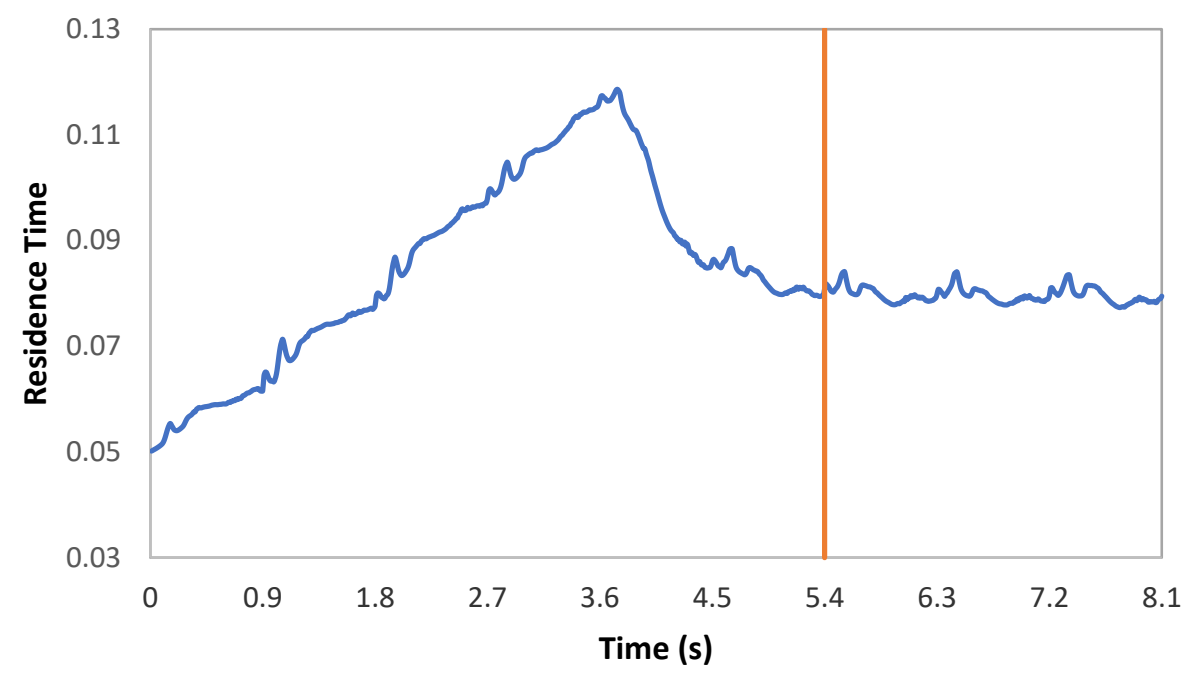

Figure 3.4 Stability of residence time pulses.

\subsubsection{Statistical Analysis to Determine Blood ${ }_{\mathrm{RT}}$ Threshold}

Observations were grouped into two groups, abnormal pressure-wire FFR $(<=0.80)$ and normal pressure-wire FFR (>0.80). SPSS 20.0 software was used for statistical analysis. Receiver operator characteristic (ROC) curve analysis was performed. Sensitivity and specificity of Blood $\mathrm{RT}_{\mathrm{T}}$ were calculated along with their $95 \%$ confidence intervals using logistic regression analysis to determine the optimal threshold for Blood ${ }_{\mathrm{RT}}$. Except for the patient characteristics, analyses were performed on a per-vessel basis. A two-sided p-value $<0.05$ was considered significant. The correlation between Blood $_{R T}$ and FFR was studied using the Pearson (r) correlation coefficient.

3.4 Second Aim. Assessment of Significant Stenosis for Coronary Artery with Serial Stenoses 3.4.1 3D Rendering and Patients' Arteries

In this study, three left anterior descending (LAD) coronary arteries were selected. Patient A has two serial stenoses and patient B and C each have three serial stenoses that blue lines separate each stenosis for calculation individual Blood ${ }_{R T}$ and yellow arrows show place 
of each stenosis (Figure 3.5). The volume of the coronary arteries are 1.77E-07, 3.18E-07 and 1.55E-07 $\mathrm{m}^{3}$, respectively. Patient characteristics are detailed in Table 3.2.
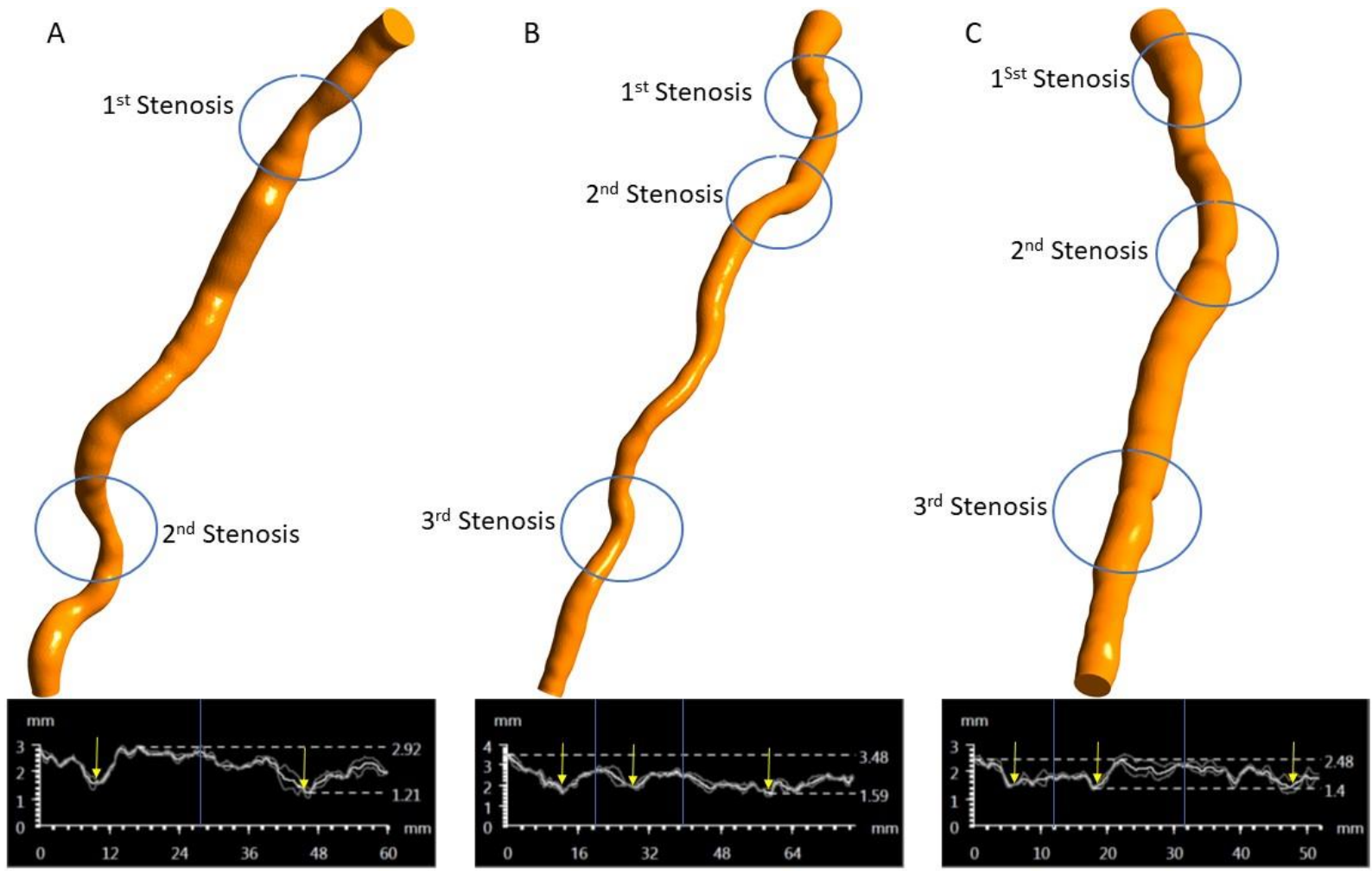

Figure 3.5 3D-rendering patient's coronary arteries from angiography.

Table 3.2 Characteristics of patients enrolled in the study.

\begin{tabular}{|l|l|l|l|}
\hline & Patient A & Patient B & Patient C \\
\hline Age & 59 & 66 & 45 \\
\hline Gender & Male & Male & Male \\
\hline History of prior myocardial & No & No & Yes \\
\hline infarction & & & \\
\hline History of prior CAD & No & No & Yes \\
\hline History of prior PCI & No & No & Yes \\
\hline
\end{tabular}




\begin{tabular}{|l|l|l|l|}
\hline Diabetes mellitus & Yes & No & No \\
\hline Hypertension & Yes & Yes & Yes \\
\hline Current smoker (last 1 year) & Active & Former & Former \\
\hline Segment & Mid LAD & LAD & Mid LAD \\
\hline Volume flow rate & $1.72 \mathrm{E}-06$ & $2.48 \mathrm{E}-06$ & $1.62 \mathrm{E}-06$ \\
\hline Volume & $2.45 \mathrm{E}-07$ & $3.18 \mathrm{E}-07$ & $1.54 \mathrm{E}-07$ \\
\hline
\end{tabular}

\subsubsection{CFD Modeling}

CFD model inputs were the same as in 3.3.3. The optimal node count was $1,518,868$, $1,499,578$ and 808,170 nodes based on using the sensitivity analysis of mean residence time. The nominal mean residence time equals volume artery divided by volume flow rate. The Blood $_{\mathrm{RT}}$ was computed through mean age theory in coronary systems as described in 3.3.3. FFR was calculated for each stenosis by dividing distal pressure to proximal pressure of the stenosis. Blood $\mathrm{RT}_{\mathrm{R}}$ is defined as a dimensionless number to account for varying length and volume of each arterial segment plus varying blood flow rates as follow:

$$
\text { Blood }_{R T}=\left(\frac{\text { Nominal Mean Residence Time }(s)}{\text { Mean Residenc Time }(s)}\right)
$$

Thus, Blood $\mathrm{RT}_{\mathrm{T}}$ indicates the prolongation of Blood $\mathrm{RT}_{\mathrm{T}}$ in stenotic coronary arteries compared to a normal artery. 


\subsection{Third Aim. Volume Flow Rate Determination Using Machine Learning}

\subsubsection{Regression Model to Determine Blood Flow Rate}

The actual blood volume flow entering a stenotic segment was determined by backcalculating from invasively determined FFR for each patient. The back-calculation was performed with trial and error of flow rates as the inlet boundary condition in CFD until the virtual FFR equaled the invasive FFR. With the known actual flow rate, multiple linear regression using SPSS 20.0 software determined blood flow rate from six factors. One hundred coronary arteries from patients with coronary stenosis were used to develop the regression. The factors included in the regression model include coronary segment type (A), inlet diameter of coronary segment (B), stenosis diameter (C), stenosis percentage (D), inlet area of coronary segment $(\mathrm{E})$, and stenosis area $(\mathrm{F})$. The reference standard, against which the results were tested, is the patient specific back-calculated inlet volume flow rate.

Building a robust regression model that takes into account the intrinsic property of the training data as well as the impacts of the different perturbations it can undergo requires aggregating multiple regression models. This approach, known as ensemble learning, has been investigated in many studies and promising results have been reported $[86,87]$. The advantage of ensembles compared to single models has been reported in terms of increased accuracy and robustness [88]. Even though most of the previous work has focused on classification tasks, they still can be applicable to regression problems by using appropriate adjustments [89]. An ensemble learning approach, usually known as the overproduce-and-choose [90], includes three main steps. First, an ensemble of single models is generated using homogeneous or heterogeneous algorithms (e.g. using some of the regression algorithms mentioned above). Second, some of the learned models are pruned in order to improve the prediction accuracy 
and to reduce the computational costs. This step is similar to feature selection where feature values are replaced with values predicted by the different models. Thus, any of the techniques outlined above could be used for this task. The last step of ensemble learning consists of integrating the selected base models to obtain the prediction of the ensemble for new cases. For regression ensembles, the integration step is often performed using a weighted linear combination of the predictions. The weighting function can be a constant or a non-constant, depending on the integration approach. The model for determining inlet blood volume flow rate was assessed in a separate validation cohort of 100 patients. Statistical analysis was same 3.3.4 section. 


\section{CHAPTER FOUR}

\section{BLOOD RESIDENCE TIME TO ASSESS SIGNIFICANCE OF CORONARY ARTERY}

\section{STENOSIS}

\subsection{Pathlines}

Blood flow pathlines are shown in two left anterior descending (LAD) artery segments as representative examples of one case above and one below the FFR threshold (Figure 4.1a, 1b). Patient A had a non-significant stenosis with FFR equal to 0.94 and patient B had a significant stenosis with FFR equal to 0.63. Pathlines remain relatively ordered for Patient A during both systole (at $0.15 \mathrm{~s}$ of the pulse) and diastole (at $0.70 \mathrm{~s}$ of the pulse), while pathlines reveal a small but noticeable region of low velocity recirculation and holdup distal to the stenosis, especially during diastole (Figure 4.1b). In Patient A, the maximum velocity during diastole was only about $\sim 40 \%$ greater than the inlet velocity $(\sim 1.0 \mathrm{~m} / \mathrm{s}$ compared to $\sim 0.72 \mathrm{~m} / \mathrm{s})$ at this point in the pulse input (Figure 1a shows the velocity input pulse for Patient A), while

for Patient B the maximum velocity was about $650 \%$ greater than the inlet velocity at this point ( $\sim 3 \mathrm{~m} / \mathrm{s}$ compared to $\sim 0.4 \mathrm{~m} / \mathrm{s}$ ) in the pulse (pulse not shown). 

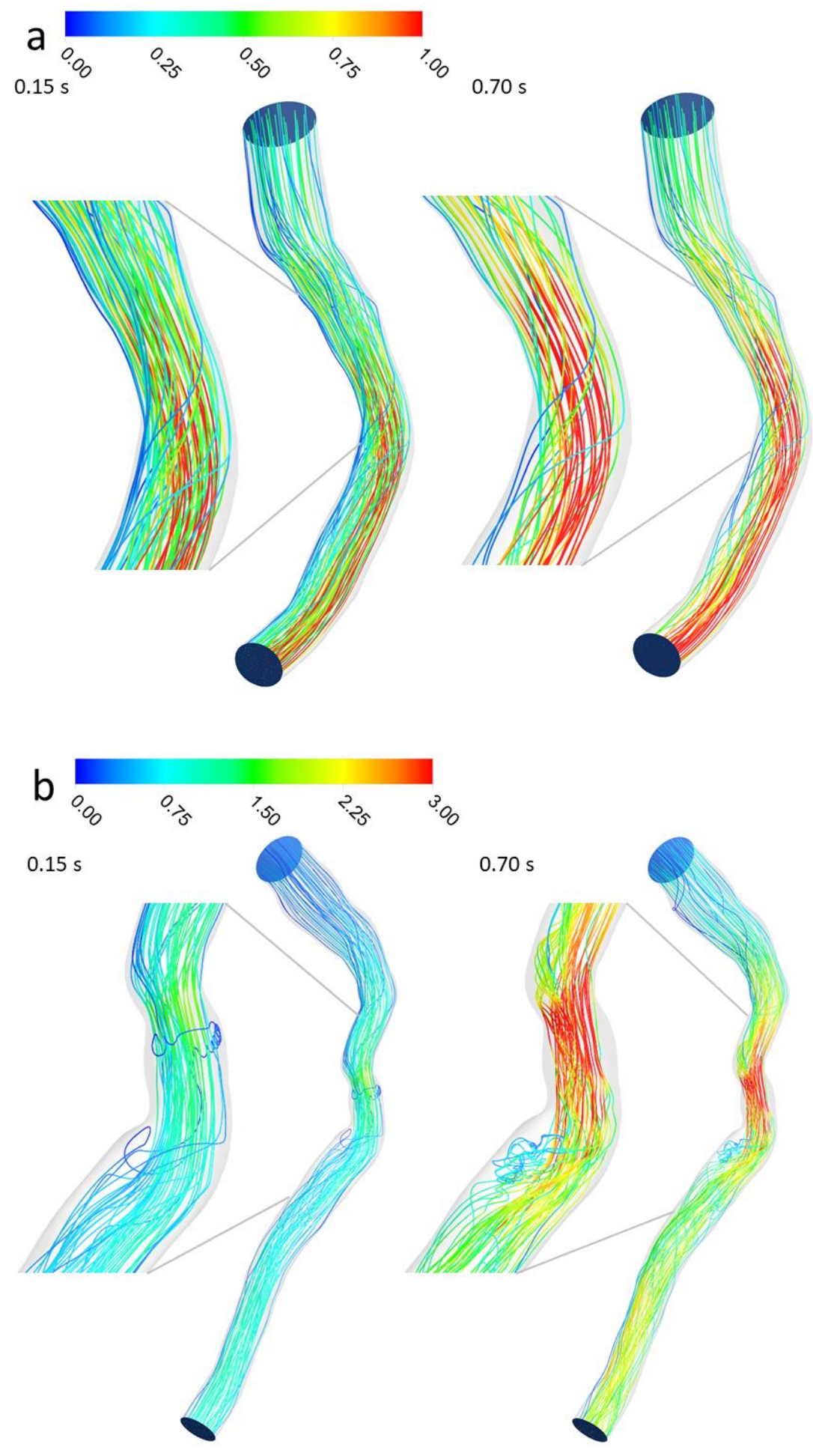

Figure 4.1 Blood flow pathlines (m/s) (a) for patient A and (b) for patient B during both systole ( $0.15 \mathrm{~s}$ of pulse) and diastole ( $0.7 \mathrm{~s}$ of pulse). 


\subsection{Wall Shear Stress}

Patient B has an elongated stenosis with high WSS throughout the stenosed region, but with a noticeable region of low WSS corresponding to the area of recirculation (Figure 4.2). WSS is generally more ordered with little variability for Patient A. Both images are during systole, at $0.7 \mathrm{~s}$ of the pulse.

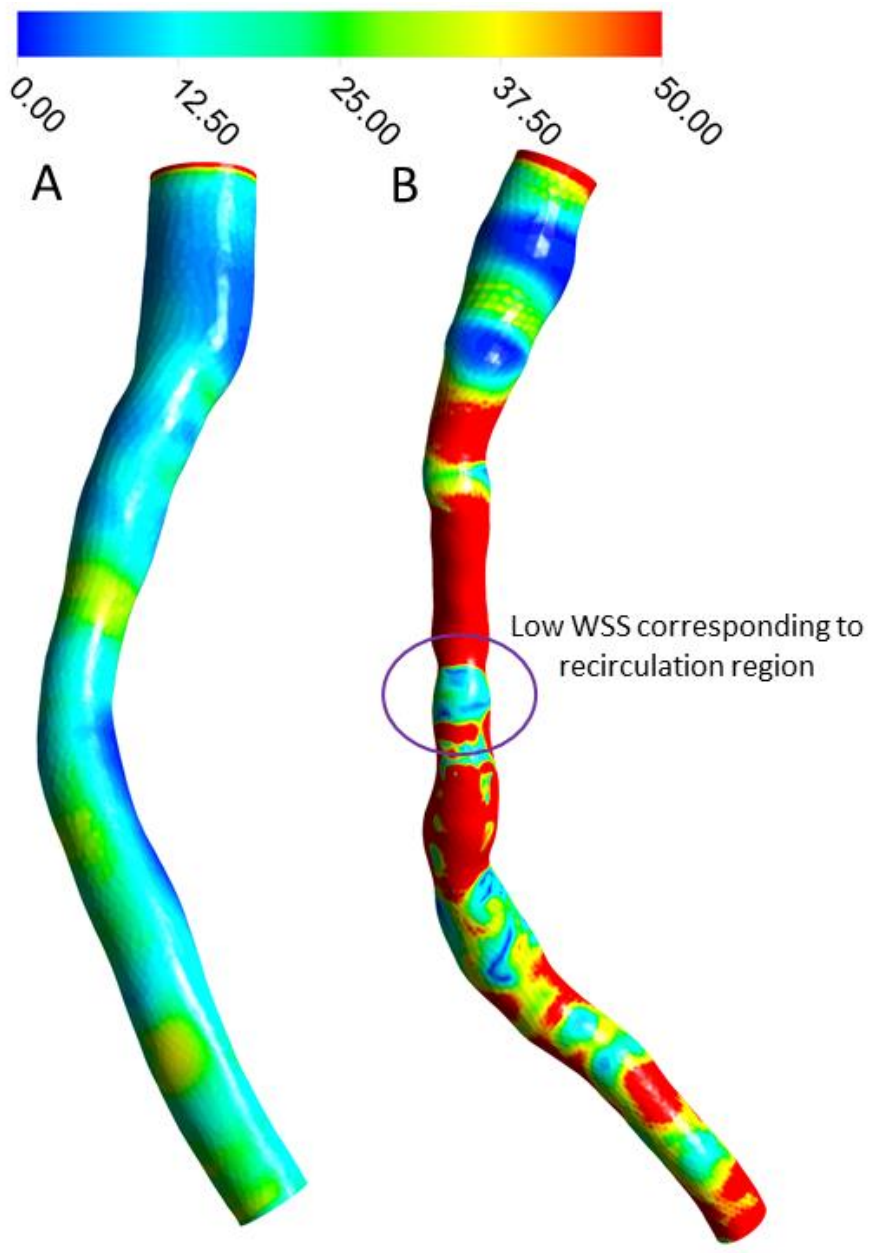

Figure 4.2 Wall shear stress $(\mathrm{Pa})$ in two sample patients. A low WSS region is noticeable where recirculation occurs exiting the elongated stenosis of Patient B. 


\subsection{Mean Residence Time}

Figure 4.3 shows pathlines colored by mean residence time for Patients A and B. The color indicates a methodical increase in mean residence time from inlet to outlet for Patient A since there is no real holdup in this patient's LAD. The overall mean residence time was $0.0817 \mathrm{~s}$, just $22 \%$ above its nominal mean residence time of $0.0670 \mathrm{~s}$, where nominal mean residence time is defined as volume divided by flow rate and represents the mean residence time that would be expected if flow was completely uninhibited. However, for Patient B, mean residence time in the recirculation region distal to stenosis is clearly high relative to the fluid passing in the main jet stream. The bulk of the mean time here is on the order of $50 \%$ higher than the main jet stream, with certain points are as much as three to four times higher. The overall mean time for this patient is $0.0796 \mathrm{~s}$, while its nominal mean time was $0.0535 \mathrm{~s}$, an increase of $49 \%$.

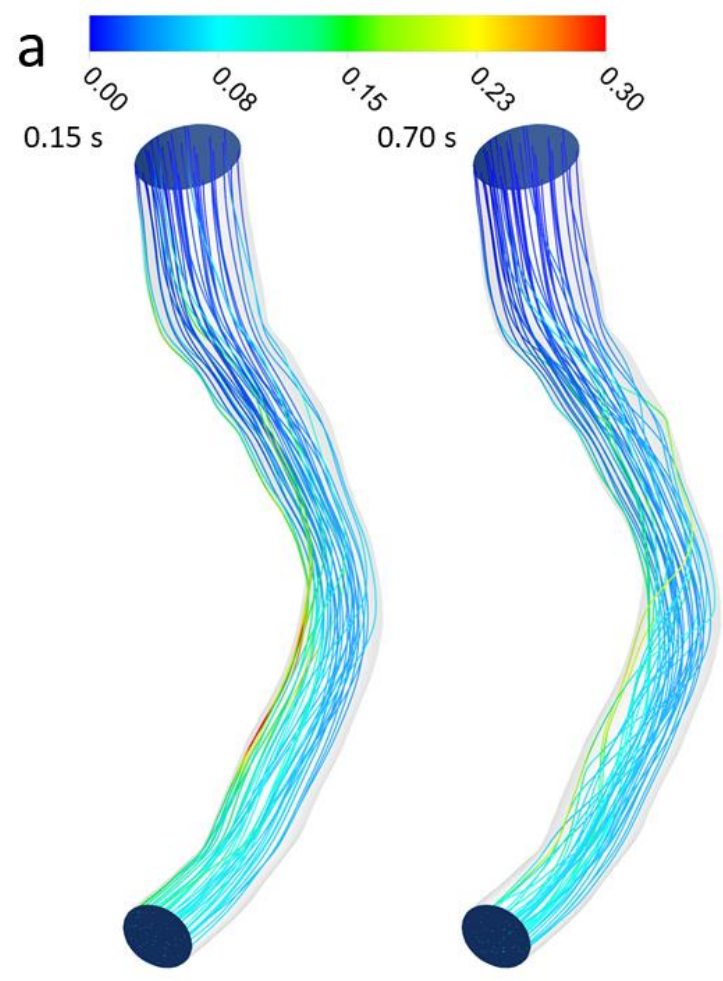




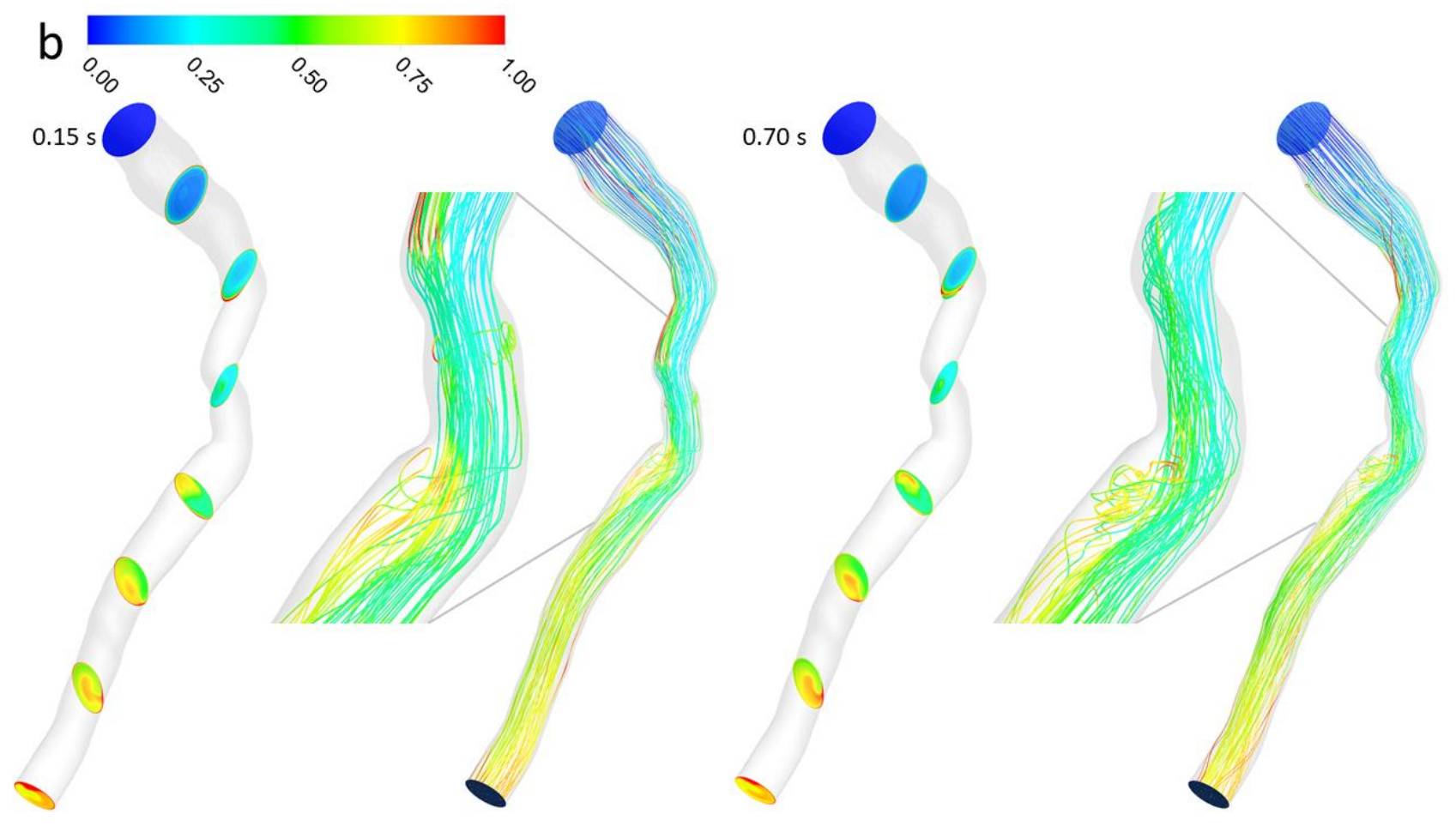

Figure 4.3 Mean residence time (s) pathlines (a) for patient A and (b) for patient B in systolic and diastolic phase.

Under normal conditions, mean residence time should increase during the systolic phase, when the velocity is generally lower, and decrease in the diastolic phase, when the velocity is generally higher. Mean residence time for patient A over the course of an entire pulse (Figure 4.4a) reflects this, where the amplitudes are low when velocity amplitudes are high and vice-versa. Also, as the slope of the velocity increases, the slope of mean time decreases and vice-versa. Mean time adheres to this during systole for Patient B, but more or less levels off during diastole when it should be decreasing (Figure 4.4b), which is reflective of the recirculation and holdup during the diastolic phase. The time in Figures $4.4 \mathrm{a}$ and $4.4 \mathrm{~b}$ represents the mean exit residence time of blood that entered the arterial segment at a given time during the cardiac cycle, and the overall mean value is reported as the average of mean residence times over one complete cycle. 
The pressure outlet boundary condition did not affect mean residence time for patient A (Figure 4.4a) or Patient B (Figure 4.4b). Figure 5a shows mean residence time throughout one pulse for the original pressure, half the original pressure, and zero (gauge) pressure for patient A. Mean residence time for these three examples were $0.0818 \pm 0.00001 \mathrm{~s}$. Figure $4.4 \mathrm{~b}$ shows the same for patient B with a mean residence time of $0.0796 \pm 0.00009 \mathrm{~s}$.
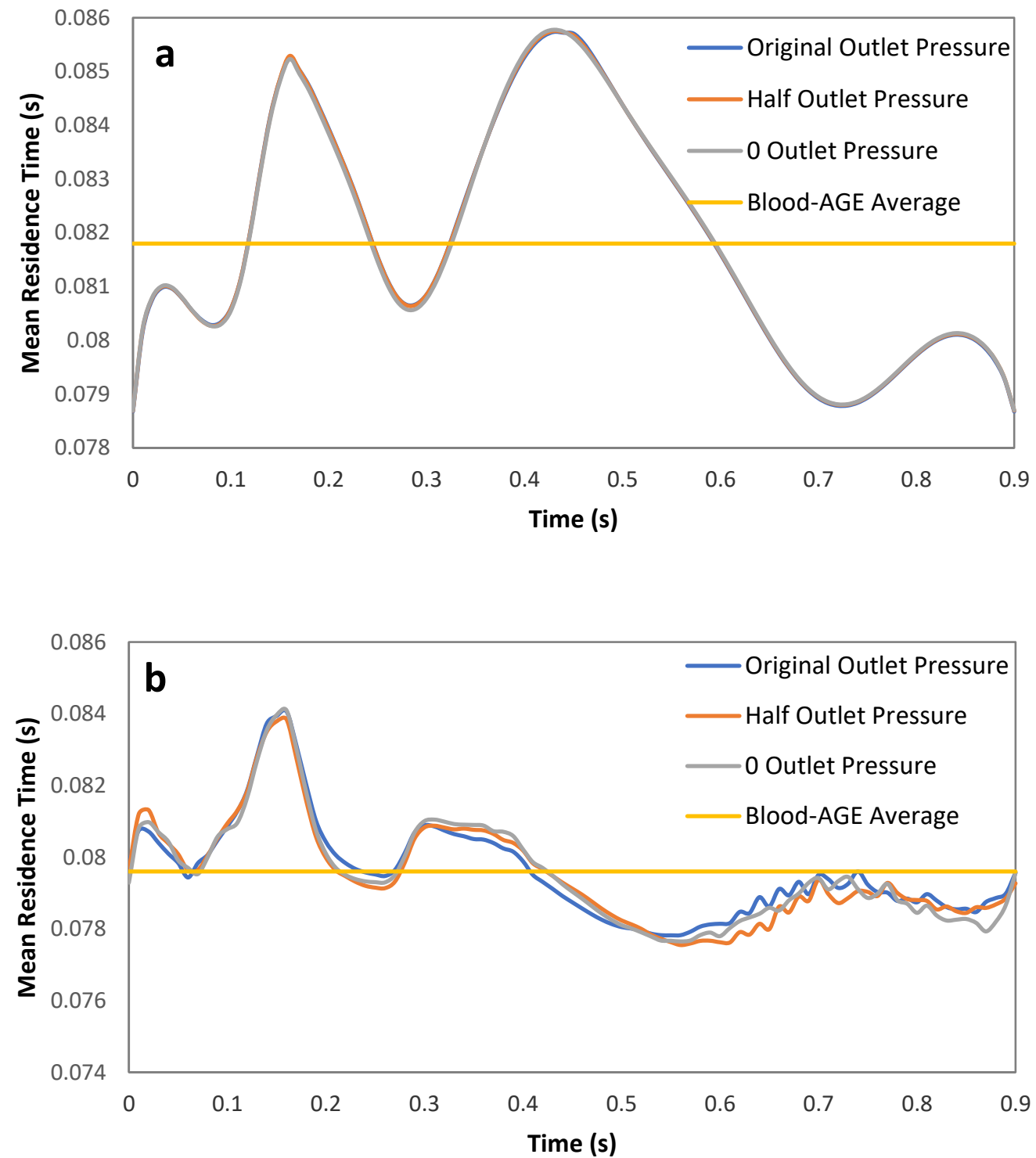

Figure 4.4 Mean residence time throughout one cardiac pulse for original outlet pressure, half the original outlet pressure, and 0 outlet pressure. (a) Patient A, (b) Patient B. 


\subsection{Blood $_{\mathrm{RT}}$ and Threshold}

Blood $_{\mathrm{RT}}$ is defined as a dimensionless parameter to account for varying length and volume of each arterial segment plus varying blood flow rates.

$$
\text { Blood }_{R T}=\left(\frac{\text { Nominal Mean Residence Time }(s)}{\text { Mean Residence Time }(s)}\right)
$$

Mean residence time was first determined in 100 coronary arteries for which the gold standard pressure-wire FFR was known. Abnormal $(\mathrm{FFR} \leq 0.80)$ and normal $(\mathrm{FFR}>0.80)$ groups based on the pressure-wire FFR threshold are highly associated with a Blood $\mathrm{RT}_{\mathrm{T}}$ threshold of 0.80 . There were 46 true negatives (46\%), 51 true positives (51\%), 1 false negative (1\%) and, 2 false positives (2\%) (Figure 4.5a). The sensitivity and specificity (along their $95 \%$ confidence intervals) are 98\% (88-100) and 96\% (86-100) respectively, indicating strong ability for Blood $_{\mathrm{RT}}$ to predict whether FFR is above or below 0.80. These AUC, sensitivity, and specificity values compared favorably to various forms of virtual FFR (Table 4.1). The ROC curve is presented in Figure $4.5 \mathrm{~b}$. While the main objective was to determine the Blood $\mathrm{RT}$ threshold for diagnostic accuracy, there was also a strong correlation between pressure-wire

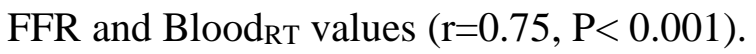

Table 4.1 Statistical analysis comparison between Blood $_{\mathrm{RT}}$ and various forms of virtual FFR.

\begin{tabular}{|l|l|l|l|l|}
\hline Metrics & Case Numbers & AUC & Sensitivity & Specificity \\
\hline Blood $_{\text {RT }}$ (this work) & 100 & 0.996 & 96 & 98 \\
\hline FFR $_{\text {angio }}$ [55] & 184 & 0.97 & 88 & 95 \\
\hline QFR [54] & 87 & 0.91 & 78 & 89 \\
\hline FFR & & & & \\
\hline
\end{tabular}




\begin{tabular}{|l|l|l|l|l|}
\hline vFAI [14] & 139 & 0.92 & 90.4 & 86.2 \\
\hline Virtual FFR- & 35 & & 71 & 100 \\
VIRTU-1 [53] & & & 93 & 85 \\
\hline $\begin{array}{l}\text { Stenosis flow } \\
\text { reserve (SFR) [91] }\end{array}$ & 110 & & & \\
\hline
\end{tabular}

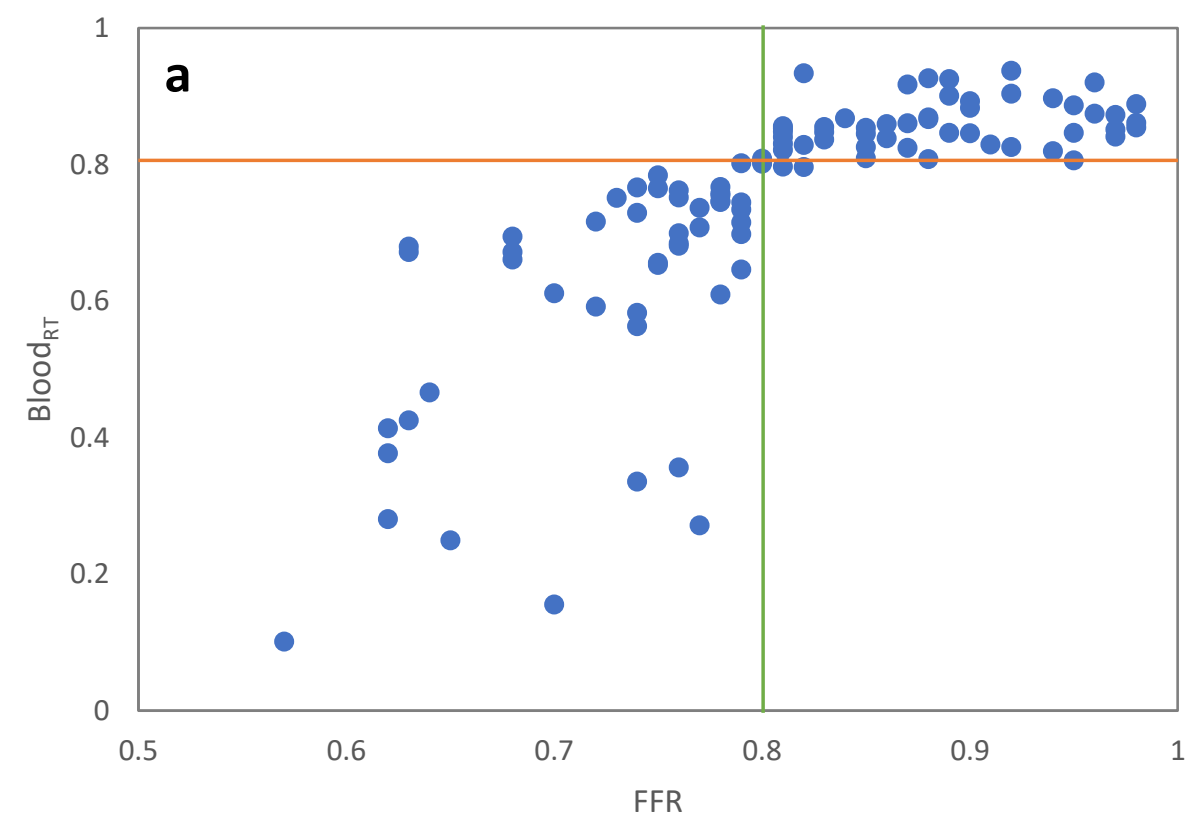




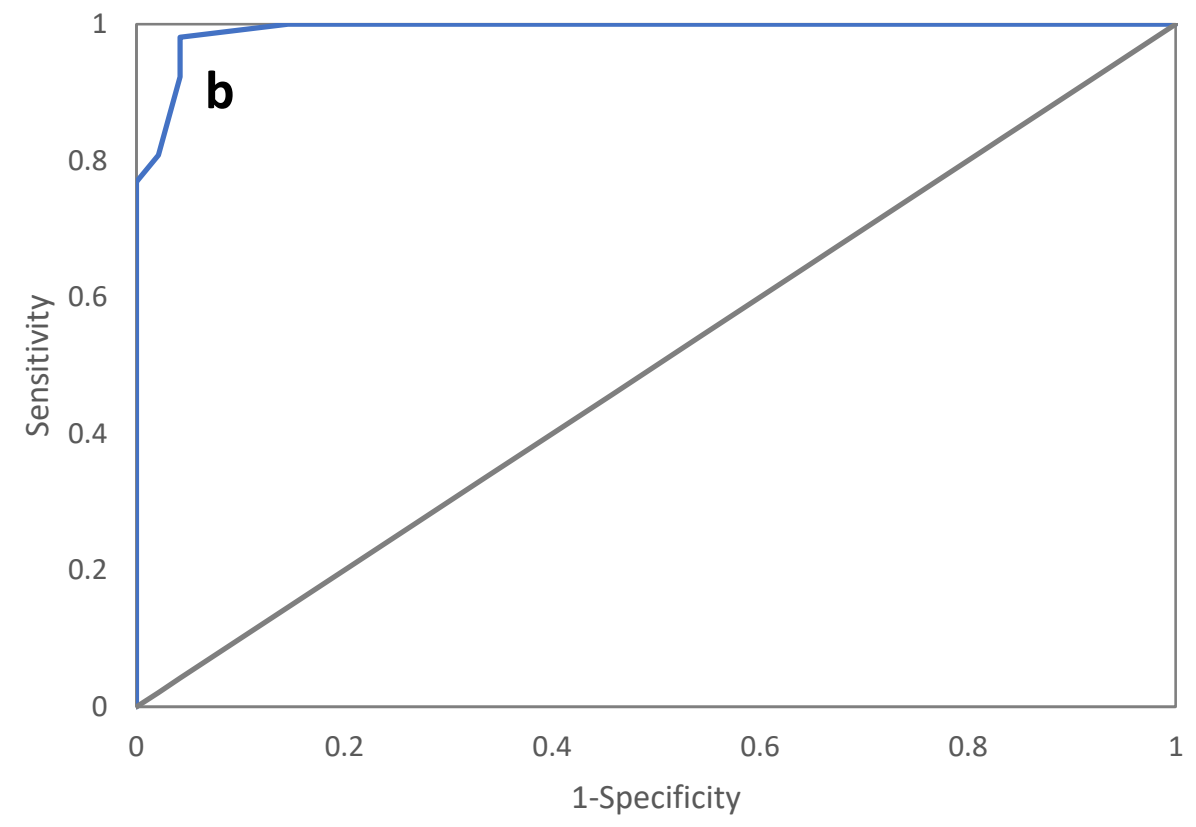

Figure 4.5 (a) Blood $\mathrm{RT}_{\mathrm{T}}$ and invasive FFR for 100 patient coronary arteries and (b) Receiver operator characteristic (ROC) curve analysis for Blood RT $_{\text {against the fractional flow reserve }}$ (area under curve $=0.9959)$.

\subsection{Discussion}

Pressure-wire FFR, the gold standard for diagnosing the physiological significance of coronary stenosis, is a function of pressure loss across the stenotic segment. Pressure loss is a characterization of the energy loss in the blood flow resulting from stenosis. The altered, disordered flow due to stenosis leads to frictional loss between layers of fluid, fluid and the wall, and especially around bends, through constrictions, and in observed recirculation regions, resulting in loss of pressure [92]. Rather than measuring or computing pressure loss to quantify physiological significance, we have presented a new approach which quantifies altered flow trajectories through the residence time metric, arguably a more direct measure of altered blood flow due to stenosis. 
While mean age theory has primarily been demonstrated in industrial systems $[60,63$, $67,93]$, it can also be useful for characterizing flow within vessels and human organs or their model counterparts. In this study, we employed mean age theory to characterize blood flow characteristics in coronary segments. Parameters such as relative velocity and WSS are indicative of changes in flow characteristics, but by themselves do not necessarily correlate to physiologic significance in stenotic coronary arteries [29]. On the other hand, mean residence time, especially relative to unobstructed flow, is a widely used established indicator of variance in flow. Two objects with equal volume and flow may have vastly different flow characteristics and, hence, mean residence time if their geometries or, in this case, anatomies differ.

Stenotic flows, which have been well characterized in several studies, exhibit flow separation downstream of the stenosis characterized by a central jet stream and secondary flow near the wall, with a strong shear layer in between $[93,94]$. The deceleration of flow during diastole is responsible for the conditions that create the secondary flow reversal downstream of the stenosis. The flow separation depends on the upstream flow velocity and diameter of the stenosis [95]. The velocity gradient and shear layer at the interface provide the potential for reversed flow due to the tangential force [96]. This effect occurred here just past the region of stenosis (Figure 4.1b).

Mean residence time increased relative to nominal mean time due to flow characteristics distal to the stenosis zone, with practically no effect on residence time proximal to the stenosis. Even a small fraction of blood held up while recirculating in the secondary flow region will cause the overall residence time to increase above the nominal value. Higher mean time in the recirculation region associated with Patient $\mathrm{B}$ was on the order of $1.5-4 \mathrm{X}$ the surrounding fluid that passes uninhibited, contributing to the overall increase in mean time at 
the exit or, by definition, decrease in the dimensionless Blood ${ }_{\mathrm{RT}}$. Blood ${ }_{\mathrm{RT}}$ for patient B's LAD with $\mathrm{FFR}=0.63$, was 0.67 . Both values indicate an extreme departure from their respective thresholds and are representative of severely disturbed flow due to an elongated stenosis.

While the recirculation pattern generally remains over time, fluid that enters this region eventually crosses back into the primary flow stream at the boundary between the primary and secondary streams. Else, if even a small amount of fluid were held up there indefinitely, mean residence time would approach infinity. The hold-up time and variance from nominal residence time depends on the combination and interactions of factors such as velocity through the stenosed area, the size of the stenosis, and shape of the artery segment such as if it is straight or bends.

The threshold between a hemodynamically significant or non-significant stenosis was also determined for our new Blood ${ }_{R T}$ metric. Blood ${ }_{R T}$ agreed with pressure-wire FFR in all but three cases on the hemodynamic significance of the stenosis. It is noteworthy that the noncompliant cases were within $\sim 0.5 \%$ of the statistically determined threshold; the Blood $\mathrm{RT}_{\mathrm{T}}$ of the two false positives were 0.796 and 0.797 , and the Blood ${ }_{\mathrm{RT}}$ of the false negative was 0.802 . Both the Blood $_{\mathrm{RT}}$ and pressure-wire FFR thresholds equal to a dimensionless value of $\sim 0.80$. Blood $_{\mathrm{RT}}$ is a measure of relative time while pressure-wire FFR is a measure of relative pressure. The two are indirectly related through fluid flow phenomena, but there is no reason other than coincidence that the two should be equal. It is possible that the Blood $\mathrm{RT}_{\mathrm{T}}$ threshold shifts if more cases are added to the study, but given the strong statistical correlation, any shift would likely be minimal. The similarity in thresholds does not imply that values should correlate for individual cases as Blood $\mathrm{RT}_{\mathrm{T}}$ is a measure of time while FFR one of pressure, however there 
was a close correlation between Blood $_{\mathrm{RT}}$ and pressure-wire FFR ( $\left.\mathrm{r}=0.753, \mathrm{p}<0.0001\right)$. Patient B provides a sound example with $\mathrm{FFR}=0.63$ and $\mathrm{Blood}_{\mathrm{RT}}=0.67$.

There was a region of decreased WSS (Figure 4.2) coinciding with the region of recirculation, which also coincided with increased mean residence time (Figure 4.3). Previously, Himburg et al. [78] introduced the concept of RRT, which is calculated from WSS and OSI rather than tracer measurements. Guerciotti et al. [97] also reported an inverse relation between WSS and RRT due to disturbed flow. However, they also stated that RRT provides no direct information on the actual residence time of blood in a given region. While use of the "relative residence time" is in some way an indicator of disturbed flow, it is actually a misnomer as it is reported with units of inverse pressure. The dimensionless parameter Blood $_{\mathrm{RT}}$, on the other hand, is a true direct measure of residence time. 


\section{CHAPTER FIVE}

\section{ASSESSMENT OF SIGNIFICANT STENOSIS FOR CORONARY ARTERY WITH}

\section{SERIAL STENOSES}

\subsection{Pathlines}

Figure 5.1 shows velocity pathlines in systolic $(0.15 \mathrm{~s})$ and diastolic $(0.7 \mathrm{~s})$ phase for patient $\mathrm{A}$ and $\mathrm{B}$ and $\mathrm{C}$, which all have serial stenoses and significant overall FFR values of $0.75,0.68$ and 0.78 , respectively. Overall FFR is measured between a point near the beginning of the aorta and distal to the last stenosis. In general, the pathlines remained orderly and did not exhibit holdup or recirculation distal to stenosis during the systolic phase because blood passed through the arteries with low flow rate. Some holdup and recirculation were seen during the diastolic phase when blood flow rate was increased. For patient $\mathrm{A}$ in diastolic phase, the velocity was uninterrupted proximal to the first stenosis and increased in magnitude in the throat of the first stenosis by 50-100\% (Figure 5.1a). After the first stenosis, flow separated into a high velocity stream and a low velocity region with recirculation. This low velocity holdup region stretched about half way to the second stenosis. A similar but smaller holdup region is visible after the second stenosis. These regions are quantified in Table 5.1 with Blood $_{\mathrm{RT}}$ values of 0.75 and 0.86 , where the lower Blood $\mathrm{RT}_{\mathrm{T}}$ corresponding to the $1^{\text {st }}$ stenosis is indicative of more pronounced holdup.

Velocity pathlines for patient B (Figure 5.1b) showed minimal regions of flow disturbance after each stenosis, and were correspondingly quantified with relatively high 
Blood $_{\mathrm{RT}}$ values of $0.88,0.90$, and 0.87 . For patient $\mathrm{C}$ (Figure 5.1c), flow separation and disturbance were not significant after the $1^{\text {st }}$ or $3^{\text {rd }}$ stenoses, but was more significant after the $2^{\text {nd }}$ stenosis. The corresponding Blood ${ }_{\mathrm{RT}}$ values were $0.97,0.84,0.93$.
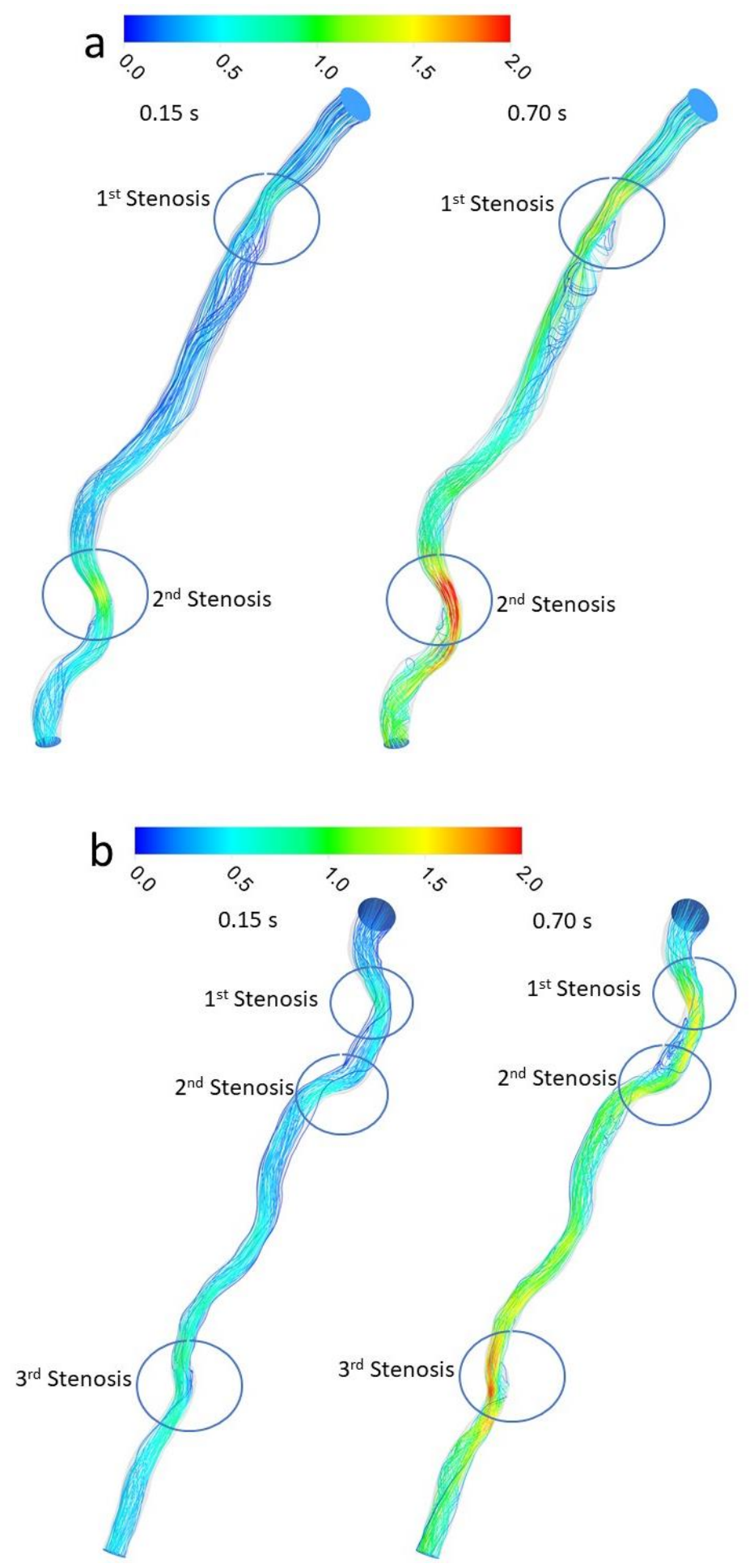


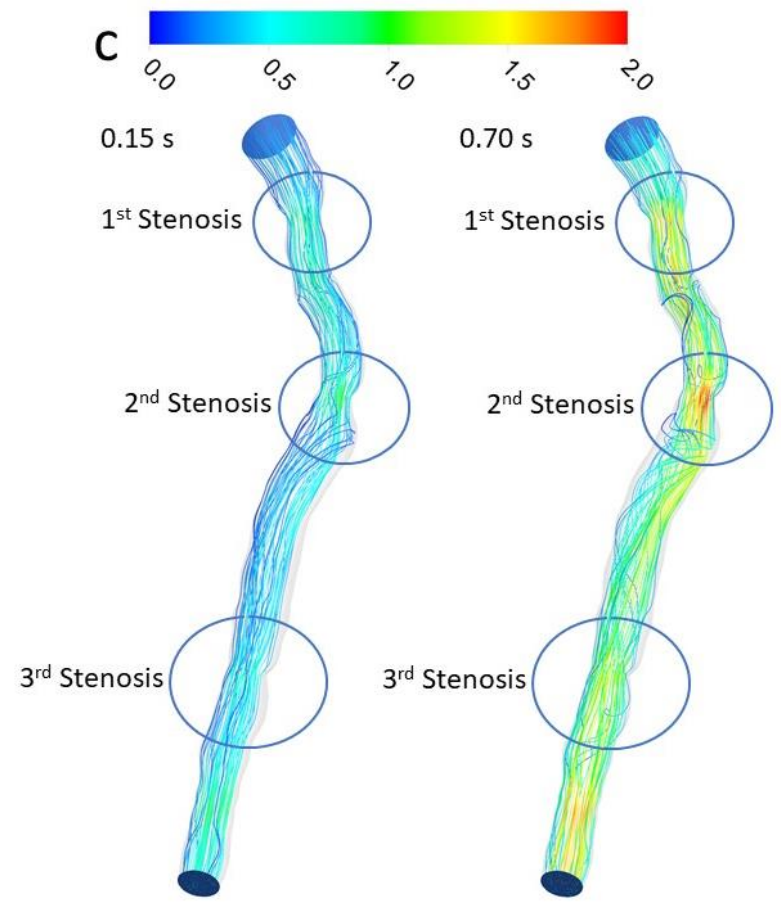

Figure 5.1 Blood velocity (m/s) pathlines for (a) Patient A, (b) Patient B and (c) Patient C.

\subsection{FFR}

Figure 5.2 shows vFFR contours traversed along the length of the coronary arteries. vFFR for each patient was 1.0 prior to any stenosis since there would not be any significant pressuere loss. Patient A's vFFR was reduced after each stenosis to 0.896 and 0.75 , respectively. vFFR remains virtually constant throughout unobstructed stretches and drops immediately at the point of stenosis. For patient B, vFFR was 0.891 after the first stenosis, 0.792 by the second stenosis, and 0.68 after third stenosis. For Patient C, vFFR after each stenosis was $0.924,0.873$, and 0.78 , respectively. The aforementioned values were overall FFR, meaning relative to the inlet of the artery. "Local" vFFR's are shown in Figure 5.2, which define an "inlet" as a point midway between the prior and upcoming stenoses. Local vFFR demonstrates that while one stenosis by itself, with a value well above 0.80 , may be 
insignificant, the combined contribution of serial stenoses can lead to overall FFR below the hemodynamically significant threshold. For example, FFR for Patient A dropped to 0.75 after the $2^{\text {nd }}$ stenosis even though the local FFR of the $2^{\text {nd }}$ stenosis was 0.934 .

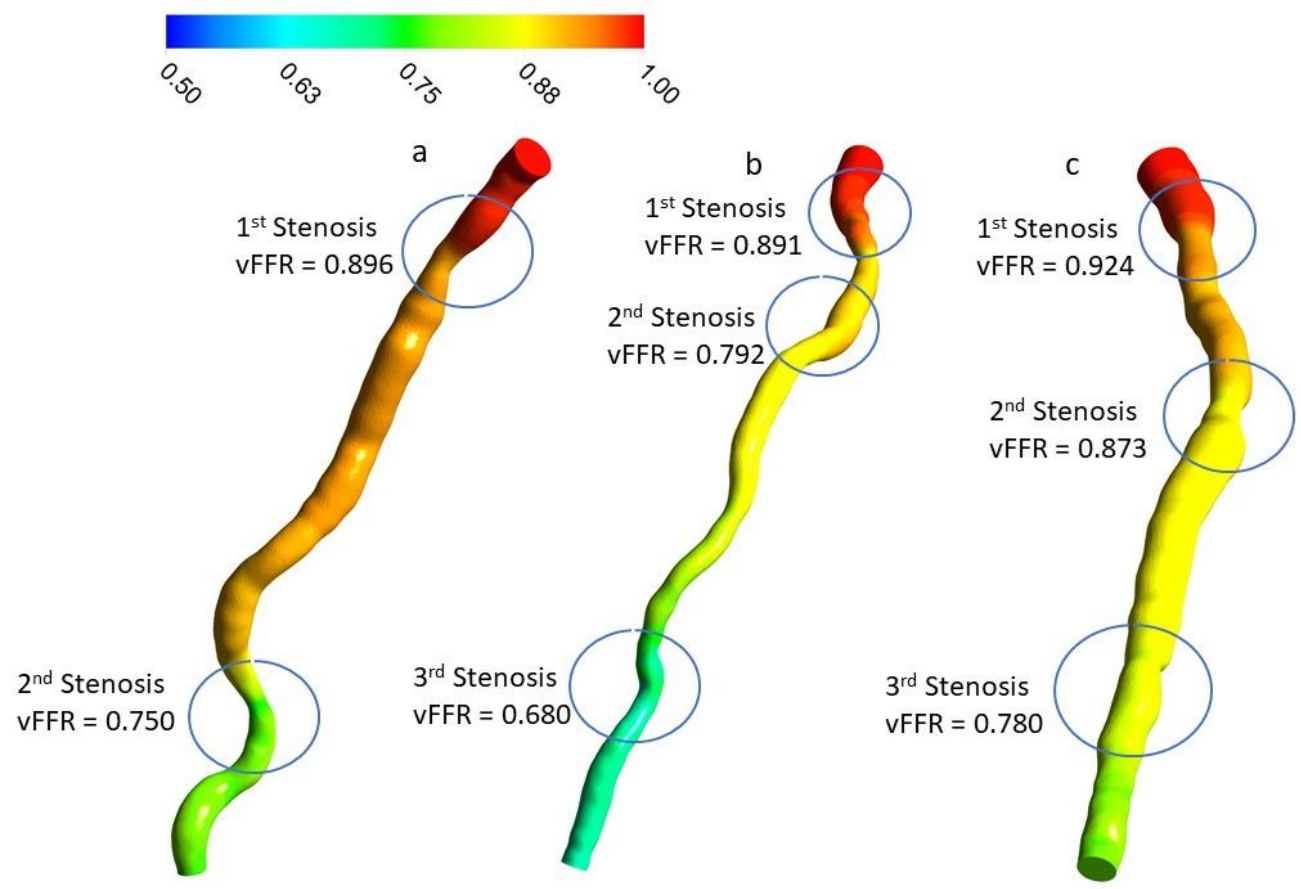

Figure 5.2 Localized FFR wall contours for Patient A, B and C.

\section{$5.3 \operatorname{Blood}_{\mathrm{RT}}$}

Similar to the concept of local FFR, local Blood ${ }_{\mathrm{RT}}$ 's were determined for each stenosis in a patient with serial stenosis. Figure 5.3 shows blood residence time along the length of an arterial segment for the three patients in both systolic and diastolic phase. Nominal and mean residence times were computed for segments indicated by the blue dashed lines. Planar slices are drawn at given intervals in addition to the longitudinal images. Local and overall Blood $\mathrm{RT}_{\mathrm{T}} \mathrm{s}$ are quantified along with local and overall FFR in Table 5.1. Patient $\mathrm{C}$ exhibits the best example 
of how each individual stenosis may be well above the threshold for significance, but the cumulative effect of all three stenosis leads to an overall hemodynamic significance; Blood $_{\mathrm{RT}}$ for the three stenosis are all above 0.9 , but the overall Blood $_{\mathrm{RT}}$ is 0.77 , which is below the threshold for significance.

a
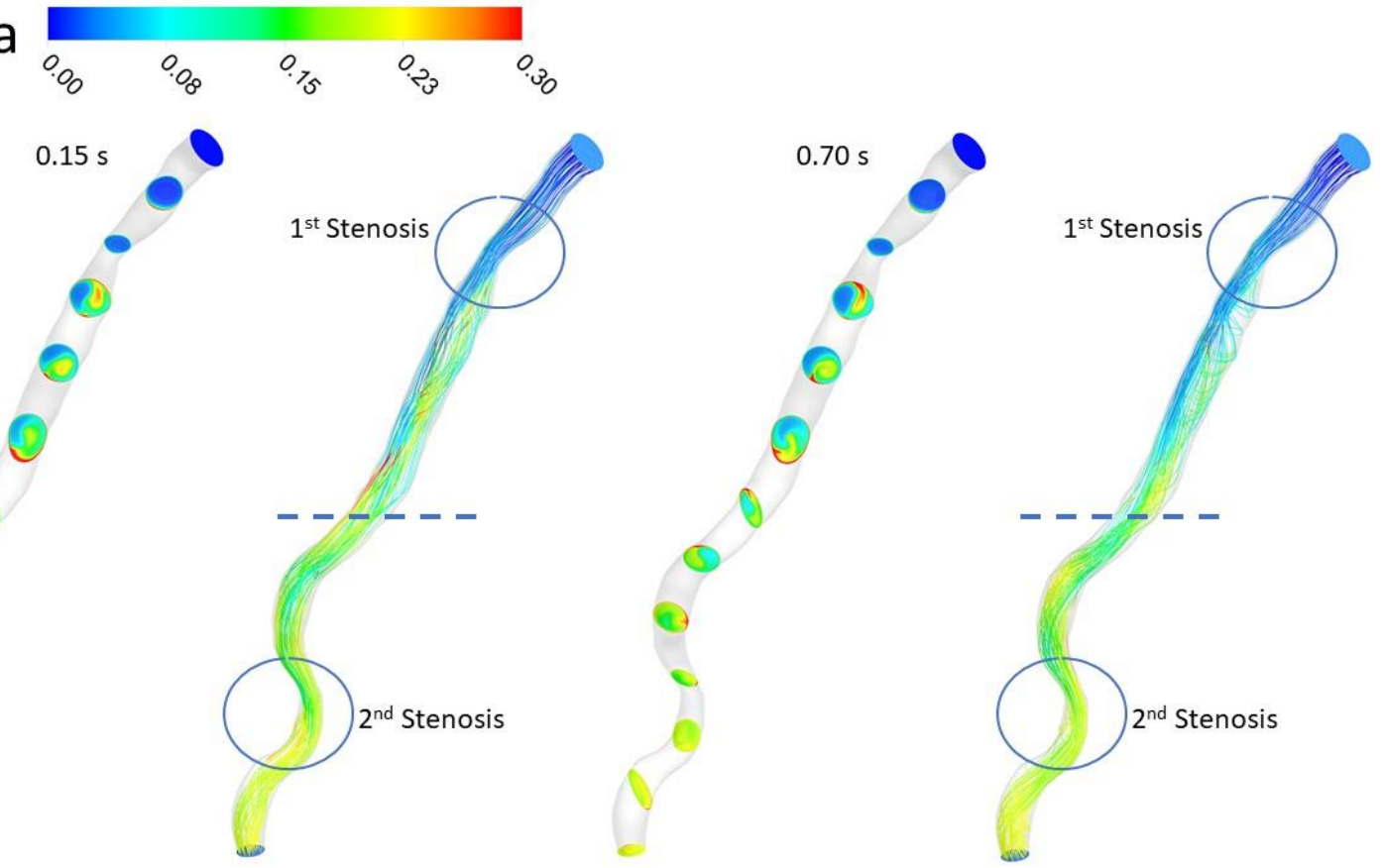

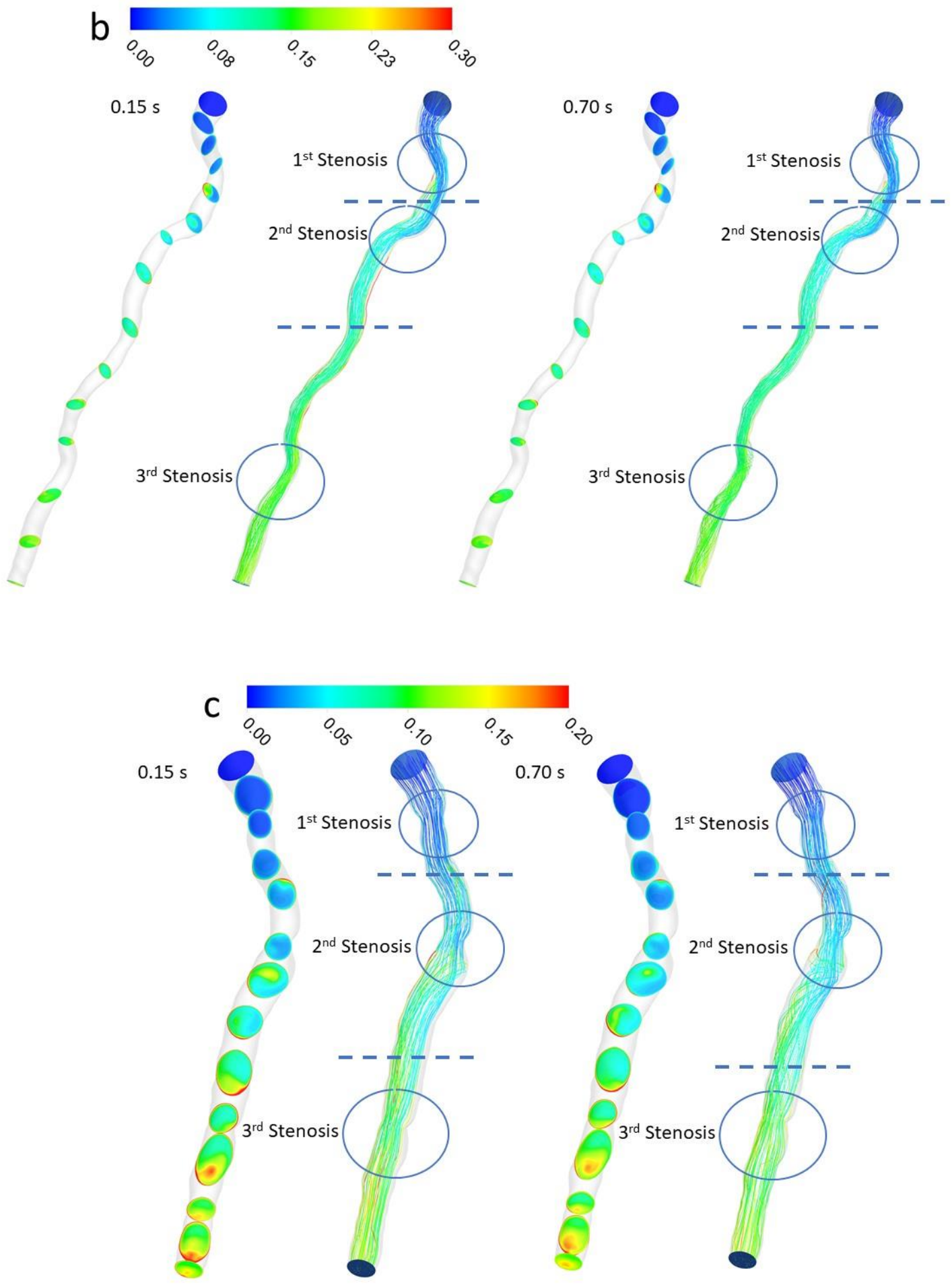

Figure 5.3 Slices of blood residence time (s) contours and pathlines along the arterial segment for (a) Patient A, (b) Patient B and (c) Patient C. 
Table 5.1 Local and Total Blood RT $_{\text {and }}$ FFR for each patient and stenosis.

\begin{tabular}{|c|c|c|c|c|c|c|c|c|c|}
\hline & \multicolumn{2}{|c|}{ Stenosis $1^{\text {st }}$} & \multicolumn{2}{|c|}{ Stenosis $2^{\text {nd }}$} & \multicolumn{2}{|c|}{ Stenosis $3^{\text {rd }}$} & \multicolumn{3}{|l|}{ Total } \\
\hline & Blood $_{\mathrm{RT}}$ & vFFR & Blood $_{\mathrm{RT}}$ & vFFR & Blood $_{\mathrm{RT}}$ & vFFR & Blood $_{\mathrm{RT}}$ & vFFR & FFR \\
\hline Patient A & 0.751 & 0.896 & 0.863 & 0.851 & & & 0.671 & 0.75 & 0.75 \\
\hline Patient B & 0.877 & 0.891 & 0.929 & 0.896 & 0.874 & 0.865 & 0.736 & 0.68 & 0.68 \\
\hline Patient C & 0.969 & 0.924 & 0.844 & 0.919 & 0.927 & 0.922 & 0.772 & 0.78 & 0.78 \\
\hline
\end{tabular}

The concept of local Blood $\mathrm{RT}$ is visualized in Figure 5.4, which shows one coronary artery circuit with serial stenosis and one with a single stenosis. $\mathrm{T}$ is Blood $_{\mathrm{RT}}$ of each stenosis, $\mathrm{t}$ is the nominal residence time of a segment, $\mathrm{C}$ is blood flow capacity, $\mathrm{Q}$ is flow rate, and $\mathrm{P}$ is pressure.

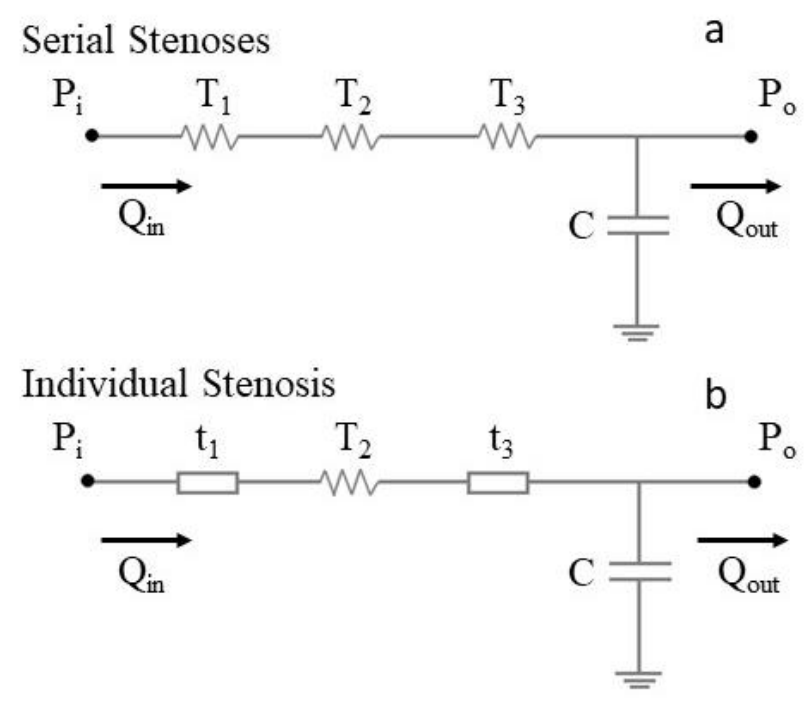

Figure 5.4 Stenosed coronary artery circuit with (a) serial stenoses and (b) individual stenosis. 


\subsection{Wall Shear Stresses}

Figure 5.5 shows blood WSS contours in systolic and diastolic phase for all three patients. For all three patients, WSS was generally higher in the diastolic phase than in the systolic phase because of higher velocity. WSS is high (red regions) in the throats of stenoses due to the high velocity jets streams and lower in the low velocity holdup regions.

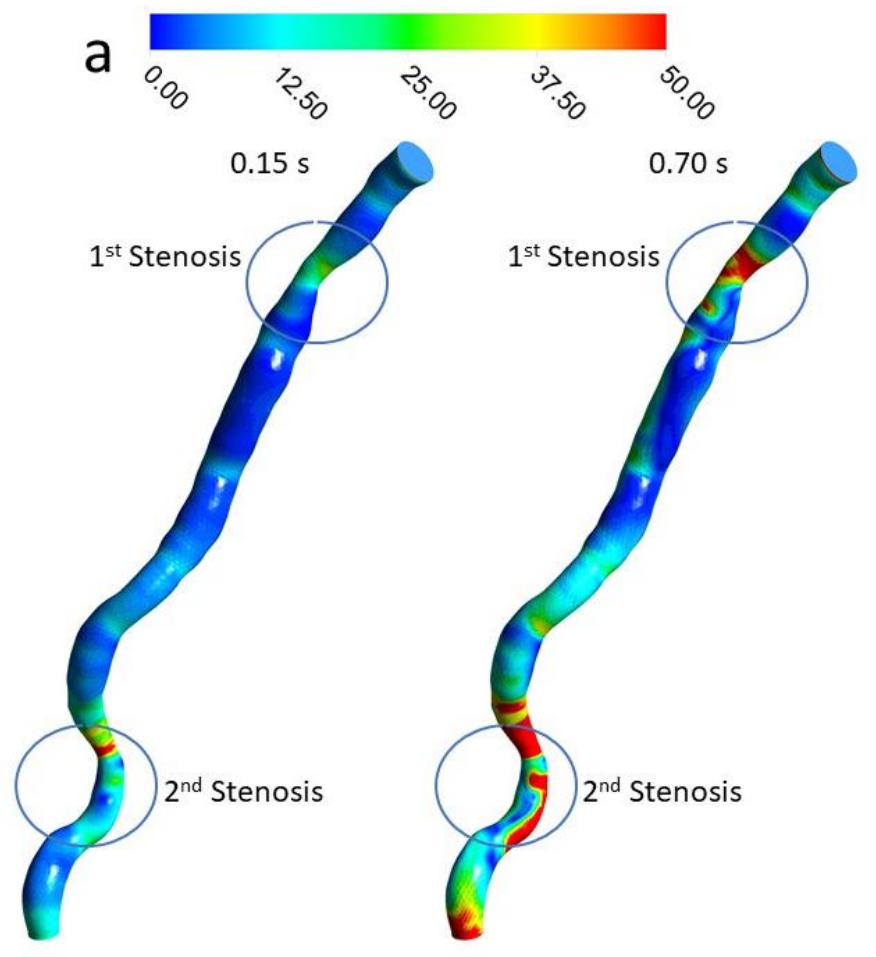



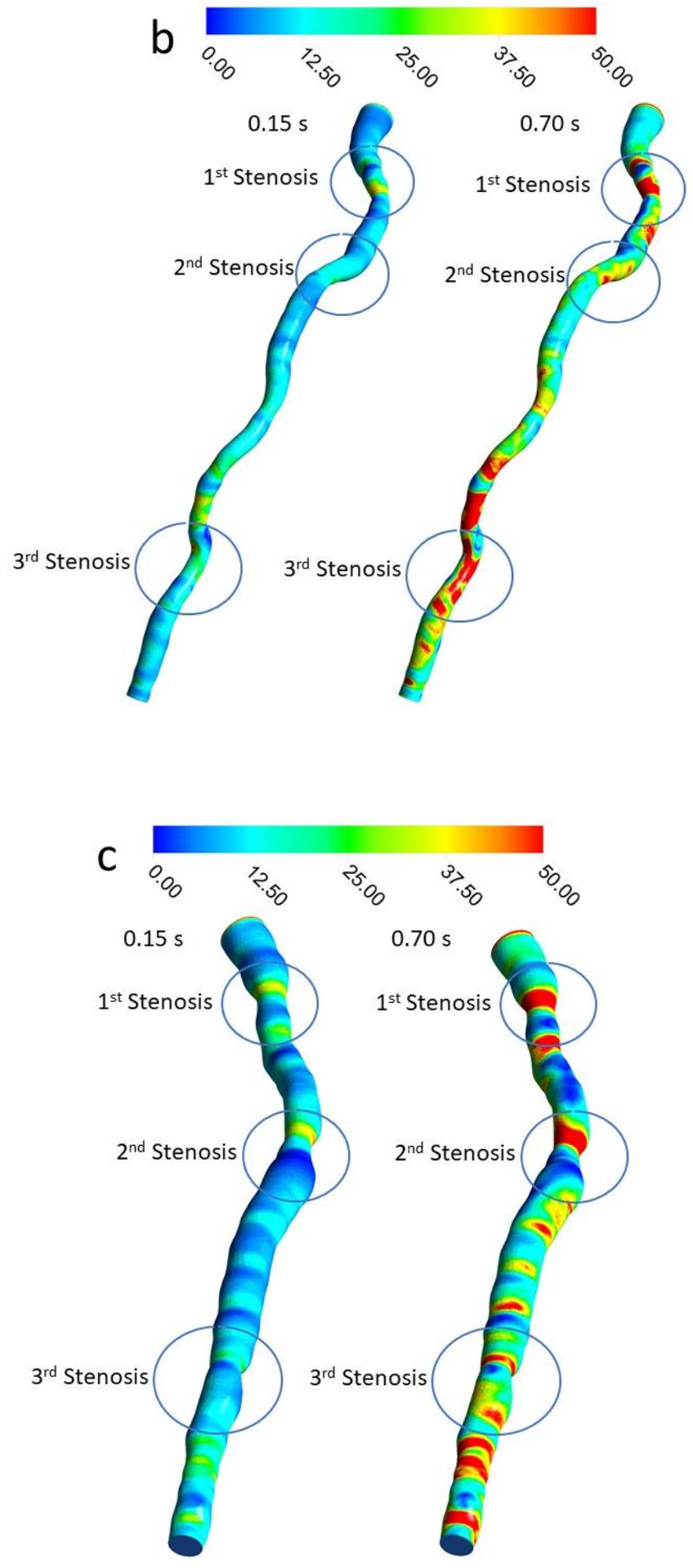

Figure 5.5 Blood WSS (Pa) contours on the wall for (a) Patient A, (b) Patient B and (c) Patient C. 


\subsection{Discussion}

Atherosclerotic plaque development depends on different factors like blood flow, blood cells, cholesterol concentration, and arterial geometry. Some patients with coronary artery disease have some serial stenosis along an artery. Decision making for treatment and determining FFR is complex [8]. FFR depends on pressure drop, which depends on factors such as stenosis severity, volume flow rate, and proximal pressure for each stenosis $[8,9]$. Determining FFR for each individual stenosis within serial stenoses is difficult to do clinically [8]. vFFR could identify significant stenosis better than invasive FFR, but it is not perfect because pressure proximal for each stenosis is affected by each previous stenosis [10].

CFD was employed here to study the flow characteristics in coronary arteries with serial stenosis for three patients. Flow disturbance following a stenosis could recover prior to the next stenosis in the series, meaning flow becomes ordered again. However, pressure did not necessarily recover. For patient A, inlet pressure measured at the coronary artery was $12000 \mathrm{~Pa}$, which then reduced to $10750 \mathrm{~Pa}$ after the first stenosis, which then became the proximal pressure for the second stenosis. Individual FFR depended on distal pressure of the previous stenosis. Understanding the contribution of each individual stenosis is important for making decisions about stent size and location. CFD is well suited for determining overall FFR as well as individual contributions of each stenosis in series to the overall FFR, where this is difficult to do clinically. Local and overall Blood RT $_{\text {were }}$ also computed for each stenosis.

Based on the three patients studied, a series of hemodynamically insignificant stenoses

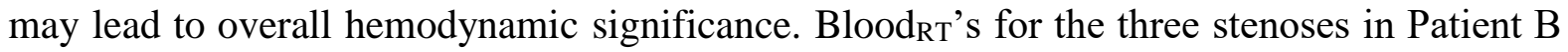
were all between $0.87-0.89$ (each were insignificant based on the previously determined threshold), but the overall Blood RT $_{\text {wa }}$ 0.736, which is considered hemodynamically 
significant. Similarly for Patient $\mathrm{C}$, all three stenosis had individual Blood $_{\mathrm{RT}}$ values between 0.844 and 0.969 , but the overall Blood $\mathrm{RT}_{\mathrm{T}}$ was 0.772 , which is much smaller than any individual value. Overall Blood ${ }_{\mathrm{RT}}$ for Patient A, which had a first stenosis Blood $\mathrm{RT}$ equal to 0.751, dropped to 0.671 following a second stenosis Blood $\mathrm{RT}$ equal to 0.863 .

Serial Blood $R T$ characteristics mirrored vFFR in the same patients. Patient B had local vFFR values between 0.865 to 0.896 , but the overall invasive and vFFR were both 0.68 . Local vFFR's for Patient $C$ were between 0.919 and 0.924 , all extremely close to the 1.0 value indicative of a healthy artery, but the overall FFR was 0.78 . Patient A, with a first stenosis FFR equal to 0.896 , dropped to an overall FFR equal to 0.75 despite a second stenosis with a high FFR equal to 0.851 .

Based on the three patients studied here, Blood $\mathrm{RT}_{\mathrm{T}}$ is at least equally sensitive to $\mathrm{vFFR}$ in assessing the overall significance of stenosis in series. While Blood ${ }_{\mathrm{RT}}$ gave fewer false readings than vFFR for single stenosis, this study on serial stenosis would need to be expanded significantly to determine if one is superior to the other.

WSS contours (Figure 5.5) supported velocity and residence time characteristics. WSS decreased in regions where velocity decreased and residence time increased due to holdup and recirculation. Also, WSS increased in the throat of stenoses where velocity is high and residence time is low. WSS, by definition, is more closely related to velocity and hence residence time than to pressure. WSS may be able to provide another means for assessing significance of a stenosis. Previous studies showed a correlation between WSS and a parameter they called relative residence time [78], which was not a direct measure of residence time, but a relative measure computed from WSS and a previously defined oscillatory shear index. 


\section{CHAPTER SIX}

\section{VOLUME FLOW RATE DETERMINATION USING MULTIPLE LINEAR}

\section{REGRESSION}

\subsection{Blood Flow Rate Determination}

A multiple linear regression approach was employed to determine coronary volume flow rate for patients undergoing coronary angiography. The focus was on two main components of the process: (i) feature extraction and selection; and (ii) ensemble learning algorithms. The training data collection consists of a matrix $\mathbf{X}$ and a vector $\mathbf{y}$. Here, $\mathbf{X}$ is an $n$ $\mathrm{x} p$ matrix that represents the $p$ descriptors (i.e., factors) of the $n$ patients, and $\mathbf{y}$ is an $n$ dimensional vector that represents the measurement (blood volume flow) to be predicted. The main goal was to use multiple linear regression and statistical data analysis to learn a mapping from $\mathbf{X}$ to $\mathbf{y}$. This mapping can then be used to predict the blood volume flow rate of a new patient that was not included in the training data $\mathbf{X}$.

The actual inlet blood volume flow rate proximal to stenotic coronary segments was determined from clinical data as described in Chapter 3. The available factors affecting inlet blood volume flow rate are: Coronary segment type (A), inlet diameter of the segment (B), stenosis diameter (C), stenosis percentage (D), inlet area of the segment (E), and stenosis area (F). CFD modeling suggests that the most crucial factor in determination of inlet blood volume

flow rate is segment type (factor A). The clustering method was used to divide coronary arteries into proximal, mid, and distal segments. Then, multiple linear regression was used with other 
factors for each subgroup. Table 6.1 shows the model expression for flow rate and accuracy for each segment. Figure 6.1 demonstrates the accuracy of this method in predicting coronary inlet volume flow rate for each segment, with clinical based inlet blood volume flow rate as the reference.

Table 6.1 Regression model for blood flow rate clustered by segment type.

\begin{tabular}{|c|c|c|c|c|c|}
\hline $\begin{array}{l}\text { Segmen } \\
\text { t Type }\end{array}$ & $\begin{array}{l}\text { Patient } \\
\text { Number }\end{array}$ & $\begin{array}{l}\text { Inlet } \\
\text { Diameter } \\
\text { Range } \\
(\mathrm{mm})\end{array}$ & $\begin{array}{l}\text { Validation } \\
\mathrm{R}^{2}\end{array}$ & $\begin{array}{l}\text { Regression } \\
\text { Model } \mathrm{R}^{2}\end{array}$ & $\begin{array}{l}\text { Volume Flow Rate } \\
\text { Regression Model }\end{array}$ \\
\hline $\begin{array}{l}\text { Prox } \\
\text { RCA }\end{array}$ & 4 & $3.5-5$ & 0.989 & 1 & $\begin{array}{l}=1.474 \mathrm{E}-005- \\
1.890 \mathrm{E}- \\
007 * \mathrm{C} * \mathrm{D}+1.247 \mathrm{E}- \\
009 * \mathrm{D} * \mathrm{E}+2.590 \mathrm{E}- \\
007 * \mathrm{~F}^{\wedge} 2\end{array}$ \\
\hline $\begin{array}{l}\text { Mid } \\
\text { RCA }\end{array}$ & 6 & $2.8-3.7$ & 0.974 & 0.997 & $\begin{array}{l}=-5.465 \mathrm{E}-005 \\
+9.855 \mathrm{E}- \\
007 * \mathrm{D}+5.808 \mathrm{E}- \\
006 * \mathrm{C}^{\wedge} 2-3.009 \mathrm{E}- \\
007 * \mathrm{E}^{\wedge} 2+2.960 \mathrm{E}- \\
006 * \mathrm{~F}^{\wedge} 2\end{array}$ \\
\hline $\begin{array}{l}\text { Dist } \\
\text { RCA }\end{array}$ & 4 & $3.8-4$ & 0.986 & 1 & $\begin{array}{l}=3.529 \mathrm{E}-006- \\
3.576 \mathrm{E}- \\
006 * \mathrm{C}+1.217 \mathrm{E}-\end{array}$ \\
\hline
\end{tabular}




\begin{tabular}{|c|c|c|c|c|c|}
\hline & & & & & $\begin{array}{l}008 * \mathrm{D}+5.318 \mathrm{E}- \\
007 * \mathrm{~F}^{\wedge} 2\end{array}$ \\
\hline $\begin{array}{l}\text { Prox } \\
\text { LAD }\end{array}$ & 21 & $3.5-5$ & 0.942 & 0.954 & $\begin{array}{l}=-4.975 \mathrm{E}-005 \\
+1.495 \mathrm{E}-006 * \mathrm{D}- \\
3.680 \mathrm{E}-006 * \mathrm{E} \\
+1.509 \mathrm{E}- \\
007 * \mathrm{~B} * \mathrm{D}-1.331 \mathrm{E}- \\
006 * \mathrm{C} * \mathrm{D} \\
+5.040 \mathrm{E}- \\
007 * \mathrm{D}^{*} \mathrm{~F}-9.890 \mathrm{E}- \\
007 * \mathrm{E}^{*} \mathrm{~F}-2.680 \mathrm{E}- \\
006 * \mathrm{~B}^{\wedge} 2+2.853 \mathrm{E}- \\
005^{*} \mathrm{C}^{\wedge} 2+3.069 \mathrm{E}- \\
007 * \mathrm{E}^{\wedge} 2-3.078 \mathrm{E}- \\
006^{*} \mathrm{~F}^{\wedge} 2\end{array}$ \\
\hline $\begin{array}{l}\text { Mid } \\
\text { LAD }\end{array}$ & 42 & $2-4.5$ & 0.852 & 0.82 & $\begin{array}{l}=-1.548 \mathrm{E}- \\
005+2.857 \mathrm{E}- \\
005 * \mathrm{C}+2.798 \mathrm{E}- \\
007 * \mathrm{D}+5.730 \mathrm{E}- \\
007 * \mathrm{E}+2.504 \mathrm{E}- \\
006 * \mathrm{~F}-1.522 \mathrm{E}- \\
007 * \mathrm{~B} * \mathrm{D}-1.617 \mathrm{E}- \\
007 * \mathrm{C} * \mathrm{D}+7.556 \mathrm{E}-\end{array}$ \\
\hline
\end{tabular}




\begin{tabular}{|c|c|c|c|c|c|}
\hline & & & & & $\begin{array}{l}007 * \mathrm{C}^{*} \mathrm{E}-4.781 \mathrm{E}- \\
008^{*} \mathrm{D}^{*} \mathrm{~F}+6.764 \mathrm{E}- \\
008^{*} \mathrm{E} * \mathrm{~F}+2.100 \mathrm{E}- \\
006^{*} \mathrm{~B}^{\wedge} 2-1.450 \mathrm{E}- \\
005^{*} \mathrm{C}^{\wedge} 2-7.612 \mathrm{E}- \\
008^{*} \mathrm{E}^{\wedge} 2+1.048 \mathrm{E}- \\
007 * \mathrm{~F}^{\wedge} 2\end{array}$ \\
\hline $\begin{array}{l}\text { Dist } \\
\text { LAD }\end{array}$ & 3 & $2.8-3.8$ & 0.933 & 0.905 & $\begin{array}{l}=2.226 \mathrm{E}- \\
006+2.303 \mathrm{E}- \\
008^{*} \mathrm{C} * \mathrm{D}+1.533 \mathrm{E}- \\
009^{*} \mathrm{D} * \mathrm{E}-1.322 \mathrm{E}- \\
008^{*} \mathrm{D}^{*} \mathrm{~F}-6.302 \mathrm{E}- \\
010^{*} \mathrm{D}^{\wedge} 2-4.095 \mathrm{E}- \\
008^{*} \mathrm{~F}^{\wedge} 2\end{array}$ \\
\hline $\begin{array}{l}\text { Prox } \\
\text { LCX }\end{array}$ & 7 & $2.8-4$ & 0.992 & 1 & $\begin{array}{l}=1.156 \mathrm{E}-005- \\
8.360 \mathrm{E}- \\
008 * \mathrm{D}+4.890 \mathrm{E}- \\
007 * \mathrm{E}+6.593 \mathrm{E}- \\
008 * \mathrm{~B} * \mathrm{D}-2.534 \mathrm{E}- \\
007 * \mathrm{C} * \mathrm{D}-1.002 \mathrm{E} \\
008 * \mathrm{D}^{*} \mathrm{~F}+1.637 \mathrm{E}- \\
007 * \mathrm{~F}^{\wedge} 2\end{array}$ \\
\hline
\end{tabular}




\begin{tabular}{|c|c|c|c|c|c|}
\hline $\begin{array}{l}\text { MID } \\
\text { LCX }\end{array}$ & 13 & $3.1-4$ & 0.981 & 1 & $\begin{array}{l}=3.079 \mathrm{E}-005- \\
4.427 \mathrm{E}- \\
005 * \mathrm{~B}+1.874 \mathrm{E}- \\
007 * \mathrm{~B} * \mathrm{D}+1.059 \mathrm{E}- \\
006^{*} \mathrm{C} * \mathrm{D}-1.511 \mathrm{E}- \\
007 * \mathrm{D} * \mathrm{~F}+2.673 \mathrm{E}- \\
006 \mathrm{~F}^{\wedge} 2\end{array}$ \\
\hline $\begin{array}{l}\text { Total } \\
\text { Patients }\end{array}$ & & 100 & & & \\
\hline
\end{tabular}

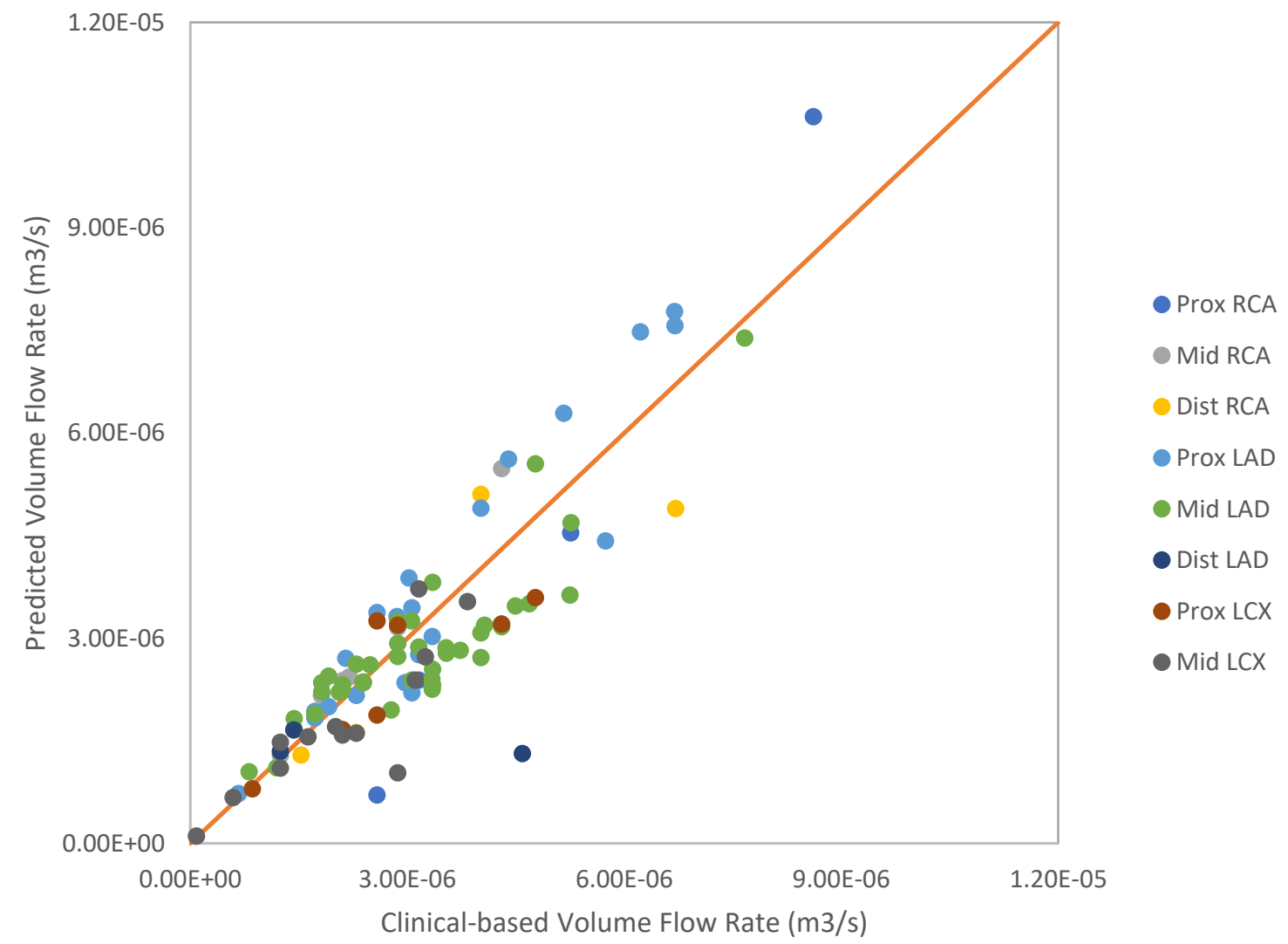

Figure 6.1 Predicted and clinical-based volume flow rate. 


\subsection{Blood $_{\mathrm{RT}}$ Computed with Predicted Coronary Blood Flow Rate}

In order to further validate this method, Blood RT $_{\text {was }}$ computed for a new set of 100 patients using the predicted blood volume flow rate as the inlet boundary condition. The Blood $_{\mathrm{RT}}$ threshold for hemodynamic significance was compared with that of the pressure wire measured FFR (Figure 6.2a). There were 34 true negatives (34\%), 62 true positives (62\%), 1 false positive ( $2 \%$ ) and 2 false negatives ( $2 \%$ ). Additionally, there were three instances of FFR

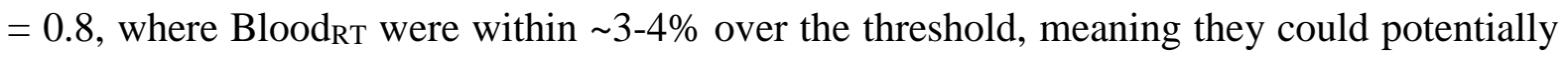
be considered as slight false negatives, although could also be considered as accurate given a reasonable assumption of measurement error. The sensitivity and specificity (along with their $95 \%$ confidence intervals) are $94.6 \%$ (88-100) and 96.8\% (86-100) respectively, indicating strong ability for Blood $\mathrm{RT}_{\mathrm{T}}$ to predict whether FFR is above or below 0.80 . The ROC curve is presented in Figure 6.2b. While the main objective was to determine the Blood $\mathrm{RT}_{\mathrm{T}}$ threshold for diagnostic accuracy, there was also a strong correlation between pressure-wire FFR and Blood $\left._{\text {RT values }(r=0.74, ~} \mathrm{P}<0.001\right)$. 

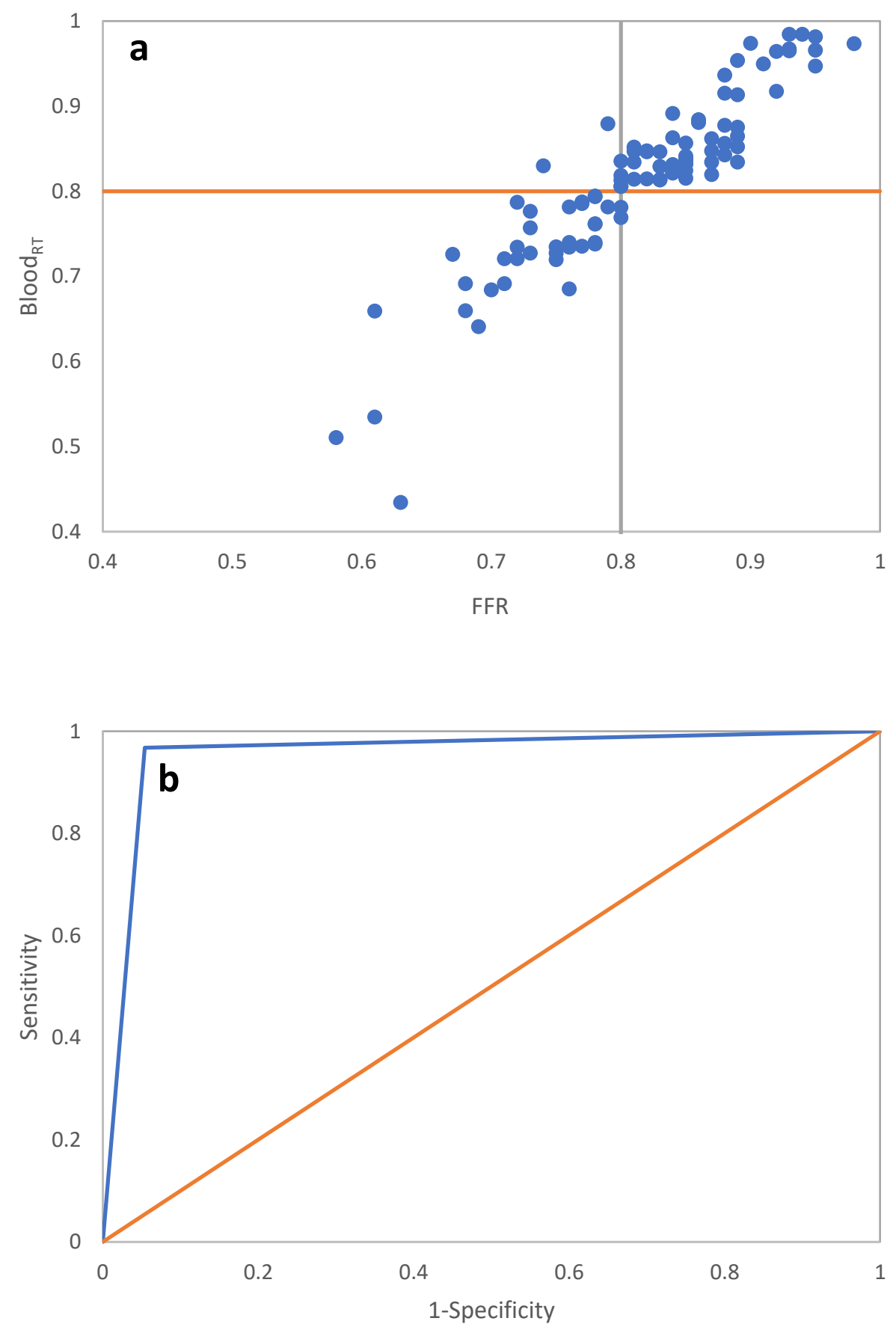

Figure 6.2 (a) Blood $\mathrm{RT}_{\mathrm{T}}$ and invasive FFR for 100 patient coronary arteries and (b) ROC curve analysis for Blood ${ }_{\mathrm{RT}}$ against the fractional flow reserve (area under curve $=0.957$ ). 


\subsection{Discussion}

One important challenge in angiographically calculated vFFR of coronary lesions is that the coronary inlet volume flow rate, which is the volume flow rate of blood proximal to coronary stenosis, is required as an inlet boundary condition to enable computation of vFFR without the need to model blood flow throughout the entire coronary tree. However, determining this inlet flow rate is problematic because it requires knowing the left ventricle volume, which is not obtainable from coronary angiography. Alternate approaches have been used for defining volume flow rate as a boundary condition. Papafaklis et al. [14] and Pellicano et al. [55] used a fix number of $1 \mathrm{ml} / \mathrm{min}$ or $3 \mathrm{ml} / \mathrm{min}$ for all patients depending on whether they modeled baseline or hyperemic conditions. This is not a realistic approach since every patient and each coronary artery segment type have different volume flow rates. Tu et al. [49] measured contrast medium transport time in the reconstructed vessel on hyperemic projections using TIMI frame count. The mean volume flow rate at hyperemia was then derived using the lumen volume of the reconstructed coronary tree divided by the mean transport time. This approach enables individualized quantification of inlet volume flow rate for patients compared to a "one-size-fits-all" strategy, but results showed that the method was not accurate for volume flow rate estimation $\left(\mathrm{r}^{2}=0.38\right)[49]$.

Results here show there is significant variation in inlet volume flow rate, mostly dependent on the segment type containing the stenosis. Multiple linear regression analysis was used here for the first time to determine volume flow rate in coronary arteries. First, factors that are readily available from coronary angiography rendering software were identified. The patients were divided into subgroups based on specific segment types (Table 6.1), and a regression was performed for each segment type. 
The same viscosity value was used for each patient in this 100 patient validation study.

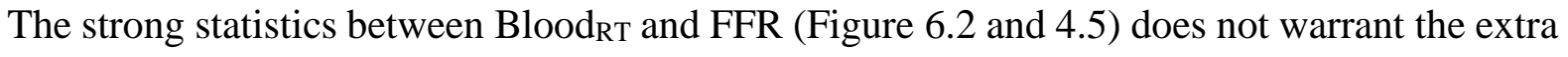
clinical measurement that would be required to obtain a personalized viscosity for each patient. The $\mathrm{R}^{2}$ for seven of the eight segment types was between 0.9 and 1.0. The Mid LAD regression model had the lowest accuracy (0.82), likely due to the widest variation of a key factor, the inlet diameter. The mid LAD diameters ranged from $2-4.5 \mathrm{~mm}$. The largest was $2.25 \mathrm{X}$ wider than the smallest. The inlet diameters of all other segments had a smaller range of variation; Prox RCA (3.5-5 mm), Mid RCA (2.8-3.7 mm), Dist RCA (3.8-4 mm), Prox LAD (3.5-5 mm), Dist LAD (2.8-3.8), Prox LCX (2.8-4) and MID LCX (3.1-4). The largest difference was 1.4X, with one as low as $1.05 \mathrm{X}$.

The statistical significance between Blood $_{\mathrm{RT}}$ and FFR (Figure 6.2) confirmed the accuracy of the regression derived volume flow rate. Further, the results obtained with the regression derived flow rate were statistically similar to the results obtained with the clinically derived flow rate (Figure 4.5). Specificity and sensitivity from the regression flow rate were with $1 \%$ of the clinical flow rate. The correlation of values was also very close, 0.74 for the regression flow and 0.75 for the clinical flow. The regression derived flow rate sometimes deviated as much as $30-40 \%$ from the clinical standard. However, one advantage of a dimensionless Blood $_{\mathrm{RT}}$ metric is that, while the flow rate does not directly cancel out, it is factored into both the nominal and CFD computed mean times, allowing room for error. In conclusion, based on sensitivity, specificity, and AUC statistical parameters, the regression model provided a valid method for determining volume flow rate, a key inlet boundary condition for non-invasively computing Blood $_{\mathrm{RT}}$. 


\section{CHAPTER SEVEN}

\section{CONCLUSION}

A new computational based metric was developed to assess the physiological significance of coronary stenosis without the use of invasive pressure-wire measurement. The

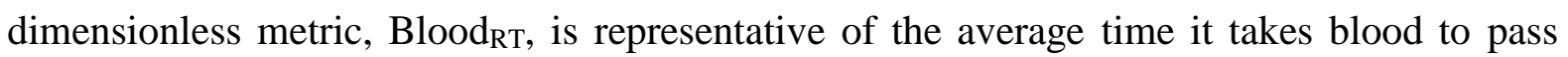
through a given arterial segment, and is indicative of the increase in time as compared to the nominal time, defined as volume divided by flow rate, for blood transit through that segment. Increase in mean residence time above the nominal time is due to a small region of recirculatory flow distal to stenosis as elucidated by model derived pathlines. The method incorporated established mean age theory for computational efficiency, although other methods for computing the mean time may be suitable. The method was then applied to 100 coronary arteries from patients who had already undergone the pressure-wire FFR measurement for clinical indications. A threshold for Blood RT $_{\text {was }}$ determined that demonstrates excellent discrimination in detecting significant from non-significant stenosis compared to the gold standard pressure-wire FFR.

Accurate and readily available boundary conditions for blood flow modeling in small artery segments are a common concern. Multiple linear regression was applied to estimate blood flow into each artery segment, providing a complete noninvasive method to replace the clinically determined blood flow that was used as a boundary condition to develop the model used in the first 100 patient study. A separate regression model was developed for each of eight 
artery segment types. Accuracy defined by $\mathrm{R}^{2}$ was between 0.9 and 1.0 for seven of the eight segment types, and 0.82 for the other one. A second 100 artery patient study was performed using the new blood flow boundary condition, and Blood ${ }_{\mathrm{RT}}$ again correlated very strongly statistically with the pressure-wire FFR. Specificity and sensitivity were 95\% and 97\%, comparable to the $96 \%$ and $98 \%$ from the first patient study.

Blood $_{R T}$ was then applied to artery segments containing multiple stenoses in series. The cumulative effect of all stenoses led to an overall Blood $_{R T}$ below the threshold of hemodynamic significance even when each individual stenosis was above the threshold. The overall Blood $_{\mathrm{RT}}$ determined the same outcome as pressure wire FFR for each of the three serial stenosis cases. 


\section{CHAPTER EIGHT RECOMMENDATIONS}

The first recommendation is to re-run the 100 patient study to confirm the Blood $_{R T}$ threshold using the Lattice Boltzmann CFD method rather than the finite volume method employed in this work with Ansys Fluent. Lattice Boltzmann is known to scale better with the number of processors, and can therefore, significantly reduce time processing. A single proof of concept case was run using Lattice Boltzmann based CFD software from MStar. Run time was on the order of minutes using a GPU processor, compared to several hours when run in parallel on 18 processors. A run time of less than five minutes is needed for this method to be suitable for use in a cardiac catheterization lab. The same blood properties and boundary conditions should be used in this confirmation study.

OSI is a widely used index to characterize flow, which can be used for studying artery disease. Further study may determine if a relationship exists between Blood ${ }_{R T}$ and OSI.

Blood flow in coronary arteries was predicted here using regression algorithms. The regression model may be improved by increasing the number of training cases, or possibly considering additional anatomical factors. Other factors such as age, sex, and body type could be explored as well.

The models were developed here from retrospective studies using patient data obtained from two local hospitals, the University of Louisville Hospital and Jewish Hospital. Larger 
scale prospective studies should be performed with patients from several more hospitals. An advantage of performing a prospective study is that cardiologists can be more attentive to the camera angles in order to improve the quality of the 3D renderings. 


\section{REFERENCES}

1. Benjamin, E.J., P. Muntner, and M.S. Bittencourt, Heart disease and stroke statistics2019 update: A report from the American Heart Association. Circulation, 2019. 139(10): p. e56-e528.

2. Boden, W.E., et al., Optimal medical therapy with or without PCI for stable coronary disease. New England journal of medicine, 2007. 356(15): p. 1503-1516.

3. Group, B.D.S., A randomized trial of therapies for type 2 diabetes and coronary artery disease. New England Journal of Medicine, 2009. 360(24): p. 2503-2515.

4. Tonino, P.A., et al., Fractional flow reserve versus angiography for guiding percutaneous coronary intervention. New England Journal of Medicine, 2009. 360(3): p. 213-224.

5. Tanaka, N., et al., Coronary flow-pressure relationship distal to epicardial stenosis. Circulation journal, 2003. 67(6): p. 525-529.

6. Nijjer, S.S., et al., Coronary pressure and flow relationships in humans: phasic analysis of normal and pathological vessels and the implications for stenosis assessment: a report from the Iberian-Dutch-English (IDEAL) collaborators. European heart journal, 2015. 37(26): p. 2069-2080.

7. Pijls, N.H., et al., Percutaneous coronary intervention of functionally nonsignificant stenosis: 5-year follow-up of the DEFER Study. Journal of the American College of Cardiology, 2007. 49(21): p. 2105-2111. 
8. Pijls, N.H., et al., Coronary pressure measurement to assess the hemodynamic significance of serial stenoses within one coronary artery: validation in humans. Circulation, 2000. 102(19): p. 2371-2377.

9. De Bruyne, B., et al., Pressure-derived fractional flow reserve to assess serial epicardial stenoses: theoretical basis and animal validation. Circulation, 2000. 101(15): p. 1840-1847.

10. Liu, X., et al., Hemodynamics analysis of the serial stenotic coronary arteries. Biomedical engineering online, 2017. 16(1): p. 127.

11. Taylor, C.A., T.A. Fonte, and J.K. Min, Computational fluid dynamics applied to cardiac computed tomography for noninvasive quantification of fractional flow reserve: scientific basis. Journal of the American College of Cardiology, 2013. 61(22): p. $2233-2241$.

12. Morris, P.D., et al., "Virtual"(computed) fractional flow reserve: current challenges and limitations. JACC: Cardiovascular Interventions, 2015. 8(8): p. 1009-1017.

13. Min, J.K., et al., Noninvasive fractional flow reserve derived from coronary $C T$ angiography: clinical data and scientific principles. JACC: Cardiovascular Imaging, 2015. 8(10): p. 1209-1222.

14. Papafaklis, M.I., et al., Fast virtual functional assessment of intermediate coronary lesions using routine angiographic data and blood flow simulation in humans: comparison with pressure wire-fractional flow reserve. EuroIntervention: journal of EuroPCR in collaboration with the Working Group on Interventional Cardiology of the European Society of Cardiology, 2014. 10(5): p. 574-583. 
15. Bhaskaran, R. and L. Collins, Introduction to CFD basics. Cornell University-Sibley School of Mechanical and Aerospace Engineering, 2002: p. 1-21.

16. Fluent, A., 17.0 Theory Guide, 2015, Canonsburg, PA 15317: ANSYS. Inc.

17. Steinman, D., D. Vorp, and C. Ethier, Computational modeling of arterial biomechanics: insights into pathogenesis and treatment of vascular disease. Journal of Vascular Surgery, 2003. 37(5): p. 1118-1128.

18. Qiu, Y. and J.M. Tarbell, Interaction between wall shear stress and circumferential strain affects endothelial cell biochemical production. Journal of vascular research, 2000. 37(3): p. 147-157.

19. Zeng, D., et al., Effects of cardiac motion on right coronary artery hemodynamics. Annals of biomedical engineering, 2003. 31(4): p. 420-429.

20. Steinman, D.A., Image-based computational fluid dynamics modeling in realistic arterial geometries. Annals of biomedical engineering, 2002. 30(4): p. 483-497.

21. Migliavacca, F., et al., A predictive study of the mechanical behaviour of coronary stents by computer modelling. Medical Engineering \& Physics, 2005. 27(1): p. 13-18.

22. Gidaspow, D., Multiphase flow and fluidization: continuum and kinetic theory descriptions. 1994: Academic press.

23. Leuprecht, A. and K. Perktold, Computer simulation of non-Newtonian effects on blood flow in large arteries. Computer Methods in Biomechanics and Biomedical Engineering, 2001. 4(2): p. 149-163.

24. Lightfoot, E. and T. Phenomena, Living Systems: Biomedical Aspects of Momentum and Mass Transport. 1974, Wiley, New York, NY. 
25. Bird, R.B., R.C. Armstrong, and O. Hassager, Dynamics of polymeric liquids. Vol. 1: Fluid mechanics. 1987.

26. Stuart, J. and M.W. Kenny, Blood rheology. Journal of clinical pathology, 1980. 33(5): p. 417.

27. Long, D.S., et al., Microviscometry reveals reduced blood viscosity and altered shear rate and shear stress profiles in microvessels after hemodilution. Proceedings of the National Academy of Sciences, 2004. 101(27): p. 10060-10065.

28. Waters, S.L., et al., Theoretical models for coronary vascular biomechanics: progress \& challenges. Progress in biophysics and molecular biology, 2011. 104(1-3): p. 49-76.

29. Zhang, J.M., et al., Hemodynamic analysis of patient-specific coronary artery tree. International journal for numerical methods in biomedical engineering, 2015. 31(4): $\mathrm{p}$. e02708.

30. Samady, H., et al., Coronary artery wall shear stress is associated with progression and transformation of atherosclerotic plaque and arterial remodeling in patients with coronary artery disease. Circulation, 2011. 124(7): p. 779-788.

31. Jin, S., et al. Flow patterns and wall shear stress distributions at atherosclerotic-prone sites in a human left coronary artery-an exploration using combined methods of CT and computational fluid dynamics. in The 26th Annual International Conference of the IEEE Engineering in Medicine and Biology Society. 2004. IEEE.

32. Ku, D.N., Blood flow in arteries. Annual review of fluid mechanics, 1997. 29(1): p. $399-434$. 
33. Park, S.-J., et al., Validation of functional state of coronary tandem lesions using computational flow dynamics. The American journal of cardiology, 2012. 110(11): p. 1578-1584.

34. Uren, N.G., et al., Relation between myocardial blood flow and the severity of coronary-artery stenosis. New England Journal of Medicine, 1994. 330(25): p. 17821788.

35. Gould, K.L. and K. Lipscomb, Effects of coronary stenoses on coronary flow reserve and resistance. The American journal of cardiology, 1974. 34(1): p. 48-55.

36. Gould, K.L., R.L. Kirkeeide, and M. Buchi, Coronary flow reserve as a physiologic measure of stenosis severity. Journal of the American College of Cardiology, 1990. 15(2): p. 459-474.

37. Pijls, N., et al., Experimental basis of determining maximum coronary, myocardial, and collateral blood flow by pressure measurements for assessing functional stenosis severity before and after percutaneous transluminal coronary angioplasty. Circulation, 1993. 87(4): p. 1354-1367.

38. Pijls, N.H., et al., Fractional flow reserve: a useful index to evaluate the influence of an epicardial coronary stenosis on myocardial blood flow. Circulation, 1995. 92(11): p. 3183-3193.

39. Wilson, R.F., et al., Effects of adenosine on human coronary arterial circulation. Circulation, 1990. 82(5): p. 1595-1606.

40. Rajiah, P. and C.D. Maroules, Myocardial ischemia testing with computed tomography: emerging strategies. Cardiovascular diagnosis and therapy, 2017. 7(5): p. 475. 
41. Abbara, S., et al., SCCT guidelines for the performance and acquisition of coronary computed tomographic angiography: A report of the society of Cardiovascular Computed Tomography Guidelines Committee: Endorsed by the North American Society for Cardiovascular Imaging (NASCI). Journal of cardiovascular computed tomography, 2016. 10(6): p. 435-449.

42. Nørgaard, B.L., et al., Diagnostic performance of noninvasive fractional flow reserve derived from coronary computed tomography angiography in suspected coronary artery disease: the NXT trial (Analysis of Coronary Blood Flow Using CT Angiography: Next Steps). Journal of the American College of Cardiology, 2014. 63(12): p. 1145-1155.

43. Min, J.K., et al., Diagnostic accuracy of fractional flow reserve from anatomic CT angiography. Jama, 2012. 308(12): p. 1237-1245.

44. Koo, B.-K., et al., Diagnosis of ischemia-causing coronary stenoses by noninvasive fractional flow reserve computed from coronary computed tomographic angiograms: results from the prospective multicenter DISCOVER-FLOW (Diagnosis of IschemiaCausing Stenoses Obtained Via Noninvasive Fractional Flow Reserve) study. Journal of the American College of Cardiology, 2011. 58(19): p. 1989-1997.

45. Gaur, S., et al., Rationale and design of the HeartFlowNXT (HeartFlow analysis of coronary blood flow using CT angiography: NeXt sTeps) study. Journal of cardiovascular computed tomography, 2013. 7(5): p. 279-288.

46. Modi, B.N., et al., Predicting the Physiological Effect of Revascularization in Serially Diseased Coronary Arteries: Clinical Validation of a Novel CT Coronary 
Angiography-Based Technique. Circulation: Cardiovascular Interventions, 2019. 12(2): p. e007577.

47. Zhong, L., et al., Application of patient-specific computational fluid dynamics in coronary and intra-cardiac flow simulations: Challenges and opportunities. Frontiers in physiology, 2018. 9.

48. Tröbs, M., et al., Comparison of fractional flow reserve based on computational fluid dynamics modeling using coronary angiographic vessel morphology versus invasively measured fractional flow reserve. The American journal of cardiology, 2016. 117(1): p. 29-35.

49. Tu, S., et al., Fractional flow reserve calculation from 3-dimensional quantitative coronary angiography and TIMI frame count: a fast computer model to quantify the functional significance of moderately obstructed coronary arteries. JACC: Cardiovascular Interventions, 2014. 7(7): p. 768-777.

50. Bluestein, D., et al., Vortex shedding in steady flow through a model of an arterial stenosis and its relevance to mural platelet deposition. Annals of biomedical engineering, 1999. 27(6): p. 763-773.

51. Cao, J. and S.E. Rittgers, Particle motion within in vitro models of stenosed internal carotid and left anterior descending coronary arteries. Annals of biomedical engineering, 1998. 26(2): p. 190-199.

52. Stroud, J., S. Berger, and D. Saloner, Influence of stenosis morphology on flow through severely stenotic vessels: implications for plaque rupture. Journal of biomechanics, 2000. 33(4): p. 443-455. 
53. Morris, P.D., et al., Virtual fractional flow reserve from coronary angiography: modeling the significance of coronary lesions: results from the VIRTU-1 (VIRTUal Fractional Flow Reserve From Coronary Angiography) study. JACC: Cardiovascular Interventions, 2013. 6(2): p. 149-157.

54. Tu, S., et al., Diagnostic accuracy of fast computational approaches to derive fractional flow reserve from diagnostic coronary angiography: the international multicenter FAVOR pilot study. JACC: cardiovascular interventions, 2016. 9(19): p. 2024-2035.

55. Pellicano, M., et al., Validation study of image-based fractional flow reserve during coronary angiography. Circulation: Cardiovascular Interventions, 2017. 10(9): p. e005259.

56. Fogler, H.S., Essentials of Chemical Reaction Engineering: Essenti Chemica Reactio Engi. 2010: Pearson Education.

57. Mac Mullin, R., The Theory of Short Circuiting in Continuous-Flow Mixing Vessels in Series and the Kinetics of Chemical Reactions in Such Systems. Trans, Amer. Inst. Chem. Engrs, 1935. 31: p. 409-458.

58. Dankwerts, P., Continuous flow systems. Distribution of residence times. Chem. Eng. Sci, 1953. 2(1): p. 1-13.

59. Danckwerts, P., The effect of incomplete mixing on homogeneous reactions. Chemical Engineering Science, 1958. 8(1-2): p. 93-102.

60. Sandberg, M., What is ventilation efficiency? Building and environment, 1981. 16(2): p. 123-135. 
61. Spalding, D., A note on mean residence-times in steady flows of arbitrary complexity. Chemical Engineering Science, 1958. 9(1): p. 74-77.

62. Baléo, J.N. and P. Le Cloirec, Validating a prediction method of mean residence time spatial distributions. AIChE journal, 2000. 46(4): p. 675-683.

63. Liu, M. and J. Tilton, Spatial distributions of mean age and higher moments in steady continuous flows. AIChE journal, 2010. 56(10): p. 2561-2572.

64. Liu, M., Age distribution and the degree of mixing in continuous flow stirred tank reactors. Chemical Engineering Science, 2012. 69(1): p. 382-393.

65. Zwietering, T.N., Suspending of solid particles in liquid by agitators. Chemical Engineering Science, 1958. 8(3-4): p. 244-253.

66. Russ, D.C. and R.E. Berson, Mean age theory in multiphase systems. Chemical Engineering Science, 2016. 141: p. 1-7.

67. Russ, D.C. and R.E. Berson, Computationally determined just suspended speed using multiphase mean age theory. Chemical Engineering Research and Design, 2016. 114: p. 13-17.

68. Kunov, M.J., D. Steinman, and C.R. Ethier, Particle volumetric residence time calculations in arterial geometries. Journal of biomechanical engineering, 1996. 118(2): p. 158-164.

69. König, C. and C. Clark, Flow mixing and fluid residence times in a model of a ventricular assist device. Medical engineering \& physics, 2001. 23(2): p. 99-110.

70. Ford, M.D., et al., Virtual angiography for visualization and validation of computational models of aneurysm hemodynamics. IEEE transactions on medical imaging, 2005. 24(12): p. 1586-1592. 
71. Bernsdorf, J., et al., Applying the lattice Boltzmann technique to biofluids: A novel approach to simulate blood coagulation. Computers \& Mathematics with Applications, 2008. 55(7): p. 1408-1414.

72. Gay, M. and L.T. Zhang, Numerical studies of blood flow in healthy, stenosed, and stented carotid arteries. International journal for numerical methods in fluids, 2009. 61(4): p. 453-472.

73. Rayz, V., et al., Flow residence time and regions of intraluminal thrombus deposition in intracranial aneurysms. Annals of biomedical engineering, 2010. 38(10): p. 30583069.

74. Suh, G.-Y., et al., Quantification of particle residence time in abdominal aortic aneurysms using magnetic resonance imaging and computational fluid dynamics. Annals of biomedical engineering, 2011. 39(2): p. 864-883.

75. Long, C., et al., Computation of residence time in the simulation of pulsatile ventricular assist devices. Computational Mechanics, 2014. 54(4): p. 911-919.

76. Arzani, A., Accounting for residence-time in blood rheology models: do we really need non-Newtonian blood flow modelling in large arteries? Journal of The Royal Society Interface, 2018. 15(146): p. 20180486.

77. Reza, M.M.S. and A. Arzani, A critical comparison of different residence time measures in aneurysms. Journal of biomechanics, 2019. 88: p. 122-129.

78. Himburg, H.A., et al., Spatial comparison between wall shear stress measures and porcine arterial endothelial permeability. American Journal of Physiology-Heart and Circulatory Physiology, 2004. 286(5): p. H1916-H1922. 
79. Dong, J., K.K. Wong, and J. Tu, Hemodynamics analysis of patient-specific carotid bifurcation: A CFD model of downstream peripheral vascular impedance. International journal for numerical methods in biomedical engineering, 2013. 29(4): p. 476-491.

80. Arzani, A., et al., Wall shear stress exposure time: a Lagrangian measure of near-wall stagnation and concentration in cardiovascular flows. Biomechanics and modeling in mechanobiology, 2017. 16(3): p. 787-803.

81. Malota, Z., et al., Numerical analysis of the impact of flow rate, heart rate, vessel geometry, and degree of stenosis on coronary hemodynamic indices. BMC cardiovascular disorders, 2018. 18(1): p. 132.

82. Jung, J., A. Hassanein, and R.W. Lyczkowski, Hemodynamic computation using multiphase flow dynamics in a right coronary artery. Annals of biomedical engineering, 2006. 34(3): p. 393.

83. Baieth, H.A., Physical parameters of blood as a non-Newtonian fluid. International journal of biomedical science: IJBS, 2008. 4(4): p. 323.

84. Sakariassen, K.S., L. Orning, and V.T. Turitto, The impact of blood shear rate on arterial thrombus formation. Future science OA, 2015. 1(4).

85. Davies, J., et al. Evidence of a dominant backward-propagating. 2006. BMJ PUBLISHING GROUP.

86. Frigui, H., L. Zhang, and P.D. Gader, Context-dependent multisensor fusion and its application to land mine detection. IEEE Transactions on Geoscience and Remote Sensing, 2010. 48(6): p. 2528-2543. 
87. Abdallah, A.C.B., H. Frigui, and P. Gader, Adaptive local fusion with fuzzy integrals. IEEE Transactions on fuzzy systems, 2012. 20(5): p. 849-864.

88. Tibshirani, R., Regression shrinkage and selection via the lasso. Journal of the Royal Statistical Society: Series B (Methodological), 1996. 58(1): p. 267-288.

89. Chun, H. and S. Keleş, Sparse partial least squares regression for simultaneous dimension reduction and variable selection. Journal of the Royal Statistical Society: Series B (Statistical Methodology), 2010. 72(1): p. 3-25.

90. Govaert, G. and M. Nadif, Co-clustering: models, algorithms and applications. 2013: John Wiley \& Sons.

91. Bartúnek, J., et al., Quantitative coronary angiography in predicting functional significance of stenoses in an unselected patient cohort. Journal of the American College of Cardiology, 1995. 26(2): p. 328-334.

92. Vardhan, M., et al., The importance of side branches in modeling 3D hemodynamics from angiograms for patients with coronary artery disease. Scientific Reports, 2019. 9(1): p. 8854.

93. Markl, M., P.J. Kilner, and T. Ebbers, Comprehensive $4 D$ velocity mapping of the heart and great vessels by cardiovascular magnetic resonance. Journal of Cardiovascular Magnetic Resonance, 2011. 13(1): p. 7.

94. Hong, H., et al., Characteristics of pulsatile flows in curved stenosed channels. PloS one, 2017. 12(10): p. e0186300.

95. Ha, H., et al., Estimating the irreversible pressure drop across a stenosis by quantifying turbulence production using 4D Flow MRI. Scientific reports, 2017. 7: p. 46618. 
96. Biasetti, J., et al., Synergy between shear-induced migration and secondary flows on red blood cells transport in arteries: considerations on oxygen transport. Journal of the Royal Society Interface, 2014. 11(97): p. 20140403.

97. Guerciotti, B., et al., Computational study of the risk of restenosis in coronary bypasses. Biomechanics and modeling in mechanobiology, 2017. 16(1): p. 313-332. 


\section{CURRICULUM VITA}

NAME: Javad Hashemi

DOB: Zabol, Iran - September 08, 1987

\begin{tabular}{l|c|c|c}
\hline \multicolumn{1}{c|}{ Institution and Location } & Degree & $\begin{array}{c}\text { Completion Date } \\
\text { MM/YYYY }\end{array}$ & Field of Study \\
\hline $\begin{array}{l}\text { University of Sistan and } \\
\text { Baluchestan } \\
\text { Tarbiat Modares University }\end{array}$ & $\mathrm{BS}$ & $09 / 2010$ & Chemical Engineering \\
University of Louisville,KY & $\mathrm{PhD}$ & $12 / 2019$ & $\begin{array}{l}\text { Chemical Engineering- } \\
\text { Chemical }\end{array}$ \\
\hline
\end{tabular}

Publications

1. J. Hashemi, S. Rai, N. S. V. Singam, S. Ghafghazi, R. Eric Berson. Blood Residence Time to Assess Significance of Coronary Artery Stenosis. Scientific Reports, Under Review.

2. J. Hashemi, S. Ghafghazi, R. Eric Berson. Diagnosing serial coronary artery stenoses using blood residence time. Ready to Submit.

3. J. Hashemi, S. Ghafghazi, R. Eric Berson. Non-invasive diagnosis of coronary artery stenosis in real time. Ready to Submit.

4. M. Hashemi, A. Zali, J. Hashemi, S. Oraee-Yazdani, A. Akbari. Down-regulation of 14-3-3 zeta sensitizes human glioblastoma cells to apoptosis induction. Apoptosis. 2018, 23(11-12):616-625.

5. J. Hashemi, S. Hashemi-Najafabadi, E. Vasheghani-Farahani. Synergistic effect of PEGylation and pentoxifylline addition on immunoprotection of pancreatic islets, Journal of Biomaterials Science, Polymer Edition, 2017; 28:33-49.

6. F. Bahrami, M. Hashemi, F. Khalili, J. Hashemi, A. Asgari. Stimulation of cannabinoid CB1 and n-methyl-d-aspartate receptors increases neuroprotective effect against diazinoninduced neurotoxicity, Neurophysiology, 2013; 45(5-6): 433-440. 
7. J. Hashemi, A. Samimi. Steady state electric power generation in up-flow microbial fuel cell using the estimated time span method for bacteria growth in domestic wastewater, Biomass and Bioenergy, 2012; 45: 65-76.

Presentations

1. J. Hashemi, N. S. Singam, T. R. Huffman, R. E. Berson, S. Ghafghazi. Virtual fractional flow reserve computed through design of experiments has significant correlation with pressure wire fractional flow reserve. 2019 the American Heart Association's Scientific Sessions, Philadelphia, PA, November 16-18.

2. J. Hashemi, S. Rai, S. Ghafghazi, R. E. Berson, Non-Invasive assessment of coronary stenosis w/ mean age theory. 2019 IEEE Engineering in Medicine \& Biology Society (EMBC), Berlin, Germany, July 23-27.

3. J. Hashemi, R.E. Berson, S. Ghafghazi. Novel non-invasive quantification of coronary artery stenosis. 2018 BMES Annual Meeting, Atlanta, GA, October 17-20, 2018.

4. J. Hashemi, S. Ghafghazi, R. E. Berson. Effect of Physiological and Anatomical Parameters on Fractional Flow Reserve (FFR) of Coronary Artery Stenosis. 2017 AIChE Annual Meeting, Minnesota, MN, October 29-November 3.

Patents

1. J. Hashemi, R. E. Berson, S. Ghafghazi. System and Method for Determining Fractional Flow Reserve Through a Coronary Stenosis. U.S. Patent Application 62803636, filed February 2019. Provisional Patent.

2. R. E. Berson, J. Hashemi, S. Ghafghazi. Method and System for Assessing a Coronary Stenosis. U.S. Patent Application 62701136, filed July 2018. Provisional Patent.

Awards and Honor

2018 CO-PI, EXCITE Fund (NIH REACH), Non-Invasive Real-Time Assessment of Coronary Stenosis Using RBC AGE, $\$ 200,000$

2018 Entrepreneurial Lead, NSF Innovation Corps (I-Corps) \$2500

2014 University of Louisville, University Fellowship, $(\$ 93,876.00$ for two years). 